\title{
Bufadienolides from the eggs of toad Bufo bufo gargarizans and their anti-melanoma activities
}

Shi-wen Zhou, ${ }^{\dagger}$, ,"Jing-yu Quan, ${ }^{+,}, "$ Zi-wei Li, ${ }^{\dagger}$ Ge Ye, ${ }^{\dagger}$ Zhuo Shang, ${ }^{\S}$ Ze-ping Chen, ${ }^{\dagger}$ Lei

Wang, ${ }^{\dagger}$ Xin-yuan Li, ${ }^{\dagger}$ Xiao-qi Zhang, ${ }^{\dagger}$ Jie Li, ${ }^{*}{ }^{\S}$ Jun-shan Liu, ${ }^{*},+$ and Hai-yan

$$
\operatorname{Tian}^{*}+, \S
$$

${ }^{\dagger}$ Institute of Traditional Chinese Medicine and Natural Products, College of

Pharmacy, Jinan University, Guangzhou 510632, People’s Republic of China

$\$$ Guangdong Provincial Key Laboratory of Chinese Medicine Pharmaceutics, School

of Traditional Chinese Medicine, Southern Medical University, Guangzhou, 510515,

People's Republic of China

${ }^{\S}$ Department of Chemistry and Biochemistry, University of South Carolina, Columbia 29208, US

${ }^{\perp}$ Department of Biomedical Sciences, City University of Hong Kong, Hong Kong, SAR 999077, People’s Republic of China 


\section{Supporting Information Contents}

\section{Additional Crystallographic Data for Compound 2 .....................................1}

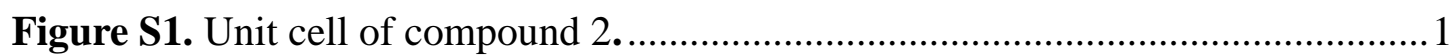

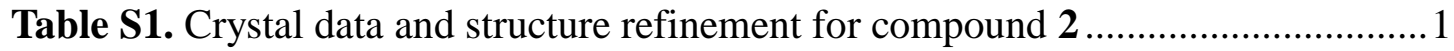

Table S2. Fractional Atomic Coordinates $\left(\times 10^{4}\right)$ and Equivalent Isotropic

Displacement Parameters $\left(\AA^{2} \times 10^{3}\right)$ for compound 2. $U_{\text {eq }}$ is defined as $1 / 3$ of of the trace

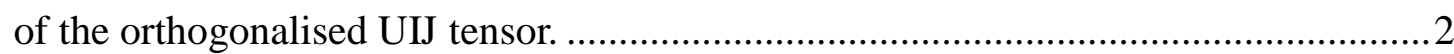

Table S3. Anisotropic Displacement Parameters $\left(\AA 2 \times 10^{3}\right)$ for compound 2...............4

Table S4. Bond Lengths for compound 2 ........................................................

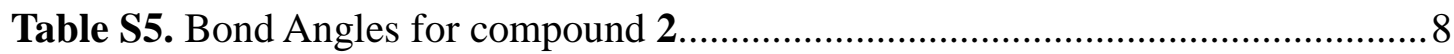

Table S6. Hydrogen Atom Coordinates $\left(\AA \times 10^{4}\right)$ and Isotropic Displacement

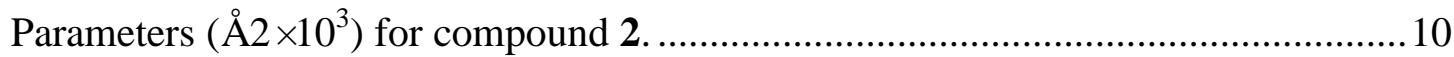

2. Spectra of Compounds 1-3

Figure S2. The UV spectrum of compound 1 in $\mathrm{MeOH}$........................................... 14

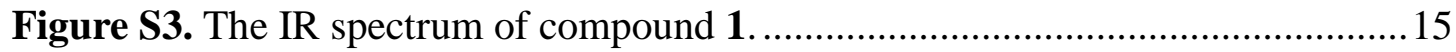

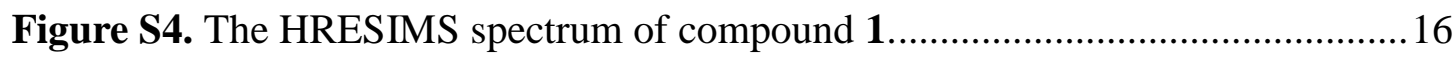

Figure S5. The ${ }^{1} \mathrm{H}-\mathrm{NMR}$ spectrum of compound 1 (in $\mathrm{CD}_{3} \mathrm{OD}, 500 \mathrm{MHz}$ )...............17

Figure S6. The ${ }^{13} \mathrm{C}$-NMR spectrum of compound 1 (in $\mathrm{CD}_{3} \mathrm{OD}, 125 \mathrm{MHz}$ ).............18

Figure S7. The ${ }^{13} \mathrm{C}-\mathrm{NMR}$ and DEPT 135 spectra of compound $\mathbf{1}$ (in $\mathrm{CD}_{3} \mathrm{OD}$ )........19

Figure S8. The ${ }^{1} \mathrm{H}-{ }^{1} \mathrm{H}$ COSY spectrum of compound $\mathbf{1}$ (in $\mathrm{CD}_{3} \mathrm{OD}$ ) .......................20

Figure S9. The HSQC spectrum of compound 1 (in $\mathrm{CD}_{3} \mathrm{OD}$ ) ................................21

Figure S10. The HMBC spectrum of compound 1 (in $\mathrm{CD}_{3} \mathrm{OD}$ ) ............................22

Figure S11. The NOESY spectrum of Compound 1 (in $\mathrm{CD}_{3} \mathrm{OD}$ )............................23

Figure S12. The UV spectrum of compound 2 (in $\mathrm{MeOH}$ ). ...................................2.

Figure S13. The IR spectrum of compound 2 (in $\mathrm{KBr}$ )......................................25

Figure S16. The ${ }^{13} \mathrm{C}-\mathrm{NMR}$ spectrum of compound 2 (in $\mathrm{CD}_{3} \mathrm{OD}, 125 \mathrm{MHz}$ ). ..........28

Figure S17. The ${ }^{13} \mathrm{C}-\mathrm{NMR}$ and DEPT 135 spectra of compound 2 (in $\mathrm{CD}_{3} \mathrm{OD}$ )......29 
Figure S18. The ${ }^{1} \mathrm{H}-{ }^{1} \mathrm{H}$ COSY spectrum of compound 2 (in $\mathrm{CD}_{3} \mathrm{OD}$ ) .......................30

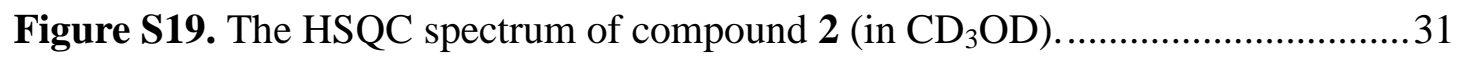

Figure S20. The HMBC spectrum of compound 2 (in $\mathrm{CD}_{3} \mathrm{OD}$ ) .................................

Figure S21. The NOESY spectrum of compound 2 (in CD3OD)..............................33

Figure S22. The UV spectrum of compound 3 (in $\mathrm{MeOH}$ ). ..................................... 34

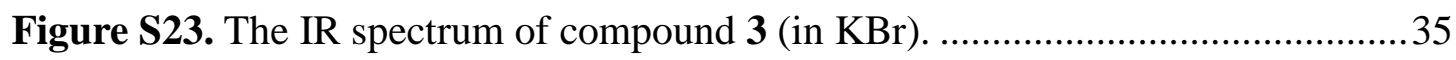

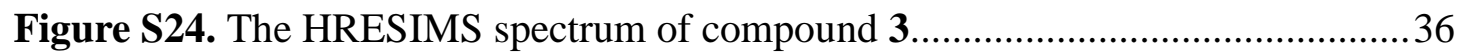

Figure S26. The ${ }^{13} \mathrm{C}-\mathrm{NMR}$ spectrum of compound 3 (in $\mathrm{CD}_{3} \mathrm{OD}, 100 \mathrm{MHz}$ ). ...........38

Figure S27. The ${ }^{13} \mathrm{C}-\mathrm{NMR}$ and DEPT 135 spectra of compound $\mathbf{3}$ (in $\mathrm{CD}_{3} \mathrm{OD}$ ).......39

Figure S28. The ${ }^{1} \mathrm{H}-{ }^{1} \mathrm{H}$ COSY spectrum of compound $\mathbf{3}$ (in $\mathrm{CD}_{3} \mathrm{OD}$ ) .....................40

Figure S29. The HSQC spectrum of compound 3 (in $\mathrm{CD}_{3} \mathrm{OD}$ ) ..............................41

Figure S30. The HMBC spectrum of compound 3 (in $\mathrm{CD}_{3} \mathrm{OD}$ ) .............................42

Figure S31. The NOESY spectrum of compound 3 (in $\mathrm{CD}_{3} \mathrm{OD}$ )...........................43

Figure S32. Enlarged ${ }^{1} \mathrm{H}$ NMR spectrum of (S)-MTPA-ester of compound 3 ...........44

Figure S33. Chiral HPLC analysis result of compound 3 .....................................45

Figure S34. The ${ }^{1} \mathrm{H}-\mathrm{NMR}$ spectrum of bufalin (in $\mathrm{CD}_{3} \mathrm{OD}, 600 \mathrm{MHz}$ ) ...................46

Figure S35. The ${ }^{13} \mathrm{C}-\mathrm{NMR}$ spectrum of bufalin (in $\mathrm{CD}_{3} \mathrm{OD}, 400 \mathrm{MHz}$ ) ..................47

Figure S36. The ${ }^{13} \mathrm{C}-\mathrm{NMR}$ and DEPT 135 spectra of bufalin (in $\mathrm{CD}_{3} \mathrm{OD}$ ) ...............48

Figure S37. The ${ }^{1} \mathrm{H}^{1}{ }^{\mathrm{H}} \mathrm{COSY}$ spectrum of bufalin (in CD3OD) .............................49

Figure S38. The HSQC spectrum of bufalin (in $\mathrm{CD}_{3} \mathrm{OD}$ ). ......................................50

Figure S39. The HMBC spectrum of bufalin (in $\mathrm{CD}_{3} \mathrm{OD}$ ) ...................................51

Figure S40. The ${ }^{1} \mathrm{H}-\mathrm{NMR}$ spectrum of hellebrigenin (6) (in $\mathrm{CD}_{3} \mathrm{OD}, 400 \mathrm{MHz}$ )......52

Figure S41. The ${ }^{13} \mathrm{C}-\mathrm{NMR}$ spectrum of hellebrigenin (6) (in $\mathrm{CD}_{3} \mathrm{OD}, 400 \mathrm{MHz}$ ).....53

Figure S42. The ${ }^{13} \mathrm{C}-\mathrm{NMR}$ and DEPT 135 spectra of hellebrigenin (6) (in $\mathrm{CD}_{3} \mathrm{OD}$ ). 54

Figure S43. The ${ }^{1} \mathrm{H}-\mathrm{NMR}$ spectrum of 4 (in $\mathrm{CD}_{3} \mathrm{OD}, 400 \mathrm{MHz}$ )............................55

Figure S44. The ${ }^{13} \mathrm{C}$-NMR spectrum of 4 (in $\mathrm{CD}_{3} \mathrm{OD}, 400 \mathrm{MHz}$ ) ............................56

Figure S45. The ${ }^{13} \mathrm{C}-\mathrm{NMR}$ and DEPT 135 spectra of $\mathbf{4}$ (in $\mathrm{CD}_{3} \mathrm{OD}, 400 \mathrm{MHz}$ ) ........57

Figure S46. The ${ }^{1} \mathrm{H}-\mathrm{NMR}$ spectrum of $\mathbf{5}$ (in $\mathrm{CD}_{3} \mathrm{OD}, 400 \mathrm{MHz}$ )..............................58

Figure S47. The ${ }^{13} \mathrm{C}-\mathrm{NMR}$ spectrum of $\mathbf{5}$ (in $\mathrm{CD}_{3} \mathrm{OD}, 400 \mathrm{MHz}$ ) ...........................59

Figure S48. The ${ }^{13} \mathrm{C}-\mathrm{NMR}$ and DEPT 135 spectra of 5 (in $\mathrm{CD}_{3} \mathrm{OD}, 400 \mathrm{MHz}$ ) ........60 
Figure S49. The ${ }^{1} \mathrm{H}-\mathrm{NMR}$ spectrum of 7 (in $\mathrm{CD}_{3} \mathrm{OD}, 400 \mathrm{MHz}$ ) ............................6.

Figure S50. The ${ }^{13} \mathrm{C}-\mathrm{NMR}$ spectrum of 7 (in $\mathrm{CD}_{3} \mathrm{OD}, 400 \mathrm{MHz}$ ) ..........................62

Figure S51. The ${ }^{13} \mathrm{C}-\mathrm{NMR}$ and DEPT 135 spectra of 7 (in $\mathrm{CD}_{3} \mathrm{OD}, 400 \mathrm{MHz}$ ) ........63

Figure S52. The ${ }^{1} \mathrm{H}-\mathrm{NMR}$ spectrum of $\mathbf{8}$ (in $\mathrm{CD}_{3} \mathrm{OD}, 400 \mathrm{MHz}$ ) .............................64

Figure S53. The ${ }^{13} \mathrm{C}-\mathrm{NMR}$ spectrum of $\mathbf{8}$ (in $\mathrm{CD}_{3} \mathrm{OD}, 400 \mathrm{MHz}$ ) ..........................6...6

Figure S54. The ${ }^{13} \mathrm{C}-\mathrm{NMR}$ and DEPT 135 spectra of 8 (in $\mathrm{CD}_{3} \mathrm{OD}, 400 \mathrm{MHz}$ ) ........66

Figure S55. The ${ }^{1} \mathrm{H}-\mathrm{NMR}$ spectrum of 9 (in $\mathrm{CD}_{3} \mathrm{OD}, 400 \mathrm{MHz}$ ) ............................67

Figure S56. The ${ }^{13} \mathrm{C}-\mathrm{NMR}$ spectrum of 9 (in $\mathrm{CD}_{3} \mathrm{OD}, 400 \mathrm{MHz}$ ) ...........................6...6

Figure S57. The ${ }^{13} \mathrm{C}-\mathrm{NMR}$ and DEPT 135 spectra of 9 (in $\mathrm{CD}_{3} \mathrm{OD}, 400 \mathrm{MHz}$ )........69

3. Identification of Compounds 1-3 in the total ethanol extract of toad eggs....70

Figure S58. Identification of compound 1 in total ethanol extract of toad eggs using HPLC-TOF-MS [retention time: $58.765 \mathrm{~min}, \mathrm{~m} / z 367.1880[\mathrm{M}+\mathrm{H}]^{+}\left(\right.$calcd for $\mathrm{C}_{23} \mathrm{H}_{27} \mathrm{O}_{4}$,

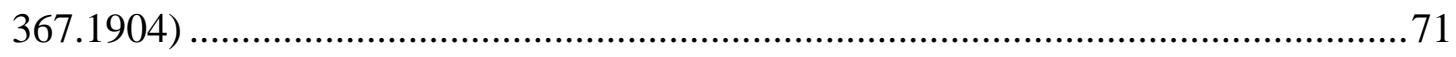

Figure S59. Identification of compound $\mathbf{3}$ in total ethanol extract of toad eggs using HPLC-TOF-MS [retention time: $37.259 \mathrm{~min}, \mathrm{~m} / z$ $433.2230[\mathrm{M}+\mathrm{H}]^{+}\left(\right.$calcd for $\mathrm{C}_{24} \mathrm{H}_{33} \mathrm{O}_{7}$, 433.2221)

Figure S60. Identification of compound $\mathbf{3}$ in total ethanol extract of toad eggs using HPLC-TOF-MS [retention time: $40.929 \mathrm{~min}, \mathrm{~m} / z 489.2488[\mathrm{M}+\mathrm{H}]^{+}\left(\right.$calcd for $\mathrm{C}_{27} \mathrm{H}_{37} \mathrm{O}_{8}$, 489.2483) .73

4. Comparison NMR data of 1 and 2 with those of known compounds..............74

Table S7. ${ }^{1} \mathrm{H}$ and ${ }^{13} \mathrm{C}$ NMR data of compound $\mathbf{1}$ and bufogargarizin $\mathrm{C}$......................74

Table S8 ${ }^{1} \mathrm{H}$ and ${ }^{13} \mathrm{C}$ NMR data of compound 2 and hellebrigenin.......................... 75

5. Proposed biosynthesis pathway of 1 ...........................................................75

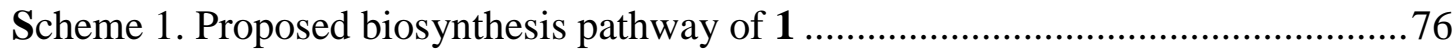




\section{Additional Crystallographic Data for Compound 2}

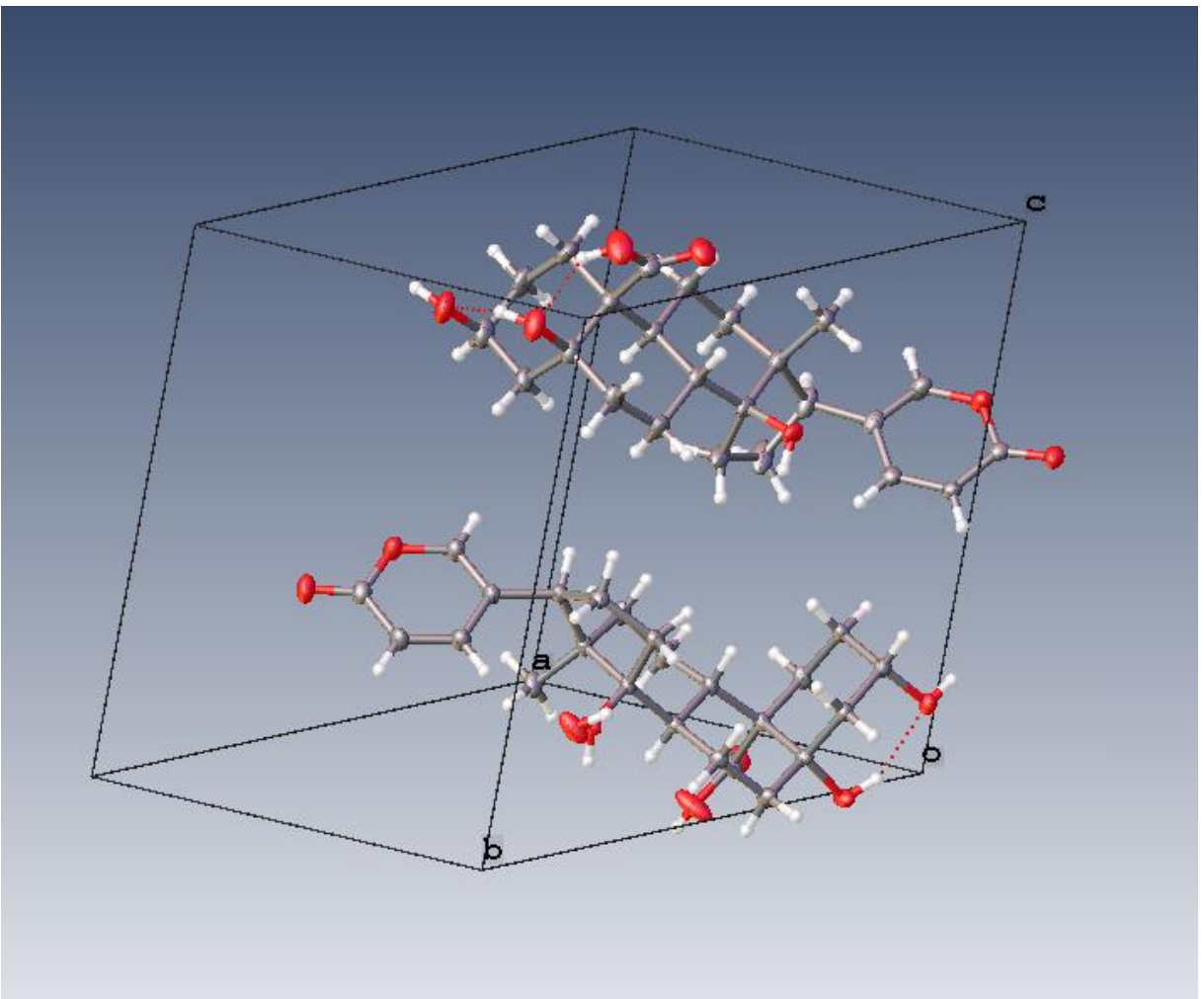

Figure S1. Unit cell of compound 2.

Table S1. Crystal data and structure refinement for compound 2

\begin{tabular}{|c|c|}
\hline Identification code & Compound 2 \\
\hline Empirical formula & $\mathrm{C}_{48} \mathrm{H}_{66} \mathrm{O}_{15}$ \\
\hline Formula weight & 883.00 \\
\hline Temperature/K & $100.00(10)$ \\
\hline Crystal system & monoclinic \\
\hline Space group & $\mathrm{P} 2_{1}$ \\
\hline $\mathrm{a} / \AA ̊$ & $13.7133(2)$ \\
\hline $\mathrm{b} / \AA$ & $12.14470(10)$ \\
\hline $\mathrm{c} / \AA$ & $13.78260(10)$ \\
\hline$\alpha /^{\circ}$ & 90 \\
\hline$\beta /^{\circ}$ & $109.4670(10)$ \\
\hline$\gamma /^{\circ}$ & 90 \\
\hline Volume $/ \AA^{3}$ & 2164.19(4) \\
\hline
\end{tabular}




\begin{tabular}{|l|l|}
\hline $\mathrm{Z}$ & 2 \\
\hline$\rho_{\text {calc }} \mathrm{g} / \mathrm{cm}^{3}$ & 1.355 \\
\hline$\mu / \mathrm{mm}^{-1}$ & 0.824 \\
\hline $\mathrm{F}(000)$ & 948.0 \\
\hline Crystal size/mm ${ }^{3}$ & $0.40 \times 0.26 \times 0.20$ \\
\hline Radiation & $\mathrm{Cu} \mathrm{K} \alpha(\lambda=1.54178)$ \\
\hline $2 \theta \quad$ range for data collection/ ${ }^{\circ}$ & 6.802 to 147.376 \\
\hline Index ranges & $-17 \leq \mathrm{h} \leq 16,-14 \leq \mathrm{k} \leq 15,-17 \leq 1 \leq 17$ \\
\hline Reflections collected & 41331 \\
\hline Independent reflections & $8609\left[R_{\text {int }}=0.0390, R_{\text {sigma }}=0.0257\right]$ \\
\hline Data/restraints/parameters & $8609 / 1 / 581$ \\
\hline Goodness-of-fit on $\mathrm{F}^{2}$ & 0.998 \\
\hline Final R indexes [I $>=2 \sigma(\mathrm{I})]$ & $R_{1}=0.0354, w R_{2}=0.1134$ \\
\hline Final R indexes [all data] & $R_{1}=0.0364, w R_{2}=0.1151$ \\
\hline Largest diff. peak/hole / e $\AA^{-3}$ & $0.38 /-0.33$ \\
\hline Flack parameter & $0.10(5)$ \\
\hline
\end{tabular}

Table S2. Fractional Atomic Coordinates $\left(\times 10^{4}\right)$ and Equivalent Isotropic Displacement Parameters $\left(\AA^{2} \times 10^{3}\right)$ for compound 2. $U_{\text {eq }}$ is defined as $1 / 3$ of of the trace of the orthogonalised UIJ tensor.

\begin{tabular}{|l|l|l|l|l|}
\hline Atom & \multicolumn{1}{|c|}{$\boldsymbol{x}$} & \multicolumn{1}{c|}{$\boldsymbol{y}$} & \multicolumn{1}{c|}{$\boldsymbol{z}$} & $\mathbf{U}(\mathbf{e q})$ \\
\hline O1W & $-412.3(17)$ & $8870.6(16)$ & $2446.5(17)$ & $36.4(4)$ \\
\hline O1 & $5829.5(17)$ & $7748.6(18)$ & $8815.3(17)$ & $41.1(5)$ \\
\hline O2 & $3898.3(16)$ & $7403.1(17)$ & $8760.8(16)$ & $36.0(4)$ \\
\hline O3 & $3462(2)$ & $6156(2)$ & $10021.4(19)$ & $49.6(6)$ \\
\hline O4 & $3209.3(18)$ & $4405.8(19)$ & $9666.8(15)$ & $40.5(5)$ \\
\hline O5 & $1606.8(12)$ & $3011.5(15)$ & $6413.3(12)$ & $24.2(3)$ \\
\hline O6 & $1492.3(14)$ & $-1188.9(15)$ & $6271.9(13)$ & $29.4(4)$ \\
\hline O7 & $-169.3(15)$ & $-1564.3(16)$ & $5480.1(13)$ & $30.5(4)$ \\
\hline
\end{tabular}




\begin{tabular}{|l|l|l|l|l|}
\hline C1 & $5378(2)$ & $5528(2)$ & $9492.8(19)$ & $33.7(6)$ \\
\hline C2 & $6092(2)$ & $5761(3)$ & $8870(2)$ & $36.8(6)$ \\
\hline C3 & $5742(2)$ & $6777(2)$ & $8192(2)$ & $34.2(6)$ \\
\hline C4 & $4614(2)$ & $6699(2)$ & $7518.3(19)$ & $28.5(5)$ \\
\hline C5 & $3890.8(19)$ & $6443(2)$ & $8119.2(19)$ & $26.3(5)$ \\
\hline C6 & $2766(2)$ & $6311(2)$ & $7425(2)$ & $32.3(5)$ \\
\hline C7 & $2582.5(19)$ & $5271(2)$ & $6763(2)$ & $30.4(5)$ \\
\hline C8 & $2950.5(17)$ & $4230.0(19)$ & $7411.0(16)$ & $20.5(4)$ \\
\hline C9 & $4098.9(18)$ & $4363(2)$ & $8070.2(16)$ & $22.2(4)$ \\
\hline C10 & $4239.4(19)$ & $5387(2)$ & $8794.8(17)$ & $24.5(5)$ \\
\hline C11 & $4575.7(18)$ & $3300(2)$ & $8623.5(19)$ & $27.5(5)$ \\
\hline C12 & $4376.7(19)$ & $2343(2)$ & $7853(2)$ & $29.7(5)$ \\
\hline C13 & $3218.0(18)$ & $2127(2)$ & $7289.3(18)$ & $24.2(5)$ \\
\hline C14 & $2714.2(17)$ & $3185(2)$ & $6731.4(16)$ & $21.3(4)$ \\
\hline C15 & $3059(2)$ & $3234(2)$ & $5781.5(19)$ & $32.2(6)$ \\
\hline C16 & $3270(3)$ & $2032(3)$ & $5526(2)$ & $44.9(8)$ \\
\hline C17 & $3172(2)$ & $1294(2)$ & $6398(2)$ & $32.0(6)$ \\
\hline C18 & $2708.8(19)$ & $1692(2)$ & $8045.7(18)$ & $26.6(5)$ \\
\hline C19 & $3582(2)$ & $5258(2)$ & $9517.3(19)$ & $33.1(6)$ \\
\hline C20 & $2242.7(18)$ & $537(2)$ & $6071.5(17)$ & $24.5(5)$ \\
\hline C21 & $2305(2)$ & $-471(2)$ & $6496.7(19)$ & $29.5(5)$ \\
\hline C22 & $1274.3(19)$ & $817(2)$ & $5313.4(16)$ & $23.2(4)$ \\
\hline C23 & $461.0(17)$ & $111.0(19)$ & $5070.8(15)$ & $21.1(4)$ \\
\hline C24 & $535.2(18)$ & $-917.0(19)$ & $5581.4(15)$ & $22.4(4)$ \\
\hline O6A & $4648.3(13)$ & $9047.0(14)$ & $4869.5(13)$ & $26.1(4)$ \\
\hline O7A & $4831.0(15)$ & $10754.1(16)$ & $4412.1(15)$ & $32.4(4)$ \\
\hline O5A & $813.9(13)$ & $7260.7(12)$ & $1998.5(11)$ & $18.6(3)$ \\
\hline O4A & $1170(2)$ & $4137.1(18)$ & $-60.9(17)$ & $48.3(6)$ \\
\hline O3A & $1972.1(15)$ & $2548.4(16)$ & $373.4(14)$ & $31.6(4)$ \\
\hline
\end{tabular}




\begin{tabular}{|l|l|l|l|l|}
\hline O1A & $-762.2(13)$ & $913.9(13)$ & $1550.9(12)$ & $23.1(3)$ \\
\hline O2A & $-667.8(14)$ & $2383.3(13)$ & $78.0(11)$ & $23.0(3)$ \\
\hline C1A & $1255.4(18)$ & $2049.2(17)$ & $1878.8(18)$ & $20.8(4)$ \\
\hline C2A & $798.5(17)$ & $1771.6(18)$ & $2717.9(16)$ & $20.2(4)$ \\
\hline C3A & $-375.7(17)$ & $1772.3(18)$ & $2294.6(16)$ & $19.4(4)$ \\
\hline C4A & $-776.4(16)$ & $2857.6(17)$ & $1747.3(16)$ & $17.3(4)$ \\
\hline C5A & $-325.9(17)$ & $3177.6(17)$ & $902.5(15)$ & $17.5(4)$ \\
\hline C6A & $-763.0(18)$ & $4297.6(18)$ & $452.9(16)$ & $21.7(4)$ \\
\hline C7A & $-375.8(16)$ & $5237.9(18)$ & $1229.5(16)$ & $19.2(4)$ \\
\hline C8A & $807.1(16)$ & $5284.1(17)$ & $1671.4(15)$ & $16.8(4)$ \\
\hline C9A & $1288.6(16)$ & $4157.9(17)$ & $2094.6(15)$ & $16.4(4)$ \\
\hline C10A & $886.0(17)$ & $3166.9(17)$ & $1338.0(16)$ & $17.1(4)$ \\
\hline C11A & $2471.1(18)$ & $4244.6(19)$ & $2474(2)$ & $25.4(5)$ \\
\hline C12A & $2820.6(18)$ & $5103.6(19)$ & $3325.0(19)$ & $24.9(5)$ \\
\hline C13A & $2381.7(17)$ & $6264.2(17)$ & $3004.2(17)$ & $18.4(4)$ \\
\hline C14A & $1182.2(16)$ & $6214.7(16)$ & $2482.8(15)$ & $15.5(4)$ \\
\hline C15A & $786.3(16)$ & $6164.0(18)$ & $3396.5(15)$ & $17.9(4)$ \\
\hline C16A & $1459.1(18)$ & $7007.1(19)$ & $4155.7(16)$ & $22.3(4)$ \\
\hline C17A & $2543.7(17)$ & $6924.6(17)$ & $4037.2(16)$ & $20.3(4)$ \\
\hline C18A & $2921.2(19)$ & $6774(2)$ & $2304.4(18)$ & $25.9(5)$ \\
\hline C19A & $1373(2)$ & $3231(2)$ & $488.0(19)$ & $26.5(5)$ \\
\hline C20A & $3092.4(18)$ & $8013.0(18)$ & $4129.8(16)$ & $20.5(4)$ \\
\hline C21A & $4078.0(18)$ & $8104.5(19)$ & $4772.0(17)$ & $23.3(4)$ \\
\hline C22A & $2642.7(18)$ & $8967.3(19)$ & $3545.0(17)$ & $23.8(5)$ \\
\hline C23A & $3193(2)$ & $9908.3(19)$ & $3627.4(18)$ & $25.6(5)$ \\
\hline C24A & $4256.1(19)$ & $9972(2)$ & $4303.4(18)$ & $24.7(5)$ \\
\hline
\end{tabular}

Table S3. Anisotropic Displacement Parameters $\left(\AA 2 \times 10^{3}\right)$ for compound 2.

The Anisotropic displacement factor exponent takes the form: 
$-2 \pi^{2}\left[h^{2} a^{2} U_{11}+2 h k a * b * U_{12}+\ldots\right]$.

\begin{tabular}{|c|c|c|c|c|c|c|}
\hline Atom & $\mathbf{U}_{11}$ & $\mathbf{U}_{22}$ & $\mathbf{U}_{33}$ & $\mathbf{U}_{23}$ & $\mathbf{U}_{13}$ & $\mathbf{U}_{12}$ \\
\hline $\mathrm{O} 1 \mathrm{~W}$ & $44.8(11)$ & $25.4(8)$ & $53.7(11)$ & $15.1(8)$ & $36.0(9)$ & $15.1(8)$ \\
\hline O1 & $35.7(11)$ & $38.3(11)$ & $49.5(12)$ & $-11.5(9)$ & $14.4(9)$ & $-17.0(9)$ \\
\hline $\mathrm{O} 2$ & $34.6(10)$ & $30.8(9)$ & $47.8(10)$ & $-13.1(8)$ & $20.6(8)$ & $-8.2(8)$ \\
\hline $\mathrm{O} 3$ & $69.8(17)$ & $45.2(12)$ & $49.8(12)$ & $-15.5(10)$ & $41.3(12)$ & $-14.6(11)$ \\
\hline $\mathrm{O} 4$ & $50.1(12)$ & $42.5(11)$ & $36.2(9)$ & $0.7(8)$ & 24.2(9) & $-6.5(9)$ \\
\hline $\mathrm{O} 5$ & $13.8(8)$ & $35.0(9)$ & $22.0(7)$ & $-1.4(6)$ & $3.8(6)$ & $-4.9(6)$ \\
\hline O6 & $30.3(9)$ & $24.2(8)$ & $27.5(8)$ & $2.8(7)$ & $1.2(7)$ & $-1.6(7)$ \\
\hline O7 & $31.9(9)$ & $34.1(9)$ & $25.6(8)$ & $-2.5(7)$ & $9.8(7)$ & $-11.7(7)$ \\
\hline $\mathrm{C} 1$ & 29.1(13) & $38.7(14)$ & $26.0(11)$ & $-5.0(10)$ & $-0.6(9)$ & $-9.1(11)$ \\
\hline $\mathrm{C} 2$ & $17.3(11)$ & $38.9(14)$ & $49.8(14)$ & $-7.2(12)$ & $5.3(10)$ & $-5.5(10)$ \\
\hline $\mathrm{C} 3$ & $27.6(13)$ & $36.2(13)$ & $43.2(13)$ & $-7.7(11)$ & $17.7(11)$ & $-10.7(11)$ \\
\hline $\mathrm{C} 4$ & $26.5(12)$ & $28.9(12)$ & $33.0(12)$ & $-1.5(10)$ & $14.0(10)$ & $-7.9(10)$ \\
\hline C5 & $22.9(12)$ & $25.9(12)$ & $32.6(11)$ & $-6.3(9)$ & $12.7(9)$ & $-4.8(9)$ \\
\hline C6 & $20.7(12)$ & $27.8(12)$ & $44.6(14)$ & $0.4(10)$ & $6.0(10)$ & $-1.2(10)$ \\
\hline $\mathrm{C} 7$ & $21.2(11)$ & $31.9(13)$ & $31.7(12)$ & $1.4(10)$ & $0.3(9)$ & $-4.0(10)$ \\
\hline $\mathrm{C} 8$ & $16.7(10)$ & $25.2(11)$ & 21.2(9) & $-3.5(8)$ & $8.4(8)$ & $-3.2(8)$ \\
\hline C9 & $18.8(11)$ & $28.7(11)$ & 19.7(9) & $-3.7(9)$ & $7.2(8)$ & $-4.4(9)$ \\
\hline $\mathrm{C} 10$ & $24.7(11)$ & $28.0(11)$ & $22.3(10)$ & $-3.9(8)$ & $10.0(8)$ & $-6.8(9)$ \\
\hline $\mathrm{C} 11$ & $18.5(11)$ & $29.8(12)$ & $29.0(11)$ & $-1.4(9)$ & $1.0(9)$ & $-1.2(9)$ \\
\hline $\mathrm{C} 12$ & $18.2(11)$ & $30.6(12)$ & $38.0(12)$ & $-6.3(10)$ & $6.2(9)$ & $0.5(9)$ \\
\hline $\mathrm{C} 13$ & $18.3(11)$ & $28.1(11)$ & $26.6(10)$ & $-7.5(9)$ & $8.1(8)$ & $-3.2(9)$ \\
\hline $\mathrm{C} 14$ & $14.7(10)$ & $30.7(11)$ & $20.4(9)$ & $-5.2(8)$ & $8.2(8)$ & $-6.7(8)$ \\
\hline C15 & $31.3(13)$ & $46.1(15)$ & $23.9(11)$ & $-8.3(10)$ & $15.6(9)$ & $-16.9(11)$ \\
\hline $\mathrm{C} 16$ & $51.5(18)$ & $52.7(18)$ & $46.3(15)$ & $-27.2(14)$ & $37.3(14)$ & $-26.5(15)$ \\
\hline $\mathrm{C} 17$ & $23.7(12)$ & $34.4(13)$ & $42.8(13)$ & $-18.4(11)$ & $17.7(10)$ & $-8.6(10)$ \\
\hline C18 & $26.2(11)$ & 27.1(11) & $26.0(10)$ & $0.3(9)$ & $7.9(9)$ & $-4.7(9)$ \\
\hline C19 & $38.1(14)$ & $38.7(14)$ & $27.0(11)$ & $-6.2(10)$ & $16.8(10)$ & $-9.0(11)$ \\
\hline
\end{tabular}




\begin{tabular}{|c|c|c|c|c|c|c|}
\hline $\mathrm{C} 20$ & $21.7(11)$ & $27.5(11)$ & $27.0(10)$ & $-10.5(9)$ & $11.4(9)$ & $-3.0(9)$ \\
\hline $\mathrm{C} 21$ & $23.0(11)$ & $27.6(12)$ & $31.6(11)$ & $-4.8(9)$ & $0.7(9)$ & $2.4(9)$ \\
\hline $\mathrm{C} 22$ & $28.7(12)$ & $23.5(10)$ & 18.1(9) & $-2.1(8)$ & $8.6(8)$ & $-1.1(9)$ \\
\hline C23 & $22.0(10)$ & $22.6(10)$ & $16.9(9)$ & $-0.8(8)$ & $4.3(7)$ & $-0.4(8)$ \\
\hline C24 & $24.1(11)$ & $26.5(11)$ & 16.9(9) & $-2.8(8)$ & $7.3(8)$ & $-0.9(9)$ \\
\hline O6A & 20.8(8) & 22.7(8) & $32.4(8)$ & $-0.9(6)$ & $5.8(6)$ & $-3.8(6)$ \\
\hline O7A & $31.0(9)$ & 26.4(9) & 41.4(9) & $-4.1(7)$ & $14.2(7)$ & $-9.8(7)$ \\
\hline O5A & 24.4(8) & $15.5(7)$ & $18.3(6)$ & $3.5(5)$ & $10.3(6)$ & $4.8(6)$ \\
\hline $\mathrm{O} 4 \mathrm{~A}$ & $82.7(17)$ & $36.1(11)$ & $48.3(11)$ & $18.8(9)$ & $51.5(12)$ & $26.3(11)$ \\
\hline $\mathrm{O} 3 \mathrm{~A}$ & $35.0(10)$ & 32.1(9) & $37.0(9)$ & $1.7(7)$ & $24.2(8)$ & $5.8(8)$ \\
\hline O1A & $26.8(8)$ & 16.7(7) & 25.1(7) & $-1.5(6)$ & $7.5(6)$ & $-3.6(6)$ \\
\hline $\mathrm{O} 2 \mathrm{~A}$ & $34.6(9)$ & 17.6(7) & $15.8(7)$ & $-2.5(6)$ & $7.0(6)$ & $-1.8(6)$ \\
\hline $\mathrm{C} 1 \mathrm{~A}$ & $19.2(10)$ & $16.6(10)$ & $29.4(10)$ & $1.2(8)$ & $11.8(8)$ & $1.9(8)$ \\
\hline $\mathrm{C} 2 \mathrm{~A}$ & $19.8(10)$ & $18.6(9)$ & $22.0(9)$ & $5.0(8)$ & $6.6(8)$ & $0.0(8)$ \\
\hline C3A & $19.6(10)$ & $19.5(10)$ & $20.0(9)$ & $-0.5(8)$ & $7.7(8)$ & $-2.3(8)$ \\
\hline C4A & $18.0(9)$ & $18.0(10)$ & 17.3(9) & $-0.5(7)$ & $7.6(7)$ & $-1.2(7)$ \\
\hline C5A & $20.6(10)$ & 15.8(9) & $15.8(8)$ & $-1.4(7)$ & $5.6(7)$ & $1.4(8)$ \\
\hline C6A & $24.7(11)$ & $18.9(10)$ & $18.8(9)$ & $1.6(8)$ & $3.5(7)$ & $2.2(9)$ \\
\hline C7A & $18.8(10)$ & 16.8(9) & 20.9(9) & $0.9(7)$ & $5.2(8)$ & $3.3(8)$ \\
\hline C8A & $19.9(10)$ & 15.3(9) & $17.4(8)$ & $1.7(7)$ & $9.2(7)$ & $2.0(8)$ \\
\hline C9A & $15.9(9)$ & 13.2(9) & 21.2(9) & $2.0(7)$ & $7.5(7)$ & $1.1(7)$ \\
\hline C10A & $20.2(10)$ & 14.1(9) & 20.9(9) & $0.5(7)$ & $12.2(8)$ & $1.3(7)$ \\
\hline C11A & $16.9(10)$ & $18.2(10)$ & $41.3(12)$ & $-3.5(9)$ & $9.8(9)$ & $0.8(8)$ \\
\hline C12A & $17.0(10)$ & $18.4(10)$ & $35.3(11)$ & $0.2(9)$ & $3.4(8)$ & $0.7(8)$ \\
\hline C13A & $16.5(10)$ & 15.6(9) & $25.5(10)$ & $0.1(7)$ & $10.4(8)$ & $-0.5(8)$ \\
\hline C14A & $17.5(9)$ & $13.6(9)$ & 18.3(9) & $1.7(7)$ & $9.9(7)$ & $-0.7(7)$ \\
\hline C15A & $19.3(10)$ & 19.5(9) & $17.6(9)$ & $2.1(7)$ & $9.7(7)$ & $0.5(7)$ \\
\hline C16A & $26.6(11)$ & $22.9(10)$ & $19.4(9)$ & $-1.5(8)$ & $10.1(8)$ & $-3.4(8)$ \\
\hline C17A & $21.3(10)$ & $17.8(10)$ & $20.5(9)$ & $3.0(7)$ & $5.5(8)$ & $-1.8(8)$ \\
\hline
\end{tabular}




\begin{tabular}{|l|l|l|l|l|l|l|}
\hline C18A & $25.5(11)$ & $24.8(11)$ & $34.1(11)$ & $-4.4(9)$ & $18.8(9)$ & $-6.1(9)$ \\
\hline C19A & $34.5(13)$ & $23.0(11)$ & $31.4(11)$ & $-2.4(9)$ & $23.4(10)$ & $-1.6(9)$ \\
\hline C20A & $21.6(11)$ & $17.7(10)$ & $22.1(9)$ & $0.7(8)$ & $7.2(8)$ & $-1.7(8)$ \\
\hline C21A & $21.8(11)$ & $19.8(10)$ & $27.1(10)$ & $1.6(8)$ & $6.5(8)$ & $-0.3(8)$ \\
\hline C22A & $21.7(11)$ & $20.3(10)$ & $27.1(10)$ & $2.6(8)$ & $5.1(8)$ & $0.6(8)$ \\
\hline C23A & $26.7(12)$ & $17.4(10)$ & $31.3(11)$ & $2.8(8)$ & $7.6(9)$ & $0.2(9)$ \\
\hline C24A & $26.1(12)$ & $21.9(11)$ & $28.2(10)$ & $-4.1(9)$ & $12.1(9)$ & $-2.7(9)$ \\
\hline
\end{tabular}

Table S4. Bond Lengths for compound 2.

\begin{tabular}{|l|l|l|l|l|l|l|}
\hline \multicolumn{1}{|c|}{ Atom } & \multicolumn{1}{|c|}{ Atom } & \multicolumn{1}{|c|}{ Length/A } & & \multicolumn{1}{|c|}{ Atom } & \multicolumn{1}{|c|}{ Atom } & \multicolumn{1}{|c|}{ Length/A } \\
\hline O1 & C3 & $1.441(3)$ & & O6A & C21A & $1.367(3)$ \\
\hline O2 & C5 & $1.461(3)$ & & O6A & C24A & $1.372(3)$ \\
\hline O3 & C19 & $1.332(4)$ & & O7A & C24A & $1.211(3)$ \\
\hline O4 & C19 & $1.202(4)$ & & O5A & C14A & $1.446(2)$ \\
\hline O5 & C14 & $1.449(3)$ & & O4A & C19A & $1.311(3)$ \\
\hline O6 & C21 & $1.367(3)$ & & O3A & C19A & $1.214(3)$ \\
\hline O6 & C24 & $1.380(3)$ & & O1A & C3A & $1.434(2)$ \\
\hline O7 & C24 & $1.217(3)$ & & O2A & C5A & $1.444(2)$ \\
\hline C1 & C2 & $1.528(4)$ & & C1A & C2A & $1.525(3)$ \\
\hline C1 & C10 & $1.547(3)$ & & C1A & C10A & $1.550(3)$ \\
\hline C2 & C3 & $1.525(4)$ & & C2A & C3A & $1.519(3)$ \\
\hline C3 & C4 & $1.520(4)$ & & C3A & C4A & $1.527(3)$ \\
\hline C4 & C5 & $1.520(3)$ & & C4A & C5A & $1.539(3)$ \\
\hline C5 & C6 & $1.529(3)$ & & C5A & C6A & $1.532(3)$ \\
\hline C5 & C10 & $1.564(3)$ & & C5A & C10A & $1.568(3)$ \\
\hline C6 & C7 & $1.529(4)$ & & C6A & C7A & $1.534(3)$ \\
\hline C7 & C8 & $1.533(3)$ & & C7A & C8A & $1.532(3)$ \\
\hline C8 & C9 & $1.542(3)$ & & C8A & C9A & $1.546(3)$ \\
\hline C8 & C14 & $1.546(3)$ & & C8A & C14A & $1.552(3)$ \\
\hline
\end{tabular}




\begin{tabular}{|l|l|l|l|l|l|l|}
\hline $\mathrm{C} 9$ & $\mathrm{C} 10$ & $1.566(3)$ & & $\mathrm{C} 9 \mathrm{~A}$ & $\mathrm{C} 10 \mathrm{~A}$ & $1.567(3)$ \\
\hline $\mathrm{C} 9$ & $\mathrm{C} 11$ & $1.531(3)$ & & $\mathrm{C} 9 \mathrm{~A}$ & $\mathrm{C} 11 \mathrm{~A}$ & $1.533(3)$ \\
\hline $\mathrm{C} 10$ & $\mathrm{C} 19$ & $1.557(3)$ & & $\mathrm{C} 10 \mathrm{~A}$ & $\mathrm{C} 19 \mathrm{~A}$ & $1.532(3)$ \\
\hline $\mathrm{C} 11$ & $\mathrm{C} 12$ & $1.536(3)$ & & $\mathrm{C} 11 \mathrm{~A}$ & $\mathrm{C} 12 \mathrm{~A}$ & $1.523(3)$ \\
\hline $\mathrm{C} 12$ & $\mathrm{C} 13$ & $1.541(3)$ & & $\mathrm{C} 12 \mathrm{~A}$ & $\mathrm{C} 13 \mathrm{~A}$ & $1.538(3)$ \\
\hline $\mathrm{C} 13$ & $\mathrm{C} 14$ & $1.538(3)$ & & $\mathrm{C} 13 \mathrm{~A}$ & $\mathrm{C} 14 \mathrm{~A}$ & $1.561(3)$ \\
\hline $\mathrm{C} 13$ & $\mathrm{C} 17$ & $1.576(3)$ & & $\mathrm{C} 13 \mathrm{~A}$ & $\mathrm{C} 17 \mathrm{~A}$ & $1.584(3)$ \\
\hline $\mathrm{C} 13$ & $\mathrm{C} 18$ & $1.529(3)$ & & $\mathrm{C} 13 \mathrm{~A}$ & $\mathrm{C} 18 \mathrm{~A}$ & $1.528(3)$ \\
\hline $\mathrm{C} 14$ & $\mathrm{C} 15$ & $1.535(3)$ & & $\mathrm{C} 14 \mathrm{~A}$ & $\mathrm{C} 15 \mathrm{~A}$ & $1.530(3)$ \\
\hline $\mathrm{C} 15$ & $\mathrm{C} 16$ & $1.552(4)$ & & $\mathrm{C} 15 \mathrm{~A}$ & $\mathrm{C} 16 \mathrm{~A}$ & $1.533(3)$ \\
\hline $\mathrm{C} 16$ & $\mathrm{C} 17$ & $1.540(4)$ & & $\mathrm{C} 16 \mathrm{~A}$ & $\mathrm{C} 17 \mathrm{~A}$ & $1.553(3)$ \\
\hline $\mathrm{C} 17$ & $\mathrm{C} 20$ & $1.513(3)$ & & $\mathrm{C} 17 \mathrm{~A}$ & $\mathrm{C} 20 \mathrm{~A}$ & $1.505(3)$ \\
\hline $\mathrm{C} 20$ & $\mathrm{C} 21$ & $1.348(4)$ & & $\mathrm{C} 20 \mathrm{~A}$ & $\mathrm{C} 21 \mathrm{~A}$ & $1.351(3)$ \\
\hline $\mathrm{C} 20$ & $\mathrm{C} 22$ & $1.430(3)$ & & $\mathrm{C} 20 \mathrm{~A}$ & $\mathrm{C} 22 \mathrm{~A}$ & $1.429(3)$ \\
\hline $\mathrm{C} 22$ & $\mathrm{C} 23$ & $1.357(3)$ & & $\mathrm{C} 22 \mathrm{~A}$ & $\mathrm{C} 23 \mathrm{~A}$ & $1.353(3)$ \\
\hline $\mathrm{C} 23$ & $\mathrm{C} 24$ & $1.420(3)$ & & $\mathrm{C} 23 \mathrm{~A}$ & $\mathrm{C} 24 \mathrm{~A}$ & $1.447(3)$ \\
\hline
\end{tabular}

Table S5. Bond Angles for compound 2.

\begin{tabular}{|l|l|l|l|l|l|l|l|l|}
\hline Atom & Atom & Atom & \multicolumn{1}{|c|}{ Angle/ $^{\circ}$} & & Atom & Atom & Atom & \multicolumn{1}{|c|}{ Angle/ $^{\circ}$} \\
\hline C21 & O6 & C24 & $121.32(19)$ & C21A & O6A & C24A & $121.80(18)$ \\
\hline C2 & $\mathrm{C} 1$ & $\mathrm{C} 10$ & $112.0(2)$ & $\mathrm{C} 2 \mathrm{~A}$ & $\mathrm{C} 1 \mathrm{~A}$ & $\mathrm{C} 10 \mathrm{~A}$ & $114.02(18)$ \\
\hline $\mathrm{C} 3$ & $\mathrm{C} 2$ & $\mathrm{C} 1$ & $111.4(2)$ & $\mathrm{C} 3 \mathrm{~A}$ & $\mathrm{C} 2 \mathrm{~A}$ & $\mathrm{C} 1 \mathrm{~A}$ & $111.06(17)$ \\
\hline O1 & $\mathrm{C} 3$ & $\mathrm{C} 2$ & $110.5(2)$ & $\mathrm{O} 1 \mathrm{~A}$ & $\mathrm{C} 3 \mathrm{~A}$ & $\mathrm{C} 2 \mathrm{~A}$ & $111.47(18)$ \\
\hline O1 & $\mathrm{C} 3$ & $\mathrm{C} 4$ & $106.3(2)$ & $\mathrm{O} 1 \mathrm{~A}$ & $\mathrm{C} 3 \mathrm{~A}$ & $\mathrm{C} 4 \mathrm{~A}$ & $106.73(16)$ \\
\hline $\mathrm{C} 4$ & $\mathrm{C} 3$ & $\mathrm{C} 2$ & $111.8(2)$ & $\mathrm{C} 2 \mathrm{~A}$ & $\mathrm{C} 3 \mathrm{~A}$ & $\mathrm{C} 4 \mathrm{~A}$ & $110.56(17)$ \\
\hline $\mathrm{C} 3$ & $\mathrm{C} 4$ & $\mathrm{C} 5$ & $113.3(2)$ & $\mathrm{C} 3 \mathrm{~A}$ & $\mathrm{C} 4 \mathrm{~A}$ & $\mathrm{C} 5 \mathrm{~A}$ & $115.01(18)$ \\
\hline O2 & $\mathrm{C} 5$ & $\mathrm{C} 4$ & $107.0(2)$ & $\mathrm{O} 2 \mathrm{~A}$ & $\mathrm{C} 5 \mathrm{~A}$ & $\mathrm{C} 4 \mathrm{~A}$ & $108.65(17)$ \\
\hline O2 & $\mathrm{C} 5$ & $\mathrm{C} 6$ & $105.4(2)$ & $\mathrm{O} 2 \mathrm{~A}$ & $\mathrm{C} 5 \mathrm{~A}$ & $\mathrm{C} 6 \mathrm{~A}$ & $107.10(16)$ \\
\hline O2 & $\mathrm{C} 5$ & $\mathrm{C} 10$ & $110.81(19)$ & $\mathrm{O} 2 \mathrm{~A}$ & $\mathrm{C} 5 \mathrm{~A}$ & $\mathrm{C} 10 \mathrm{~A}$ & $108.69(16)$ \\
\hline
\end{tabular}




\begin{tabular}{|c|c|c|c|c|c|c|c|}
\hline $\mathrm{C} 4$ & $\mathrm{C} 5$ & C6 & $112.6(2)$ & $\mathrm{C} 4 \mathrm{~A}$ & C5A & C10A & $110.48(16)$ \\
\hline $\mathrm{C} 4$ & C5 & $\mathrm{C} 10$ & $111.5(2)$ & C6A & $\mathrm{C} 5 \mathrm{~A}$ & $\mathrm{C} 4 \mathrm{~A}$ & $109.20(17)$ \\
\hline C6 & $\mathrm{C} 5$ & C10 & 109.32(19) & C6A & $\mathrm{C} 5 \mathrm{~A}$ & C10A & $112.60(17)$ \\
\hline C5 & C6 & $\mathrm{C} 7$ & $113.1(2)$ & $\mathrm{C} 5 \mathrm{~A}$ & C6A & C7A & $112.40(16)$ \\
\hline C6 & $\mathrm{C} 7$ & $\mathrm{C} 8$ & $112.2(2)$ & C8A & C7A & C6A & $112.28(18)$ \\
\hline $\mathrm{C} 7$ & $\mathrm{C} 8$ & C9 & $109.28(19)$ & C7A & C8A & C9A & $112.34(17)$ \\
\hline $\mathrm{C} 7$ & $\mathrm{C} 8$ & C14 & $111.23(18)$ & C7A & C8A & C14A & $111.46(17)$ \\
\hline C9 & $\mathrm{C} 8$ & C14 & $114.17(19)$ & C9A & C8A & C14A & $112.03(16)$ \\
\hline $\mathrm{C} 8$ & C9 & $\mathrm{C} 10$ & $110.08(19)$ & C8A & C9A & C10A & $114.64(16)$ \\
\hline C11 & C9 & $\mathrm{C} 8$ & $112.82(19)$ & C11A & C9A & C8A & $109.77(17)$ \\
\hline C11 & C9 & C10 & $114.24(18)$ & C11A & C9A & C10A & $112.19(17)$ \\
\hline $\mathrm{C} 1$ & $\mathrm{C} 10$ & C5 & $108.9(2)$ & $\mathrm{C} 1 \mathrm{~A}$ & C10A & $\mathrm{C} 5 \mathrm{~A}$ & $109.04(17)$ \\
\hline $\mathrm{C} 1$ & $\mathrm{C} 10$ & C9 & $111.7(2)$ & C1A & C10A & C9A & $111.49(16)$ \\
\hline $\mathrm{C} 1$ & $\mathrm{C} 10$ & C19 & 107.02(19) & C9A & C10A & $\mathrm{C} 5 \mathrm{~A}$ & $109.98(16)$ \\
\hline C5 & $\mathrm{C} 10$ & C9 & $108.88(17)$ & C19A & C10A & $\mathrm{C} 1 \mathrm{~A}$ & $104.86(17)$ \\
\hline C19 & $\mathrm{C} 10$ & C5 & $109.6(2)$ & C19A & C10A & C5A & $112.56(18)$ \\
\hline C19 & $\mathrm{C} 10$ & C9 & $110.7(2)$ & C19A & C10A & C9A & $108.84(17)$ \\
\hline C9 & C11 & $\mathrm{C} 12$ & $110.22(19)$ & $\mathrm{C} 12 \mathrm{~A}$ & C11A & C9A & $109.60(19)$ \\
\hline C11 & $\mathrm{C} 12$ & C13 & $113.2(2)$ & C11A & C12A & C13A & $114.44(18)$ \\
\hline $\mathrm{C} 12$ & C13 & C17 & $105.60(19)$ & $\mathrm{C} 12 \mathrm{~A}$ & C13A & C14A & $110.38(17)$ \\
\hline C14 & C13 & $\mathrm{C} 12$ & $109.0(2)$ & $\mathrm{C} 12 \mathrm{~A}$ & C13A & C17A & $106.34(17)$ \\
\hline C14 & $\mathrm{C} 13$ & $\mathrm{C} 17$ & $104.58(19)$ & C14A & C13A & C17A & $104.11(16)$ \\
\hline C18 & C13 & $\mathrm{C} 12$ & $110.1(2)$ & C18A & C13A & C12A & $109.09(19)$ \\
\hline $\mathrm{C} 18$ & $\mathrm{C} 13$ & C14 & $113.5(2)$ & C18A & C13A & C14A & $112.49(17)$ \\
\hline $\mathrm{C} 18$ & $\mathrm{C} 13$ & C17 & $113.6(2)$ & C18A & C13A & C17A & $114.19(18)$ \\
\hline O5 & C14 & $\mathrm{C} 8$ & $106.83(18)$ & O5A & C14A & C8A & $109.05(15)$ \\
\hline O5 & C14 & C13 & $106.30(18)$ & O5A & C14A & C13A & $109.33(16)$ \\
\hline O5 & C14 & C15 & 109.74(17) & O5A & C14A & C15A & $104.49(16)$ \\
\hline C13 & C14 & $\mathrm{C} 8$ & $114.47(17)$ & C8A & C14A & C13A & $113.89(17)$ \\
\hline
\end{tabular}




\begin{tabular}{|l|l|l|l|l|l|l|l|}
\hline $\mathrm{C} 15$ & $\mathrm{C} 14$ & $\mathrm{C} 8$ & $115.04(19)$ & $\mathrm{C} 15 \mathrm{~A}$ & $\mathrm{C} 14 \mathrm{~A}$ & $\mathrm{C} 8 \mathrm{~A}$ & $116.13(17)$ \\
\hline $\mathrm{C} 15$ & $\mathrm{C} 14$ & $\mathrm{C} 13$ & $104.1(2)$ & $\mathrm{C} 15 \mathrm{~A}$ & $\mathrm{C} 14 \mathrm{~A}$ & $\mathrm{C} 13 \mathrm{~A}$ & $103.37(15)$ \\
\hline $\mathrm{C} 14$ & $\mathrm{C} 15$ & $\mathrm{C} 16$ & $106.9(2)$ & $\mathrm{C} 14 \mathrm{~A}$ & $\mathrm{C} 15 \mathrm{~A}$ & $\mathrm{C} 16 \mathrm{~A}$ & $103.70(17)$ \\
\hline $\mathrm{C} 17$ & $\mathrm{C} 16$ & $\mathrm{C} 15$ & $107.4(2)$ & $\mathrm{C} 15 \mathrm{~A}$ & $\mathrm{C} 16 \mathrm{~A}$ & $\mathrm{C} 17 \mathrm{~A}$ & $105.36(17)$ \\
\hline $\mathrm{C} 16$ & $\mathrm{C} 17$ & $\mathrm{C} 13$ & $104.1(2)$ & $\mathrm{C} 16 \mathrm{~A}$ & $\mathrm{C} 17 \mathrm{~A}$ & $\mathrm{C} 13 \mathrm{~A}$ & $105.96(17)$ \\
\hline $\mathrm{C} 20$ & $\mathrm{C} 17$ & $\mathrm{C} 13$ & $115.6(2)$ & $\mathrm{C} 20 \mathrm{~A}$ & $\mathrm{C} 17 \mathrm{~A}$ & $\mathrm{C} 13 \mathrm{~A}$ & $115.93(18)$ \\
\hline $\mathrm{C} 20$ & $\mathrm{C} 17$ & $\mathrm{C} 16$ & $113.9(2)$ & $\mathrm{C} 20 \mathrm{~A}$ & $\mathrm{C} 17 \mathrm{~A}$ & $\mathrm{C} 16 \mathrm{~A}$ & $113.81(18)$ \\
\hline O3 & $\mathrm{C} 19$ & $\mathrm{C} 10$ & $116.7(2)$ & $\mathrm{O} 4 \mathrm{~A}$ & $\mathrm{C} 19 \mathrm{~A}$ & $\mathrm{C} 10 \mathrm{~A}$ & $114.9(2)$ \\
\hline $\mathrm{O} 4$ & $\mathrm{C} 19$ & $\mathrm{O} 3$ & $118.8(2)$ & $\mathrm{O} 3 \mathrm{~A}$ & $\mathrm{C} 19 \mathrm{~A}$ & $\mathrm{O} 4 \mathrm{~A}$ & $121.4(2)$ \\
\hline O4 & $\mathrm{C} 19$ & $\mathrm{C} 10$ & $124.4(2)$ & $\mathrm{O} 3 \mathrm{~A}$ & $\mathrm{C} 19 \mathrm{~A}$ & $\mathrm{C} 10 \mathrm{~A}$ & $123.4(2)$ \\
\hline $\mathrm{C} 21$ & $\mathrm{C} 20$ & $\mathrm{C} 17$ & $119.8(2)$ & $\mathrm{C} 21 \mathrm{~A}$ & $\mathrm{C} 20 \mathrm{~A}$ & $\mathrm{C} 17 \mathrm{~A}$ & $119.4(2)$ \\
\hline $\mathrm{C} 21$ & $\mathrm{C} 20$ & $\mathrm{C} 22$ & $116.3(2)$ & $\mathrm{C} 21 \mathrm{~A}$ & $\mathrm{C} 20 \mathrm{~A}$ & $\mathrm{C} 22 \mathrm{~A}$ & $116.9(2)$ \\
\hline $\mathrm{C} 22$ & $\mathrm{C} 20$ & $\mathrm{C} 17$ & $123.9(2)$ & $\mathrm{C} 22 \mathrm{~A}$ & $\mathrm{C} 20 \mathrm{~A}$ & $\mathrm{C} 17 \mathrm{~A}$ & $123.7(2)$ \\
\hline $\mathrm{C} 20$ & $\mathrm{C} 21$ & $\mathrm{O} 6$ & $123.5(2)$ & $\mathrm{C} 20 \mathrm{~A}$ & $\mathrm{C} 21 \mathrm{~A}$ & $\mathrm{O} 6 \mathrm{~A}$ & $123.2(2)$ \\
\hline $\mathrm{C} 23$ & $\mathrm{C} 22$ & $\mathrm{C} 20$ & $121.1(2)$ & $\mathrm{C} 23 \mathrm{~A}$ & $\mathrm{C} 22 \mathrm{~A}$ & $\mathrm{C} 20 \mathrm{~A}$ & $120.9(2)$ \\
\hline $\mathrm{C} 22$ & $\mathrm{C} 23$ & $\mathrm{C} 24$ & $121.1(2)$ & $\mathrm{C} 22 \mathrm{~A}$ & $\mathrm{C} 23 \mathrm{~A}$ & $\mathrm{C} 24 \mathrm{~A}$ & $120.9(2)$ \\
\hline O6 & $\mathrm{C} 24$ & $\mathrm{C} 23$ & $116.5(2)$ & $\mathrm{O} 6 \mathrm{~A}$ & $\mathrm{C} 24 \mathrm{~A}$ & $\mathrm{C} 23 \mathrm{~A}$ & $116.2(2)$ \\
\hline O7 & $\mathrm{C} 24$ & $\mathrm{O} 6$ & $117.4(2)$ & $\mathrm{O} 7 \mathrm{~A}$ & $\mathrm{C} 24 \mathrm{~A}$ & $\mathrm{O} 6 \mathrm{~A}$ & $117.1(2)$ \\
\hline O7 & $\mathrm{C} 24$ & $\mathrm{C} 23$ & $126.0(2)$ & $\mathrm{O} 7 \mathrm{~A}$ & $\mathrm{C} 24 \mathrm{~A}$ & $\mathrm{C} 23 \mathrm{~A}$ & $126.6(2)$ \\
\hline
\end{tabular}

Table S6. Hydrogen Atom Coordinates $\left(\AA \times 10^{4}\right)$ and Isotropic Displacement Parameters $\left(\AA 2 \times 10^{3}\right)$ for compound 2.

\begin{tabular}{|l|l|l|l|l|}
\hline \multicolumn{1}{|c|}{ Atom } & \multicolumn{1}{c|}{$\boldsymbol{x}$} & \multicolumn{1}{c|}{$\boldsymbol{y}$} & \multicolumn{1}{c|}{$\boldsymbol{~}$} & \multicolumn{1}{c|}{ U(eq) } \\
\hline H1WA & -60.87 & 8371.86 & 2252.2 & 55 \\
\hline H1WB & -833.51 & 8552.57 & 2711.45 & 55 \\
\hline H1 & 6456.58 & 7911.37 & 9092.98 & 62 \\
\hline H2 & 4489.23 & 7690.42 & 8952.58 & 54 \\
\hline H3 & 3775.53 & 6686.28 & 9867.49 & 74 \\
\hline H5 & 1316.61 & 3361.68 & 5867.55 & 36 \\
\hline
\end{tabular}




\begin{tabular}{|c|c|c|c|c|}
\hline $\mathrm{H} 1 \mathrm{~A}$ & 5425.62 & 6143.36 & 9977.68 & 40 \\
\hline H1B & 5611.32 & 4849.36 & 9902.29 & 40 \\
\hline $\mathrm{H} 2 \mathrm{~A}$ & 6805.98 & 5876.38 & 9345.66 & 44 \\
\hline $\mathrm{H} 2 \mathrm{~B}$ & 6097.11 & 5116.43 & 8432.46 & 44 \\
\hline $\mathrm{H} 3 \mathrm{~A}$ & 6182.37 & 6865.03 & 7746.21 & 41 \\
\hline $\mathrm{H} 4 \mathrm{~A}$ & 4540.91 & 6116.39 & 6996.35 & 34 \\
\hline $\mathrm{H} 4 \mathrm{~B}$ & 4402.03 & 7405.14 & 7149.39 & 34 \\
\hline H6A & 2563.43 & 6961.17 & 6968.35 & 39 \\
\hline H6B & 2317.72 & 6288.49 & 7857.6 & 39 \\
\hline H7A & 2954.03 & 5337.38 & 6260.96 & 36 \\
\hline H7B & 1834.91 & 5202.82 & 6372.14 & 36 \\
\hline H8 & 2547.6 & 4173.68 & 7894.8 & 25 \\
\hline H9 & 4476.86 & 4535.96 & 7581.12 & 27 \\
\hline H11A & 4269.52 & 3126.46 & 9162.42 & 33 \\
\hline H11B & 5329.72 & 3400.62 & 8961.17 & 33 \\
\hline $\mathrm{H} 12 \mathrm{~A}$ & 4697 & 1666.71 & 8224.51 & 36 \\
\hline H12B & 4715.81 & 2508.36 & 7338.42 & 36 \\
\hline $\mathrm{H} 15 \mathrm{~A}$ & 3694.5 & 3683.43 & 5928.28 & 39 \\
\hline H15B & 2510.77 & 3567.31 & 5193.77 & 39 \\
\hline H16A & 2762.45 & 1805 & 4858.3 & 54 \\
\hline H16B & 3973.38 & 1969.25 & 5479.42 & 54 \\
\hline H17 & 3805.08 & 822.34 & 6640.51 & 38 \\
\hline $\mathrm{H} 18 \mathrm{~A}$ & 2798.96 & 2229.14 & 8599.53 & 40 \\
\hline H18B & 3031.84 & 993.23 & 8338.38 & 40 \\
\hline $\mathrm{H} 18 \mathrm{C}$ & 1969.16 & 1575.33 & 7683.79 & 40 \\
\hline $\mathrm{H} 21$ & 2948.62 & -694.49 & 6978.29 & 35 \\
\hline $\mathrm{H} 22$ & 1199.05 & 1508.72 & 4974.1 & 28 \\
\hline $\mathrm{H} 23$ & -168.07 & 307.76 & 4551.07 & 25 \\
\hline H5A & 752.8 & 7228.41 & 1372.17 & 28 \\
\hline
\end{tabular}




\begin{tabular}{|c|c|c|c|c|}
\hline H4AA & 1471.61 & 4118.66 & -500.76 & 72 \\
\hline H1AA & -611.09 & 298.54 & 1840.38 & 35 \\
\hline $\mathrm{H} 2 \mathrm{AA}$ & -735.79 & 1765.02 & 321.03 & 35 \\
\hline $\mathrm{H} 1 \mathrm{AB}$ & 1069.33 & 1457.48 & 1354.84 & 25 \\
\hline $\mathrm{H} 1 \mathrm{AC}$ & 2018.81 & 2062.34 & 2186.68 & 25 \\
\hline $\mathrm{H} 2 \mathrm{AB}$ & 1046.01 & 1036.96 & 3007.48 & 24 \\
\hline $\mathrm{H} 2 \mathrm{AC}$ & 1037.72 & 2318.55 & 3280.23 & 24 \\
\hline H3AA & -654.06 & 1669.89 & 2873.2 & 23 \\
\hline $\mathrm{H} 4 \mathrm{AB}$ & -619.42 & 3453.3 & 2267 & 21 \\
\hline $\mathrm{H} 4 \mathrm{AC}$ & -1538.11 & 2809.49 & 1435.12 & 21 \\
\hline H6AA & -1527.65 & 4270.11 & 228.18 & 26 \\
\hline H6AB & -561.88 & 4448.89 & -160.57 & 26 \\
\hline H7AA & -639.65 & 5946.41 & 887.29 & 23 \\
\hline $\mathrm{H} 7 \mathrm{AB}$ & -655.59 & 5139.02 & 1799.69 & 23 \\
\hline H8A & 1057.12 & 5474.74 & 1088.61 & 20 \\
\hline H9A & 1086 & 4001.17 & 2713.87 & 20 \\
\hline $\mathrm{H} 11 \mathrm{C}$ & 2779.19 & 3521.42 & 2738.42 & 31 \\
\hline H11D & 2708.66 & 4456.73 & 1895.94 & 31 \\
\hline $\mathrm{H} 12 \mathrm{C}$ & 3585.2 & 5144.5 & 3566.78 & 30 \\
\hline H12D & 2611.87 & 4857.24 & 3911.88 & 30 \\
\hline $\mathrm{H} 15 \mathrm{C}$ & 877.51 & 5417.65 & 3702.67 & 21 \\
\hline H15D & 45.78 & 6367.73 & 3185.34 & 21 \\
\hline H16C & 1498.57 & 6829.15 & 4868.75 & 27 \\
\hline H16D & 1172.77 & 7758.34 & 3985.88 & 27 \\
\hline H17A & 2976.93 & 6450.65 & 4613.07 & 24 \\
\hline H18D & 2779.14 & 6327.02 & 1680.32 & 39 \\
\hline $\mathrm{H} 18 \mathrm{E}$ & 3668.72 & 6797.79 & 2667.92 & 39 \\
\hline $\mathrm{H} 18 \mathrm{~F}$ & 2661.63 & 7522.94 & 2116.3 & 39 \\
\hline $\mathrm{H} 21 \mathrm{~A}$ & 4390.09 & 7483.65 & 5175.62 & 28 \\
\hline
\end{tabular}




\begin{tabular}{|l|l|l|l|l|}
\hline H22A & 1947.65 & 8940.82 & 3092.15 & 29 \\
\hline H23A & 2877.3 & 10535.29 & 3236.08 & 31 \\
\hline
\end{tabular}


2. Spectra of Compounds 1-3

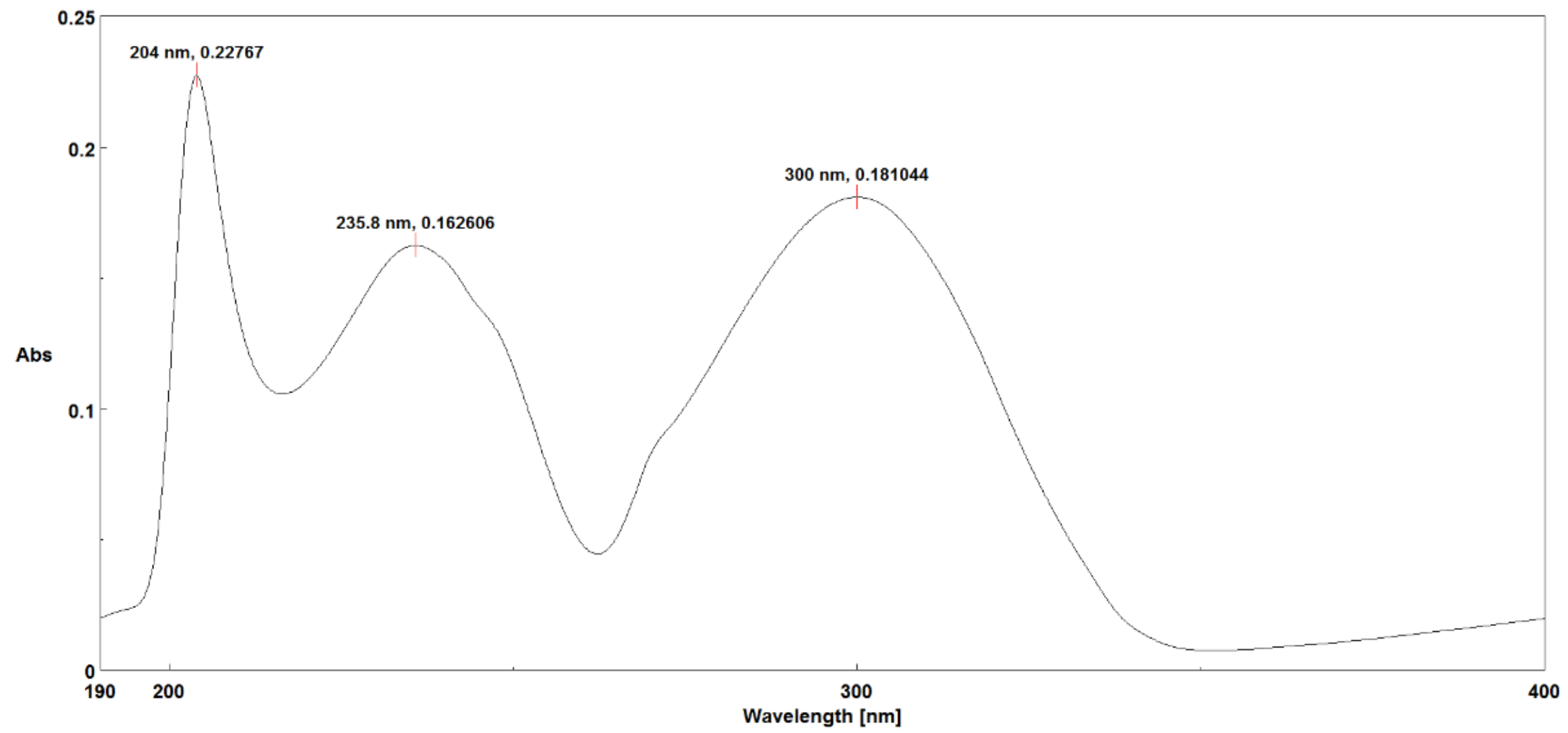

Figure S2. The UV spectrum of compound $\mathbf{1}$ in $\mathrm{MeOH}$. 


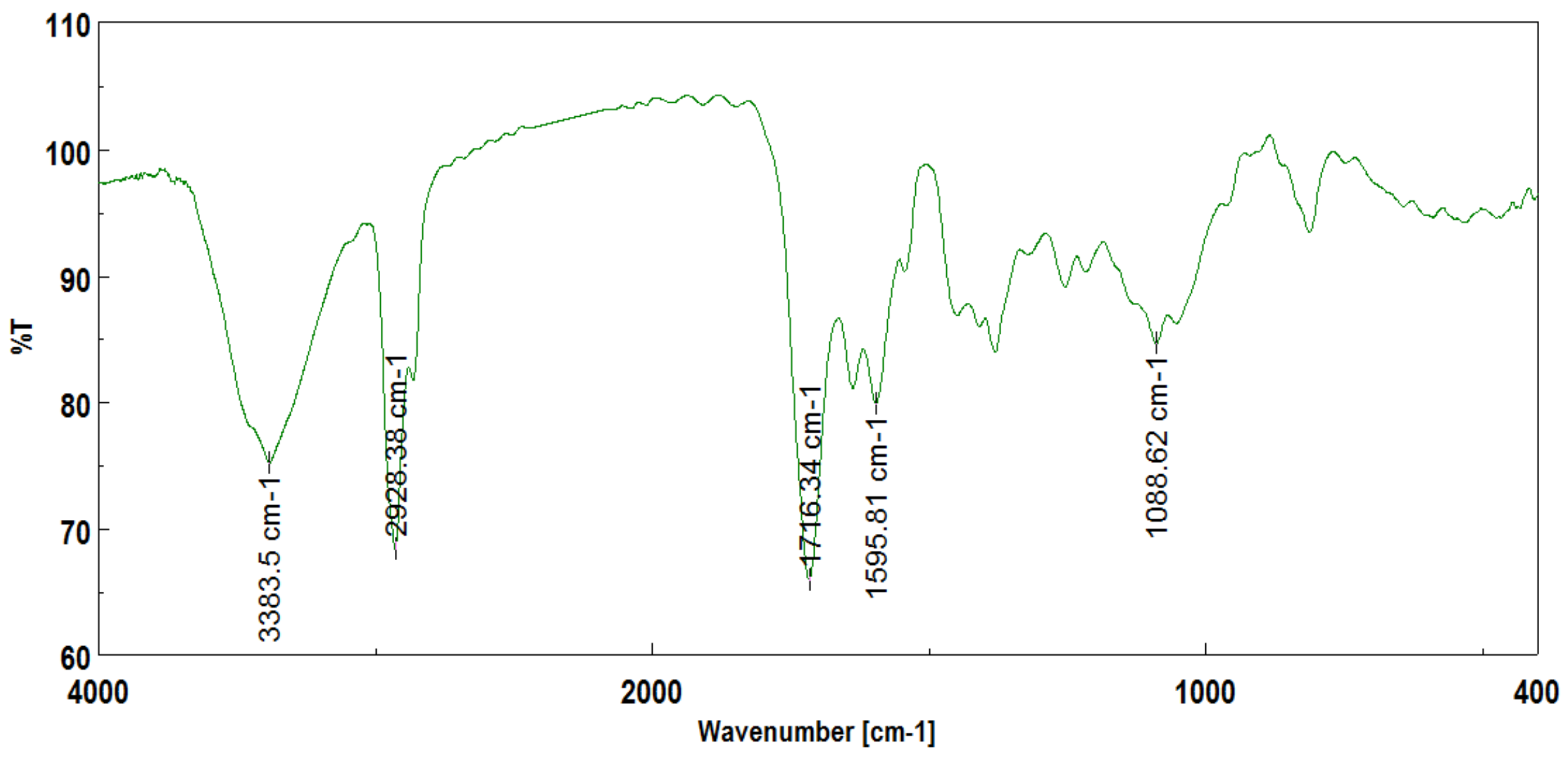

Figure S3. The IR spectrum of compound 1. 


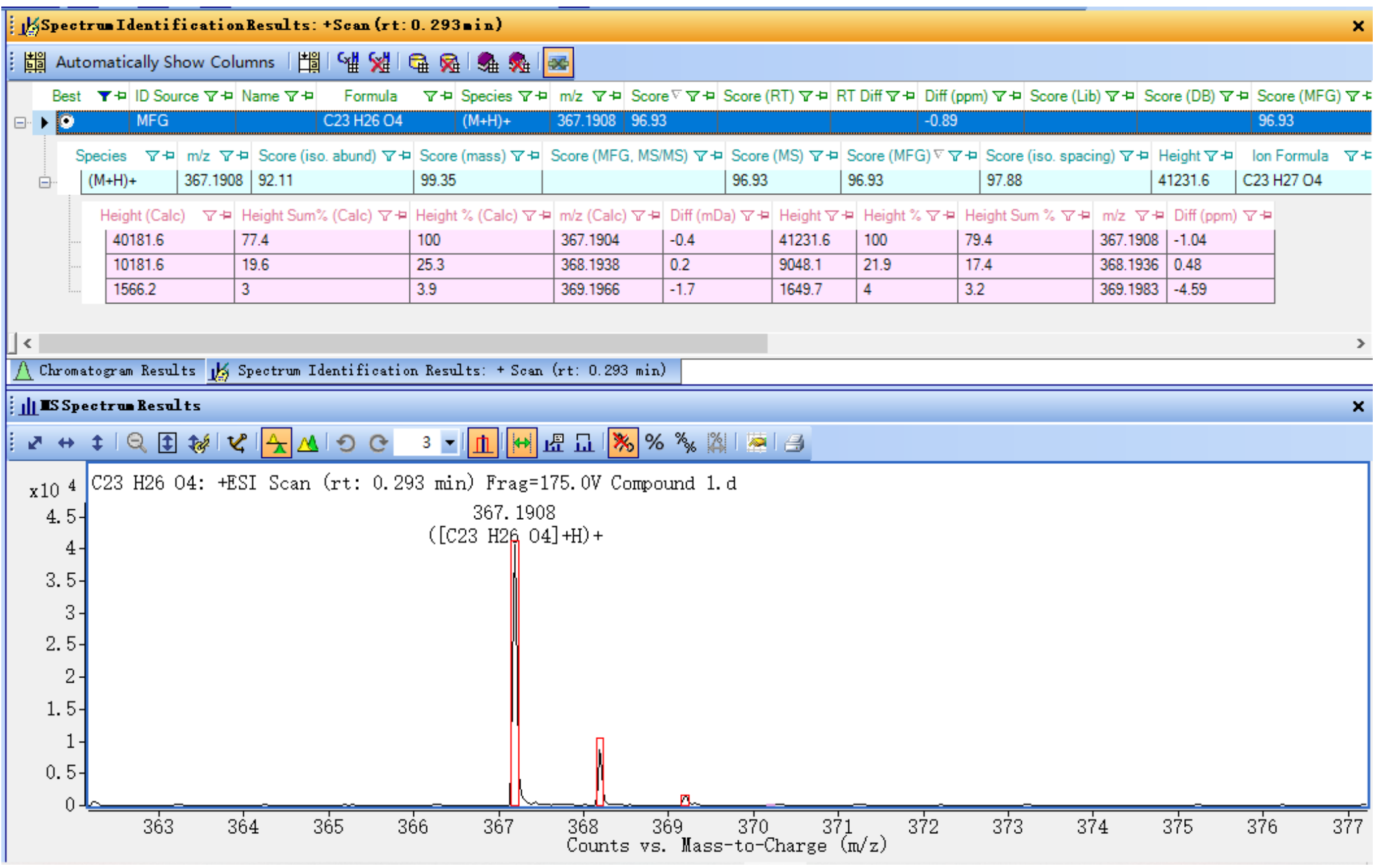

Figure S4. The HRESIMS spectrum of compound 1. 


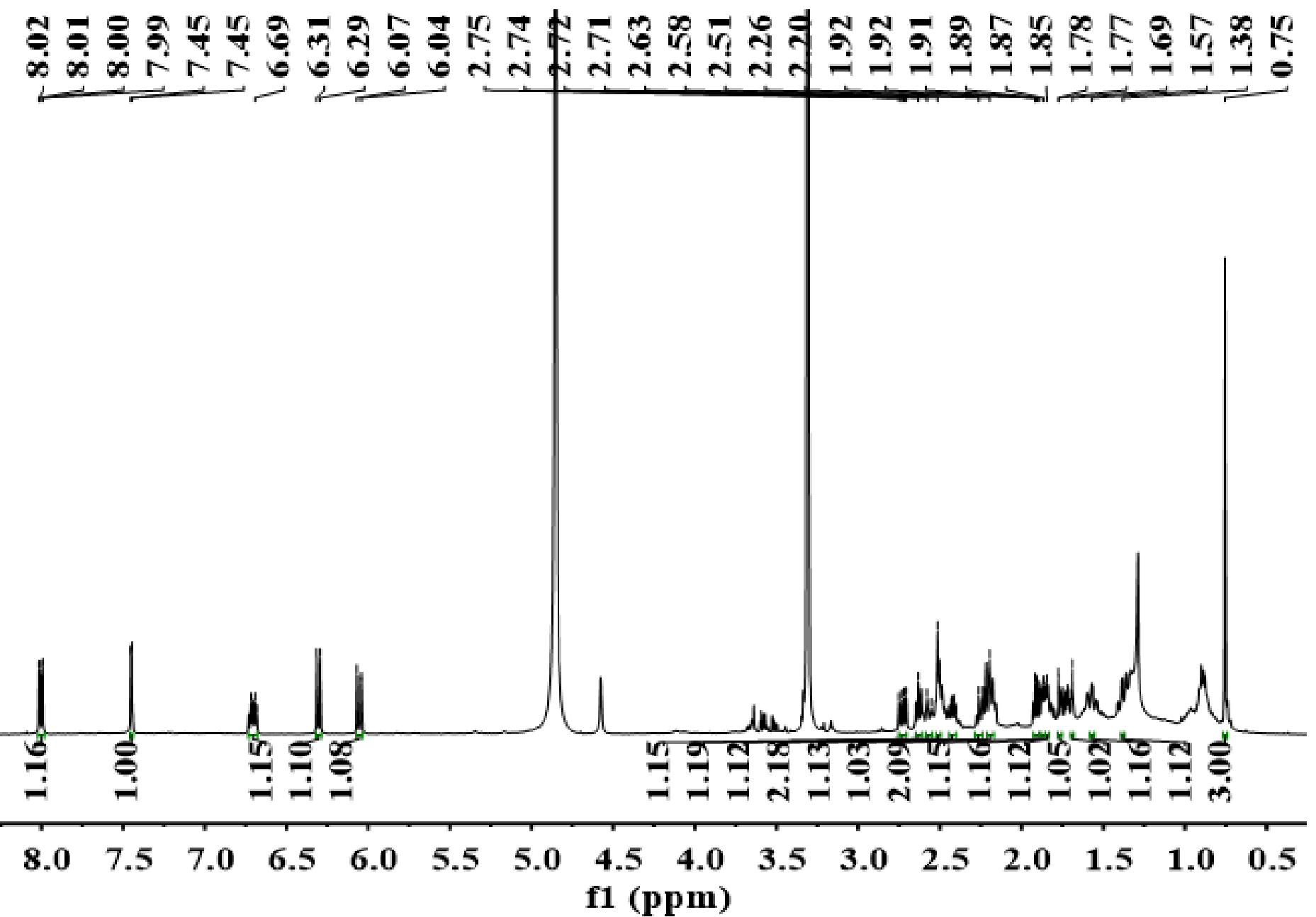

Figure S5. The ${ }^{1} \mathrm{H}-\mathrm{NMR}$ spectrum of compound $\mathbf{1}$ (in $\mathrm{CD}_{3} \mathrm{OD}, 500 \mathrm{MHz}$ ). 


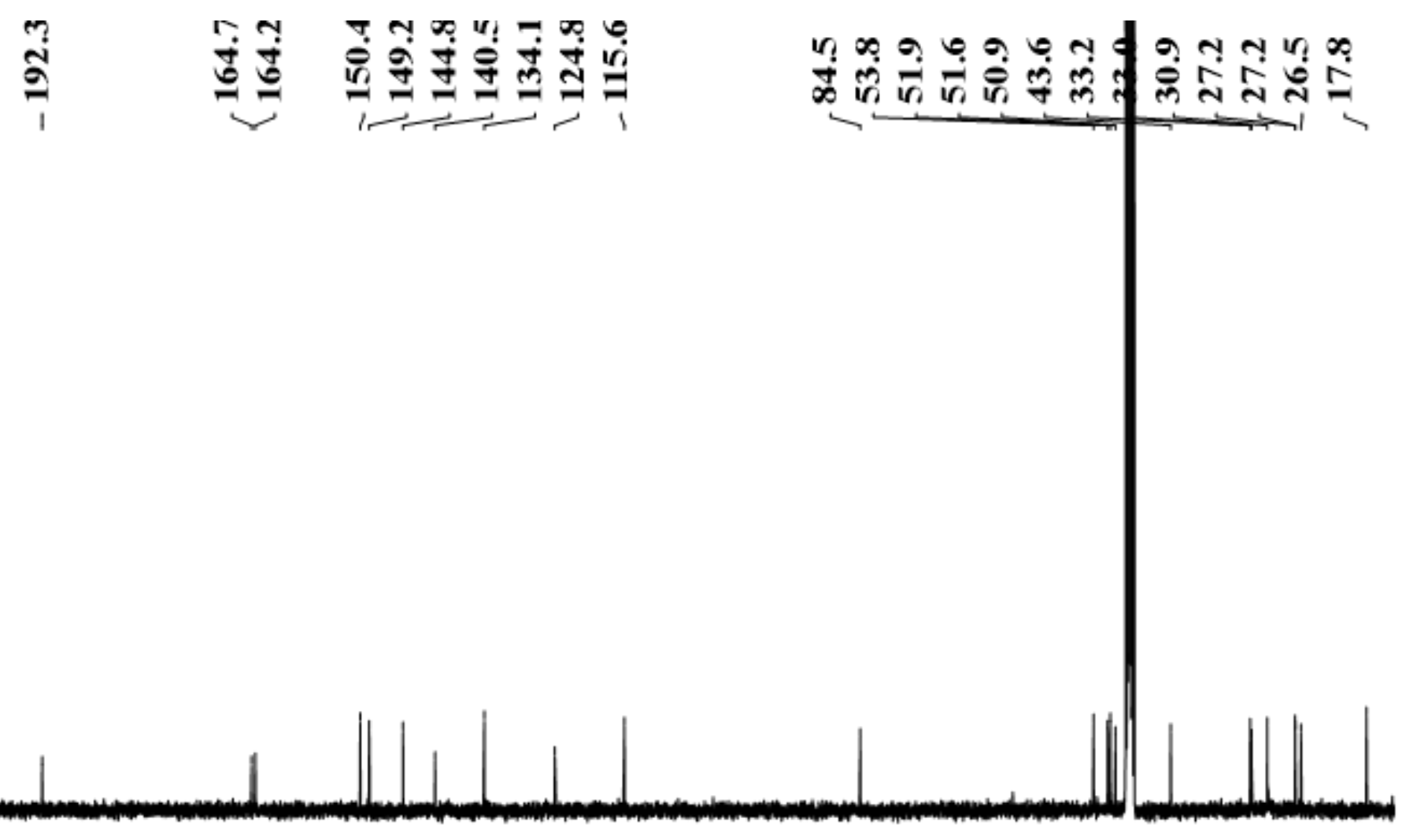

$\begin{array}{llllllllllllllllllll}190 & 180 & 170 & 160 & 150 & 140 & 130 & 120 & 110 & 100 & 90 & 80 & 70 & 60 & 50 & 40 & 30 & 20\end{array}$ f1 (ppm)

Figure S6. The ${ }^{13} \mathrm{C}-\mathrm{NMR}$ spectrum of compound 1 (in $\mathrm{CD}_{3} \mathrm{OD}, 125 \mathrm{MHz}$ ). 


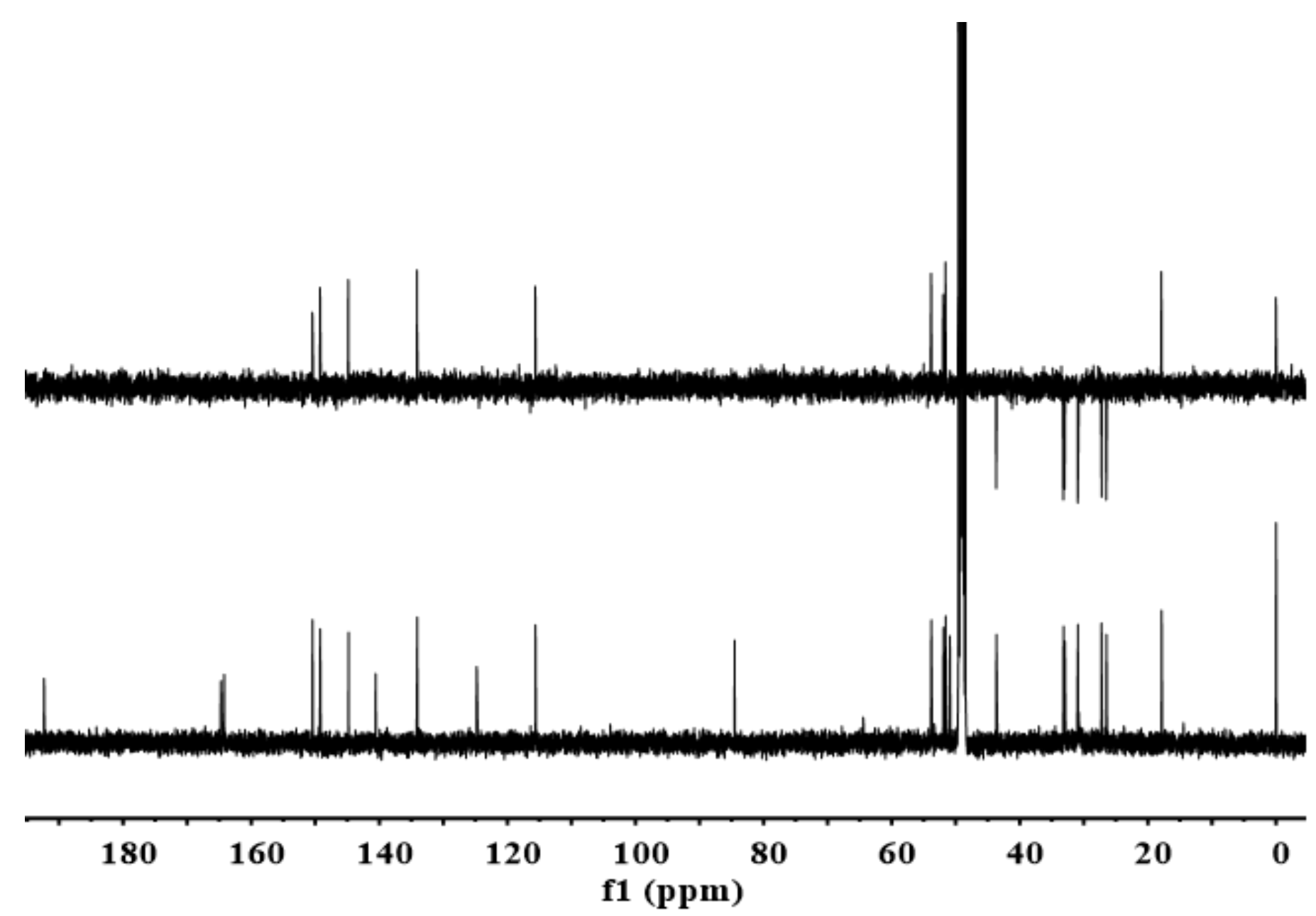

Figure S7. The ${ }^{13} \mathrm{C}-\mathrm{NMR}$ and DEPT 135 spectra of compound $\mathbf{1}$ (in $\mathrm{CD}_{3} \mathrm{OD}$ ). 


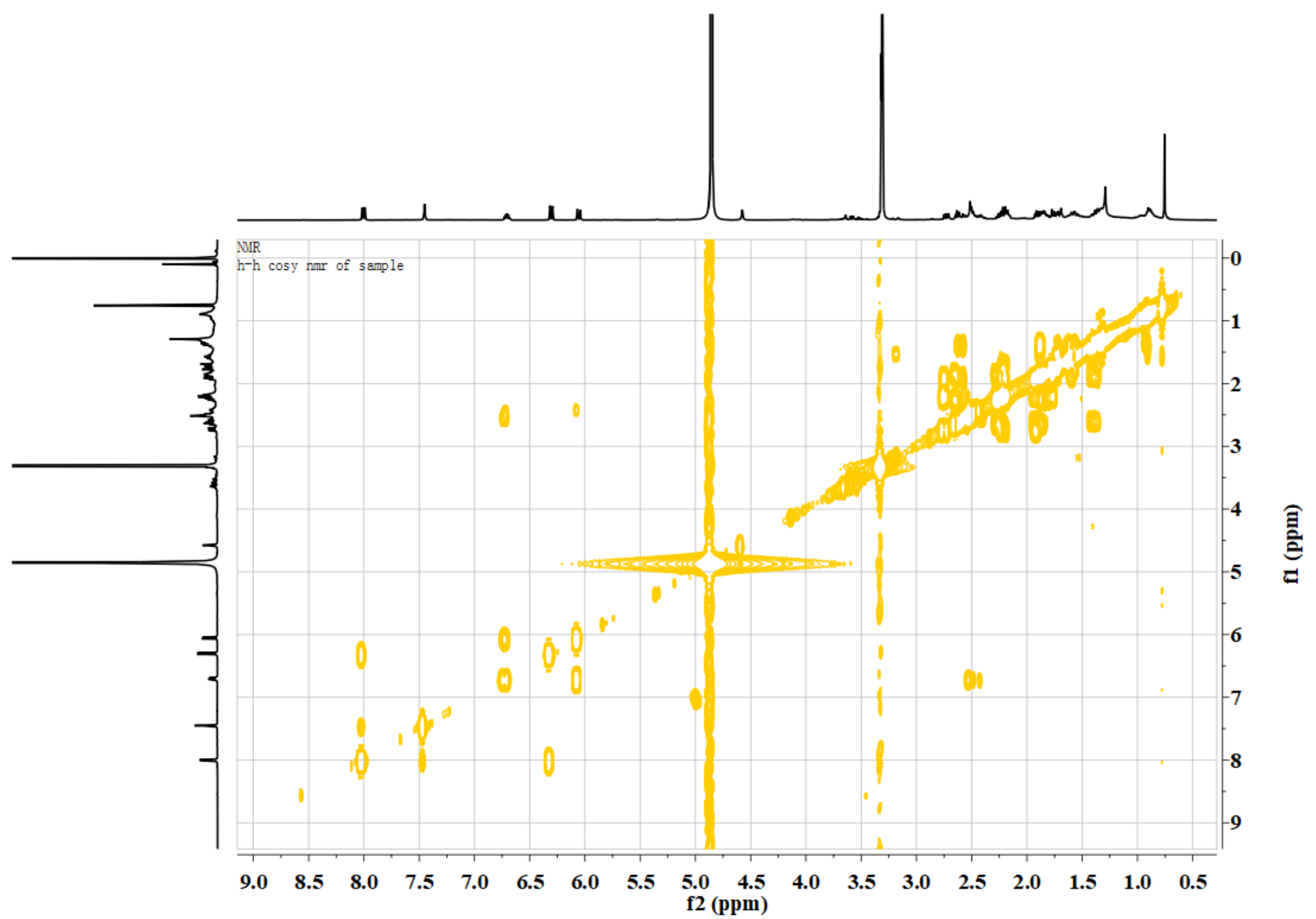

Figure S8. The ${ }^{1} \mathrm{H}_{-}{ }^{1} \mathrm{H}$ COSY spectrum of compound $\mathbf{1}$ (in $\mathrm{CD}_{3} \mathrm{OD}$ ). 


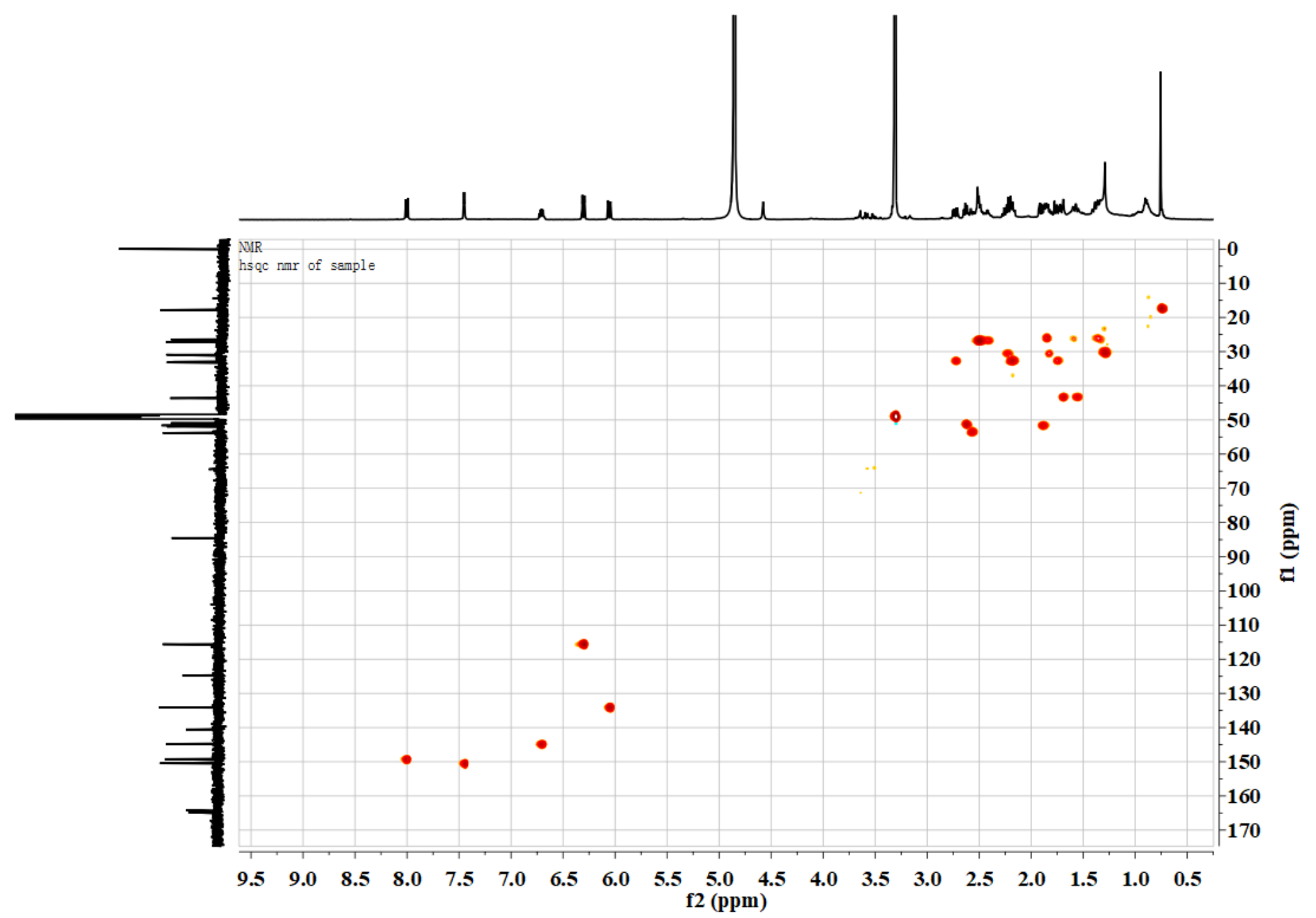

Figure S9. The HSQC spectrum of compound 1 (in $\mathrm{CD}_{3} \mathrm{OD}$ ). 


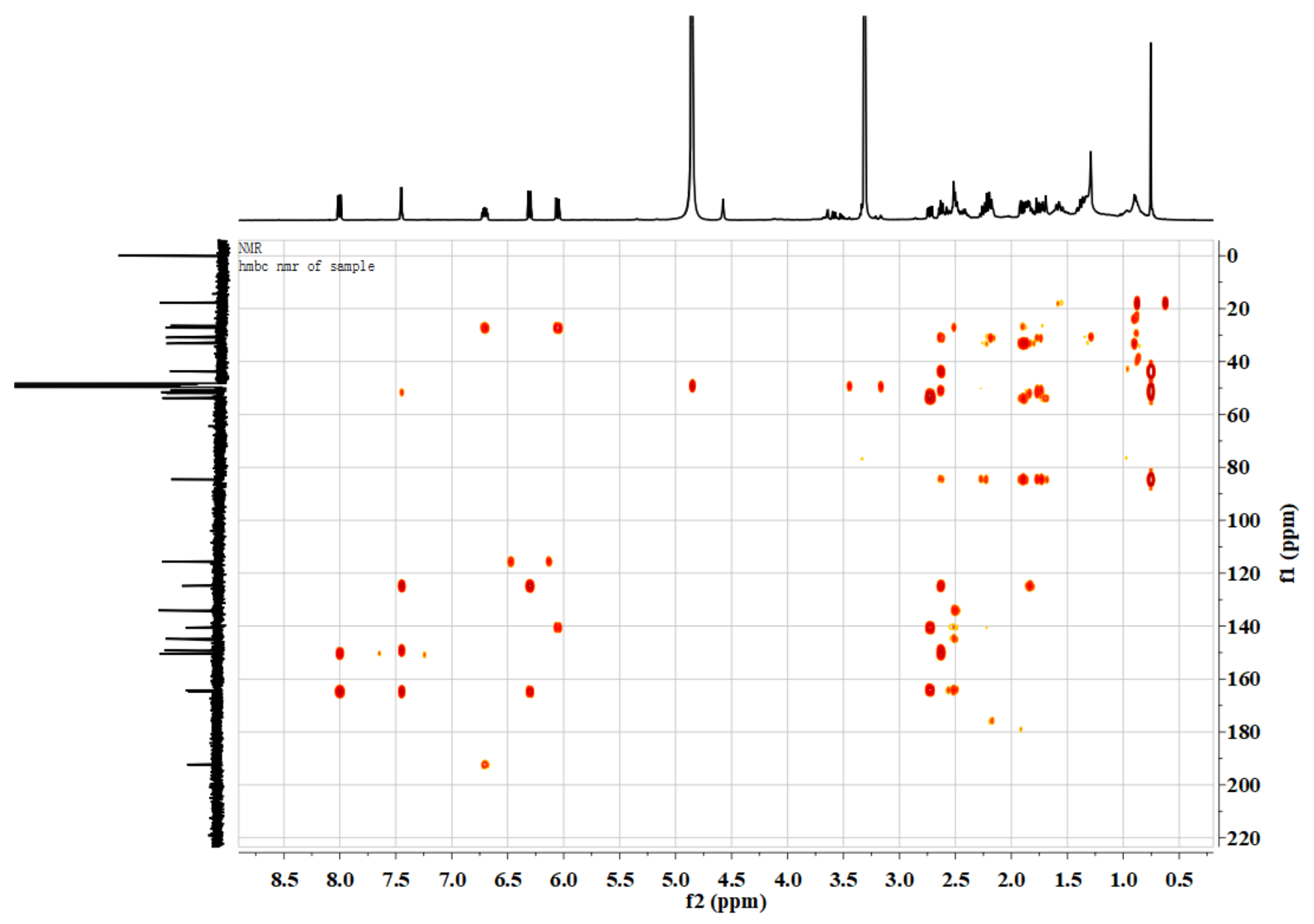

Figure S10. The HMBC spectrum of compound 1 (in $\mathrm{CD}_{3} \mathrm{OD}$ ). 


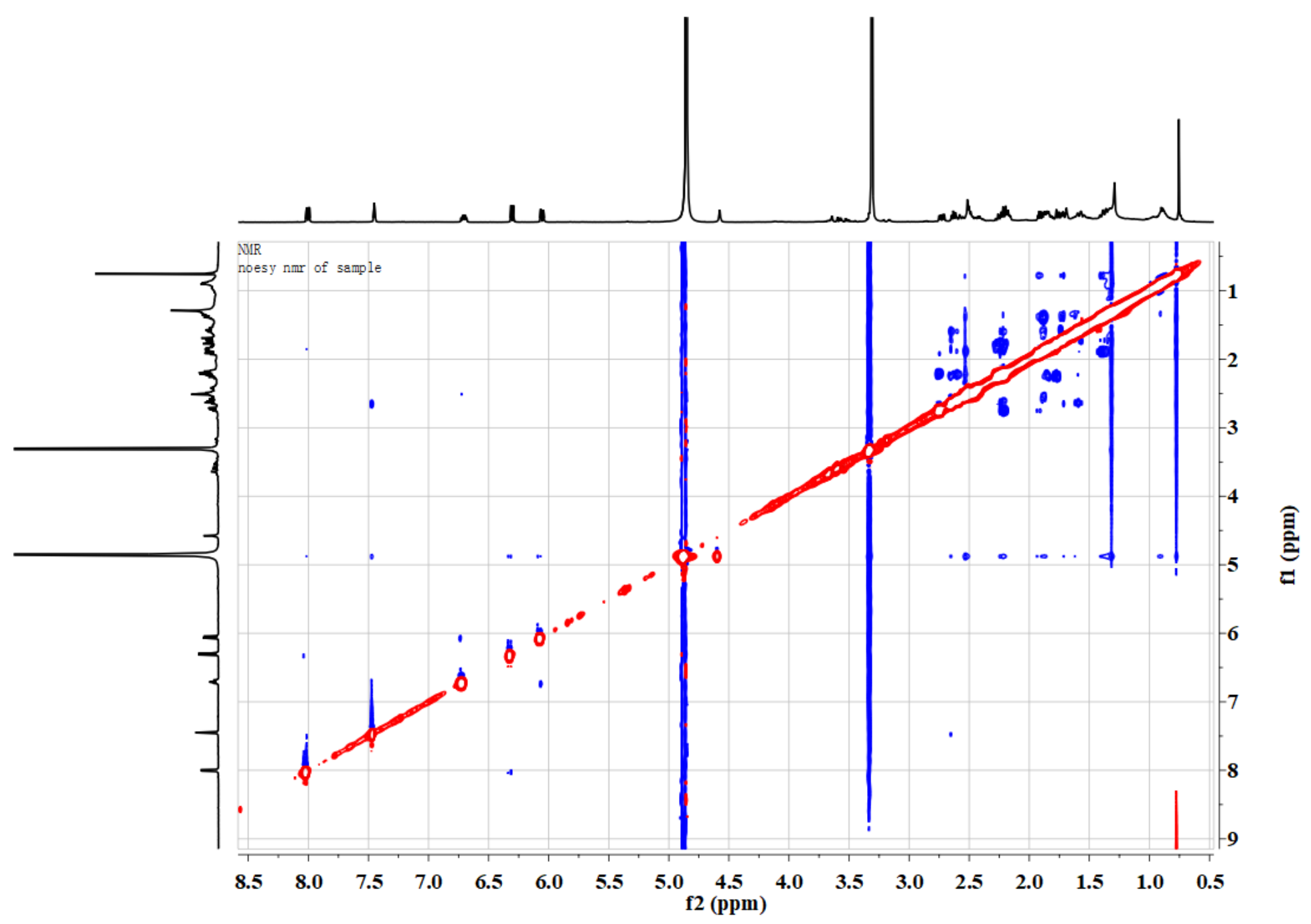

Figure S11. The NOESY spectrum of Compound 1 (in $\mathrm{CD}_{3} \mathrm{OD}$ ). 


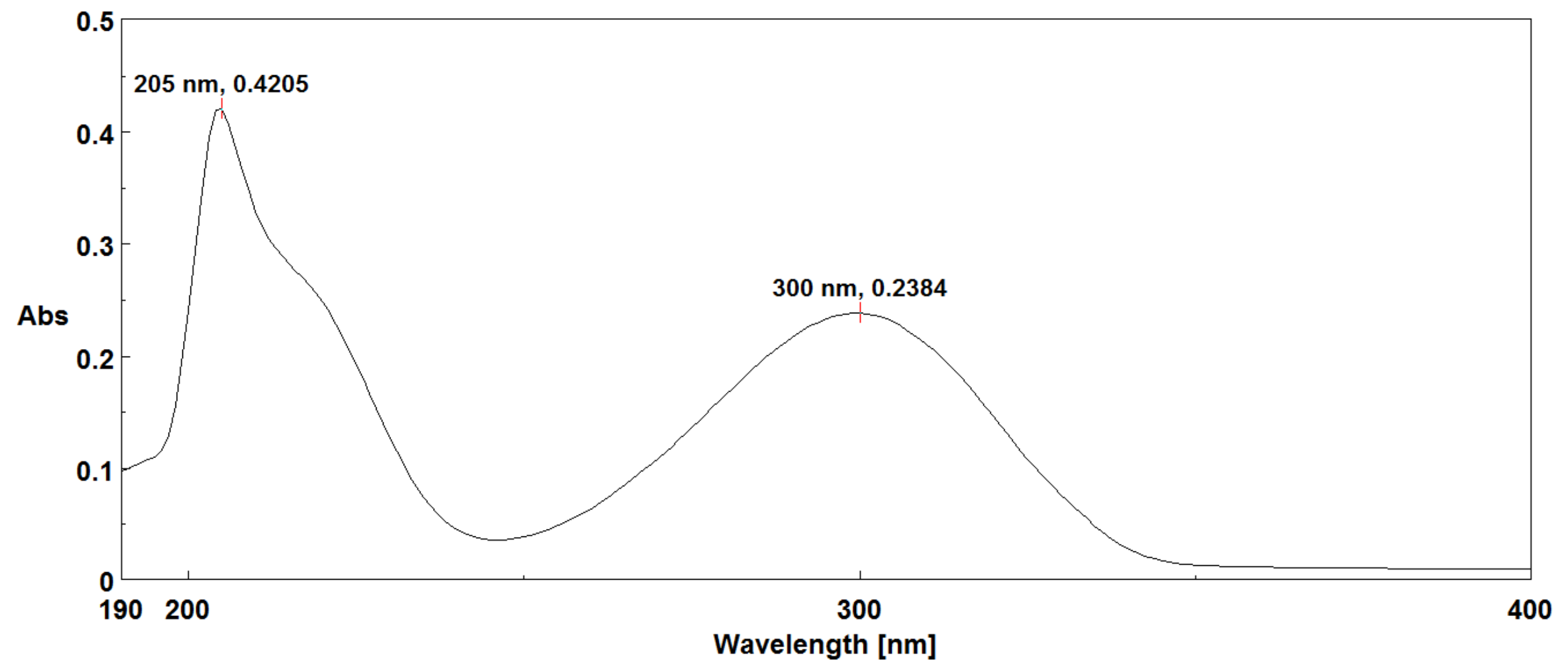

Figure S12. The UV spectrum of compound 2 (in $\mathrm{MeOH}$ ). 


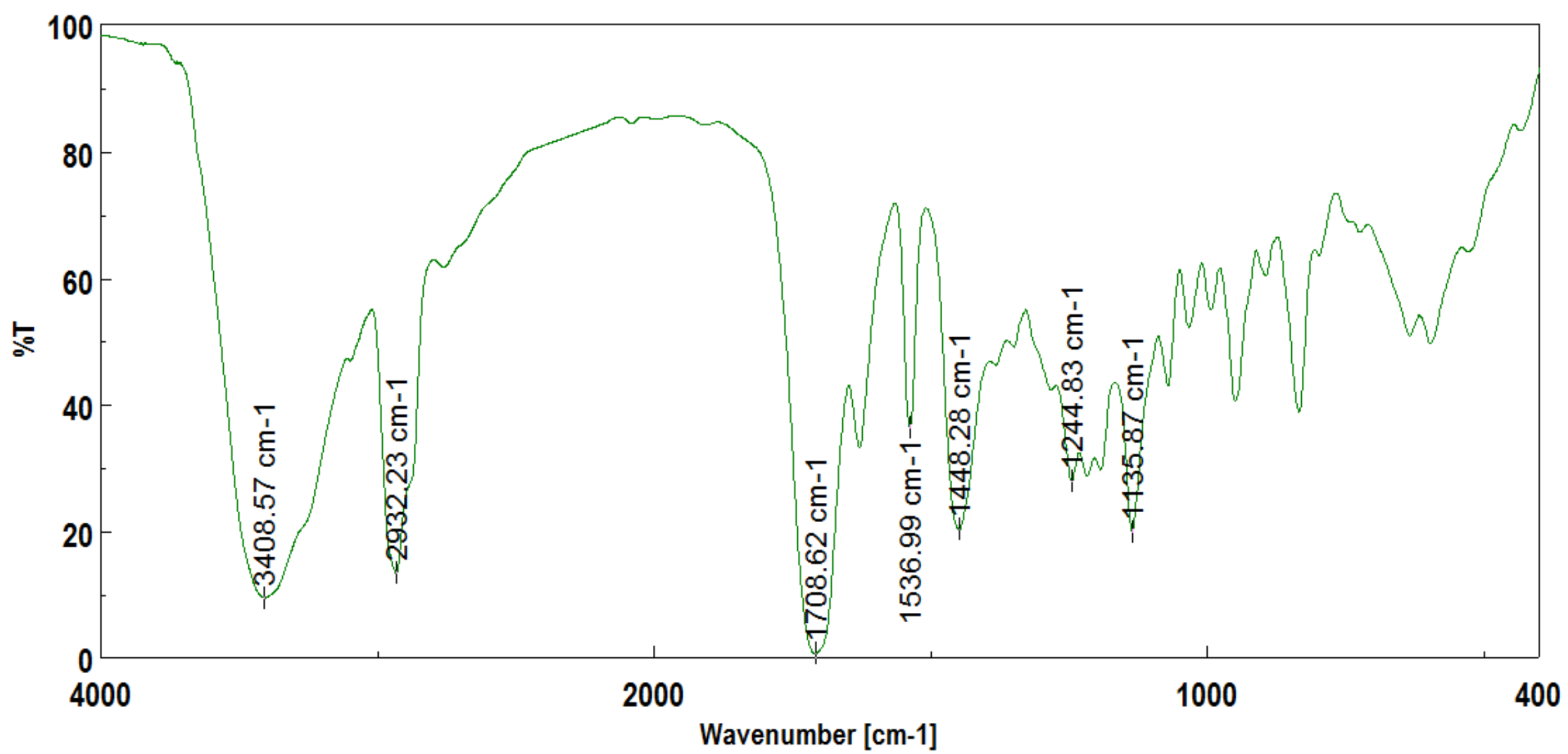

Figure S13. The IR spectrum of compound 2 (in $\mathrm{KBr}$ ). 


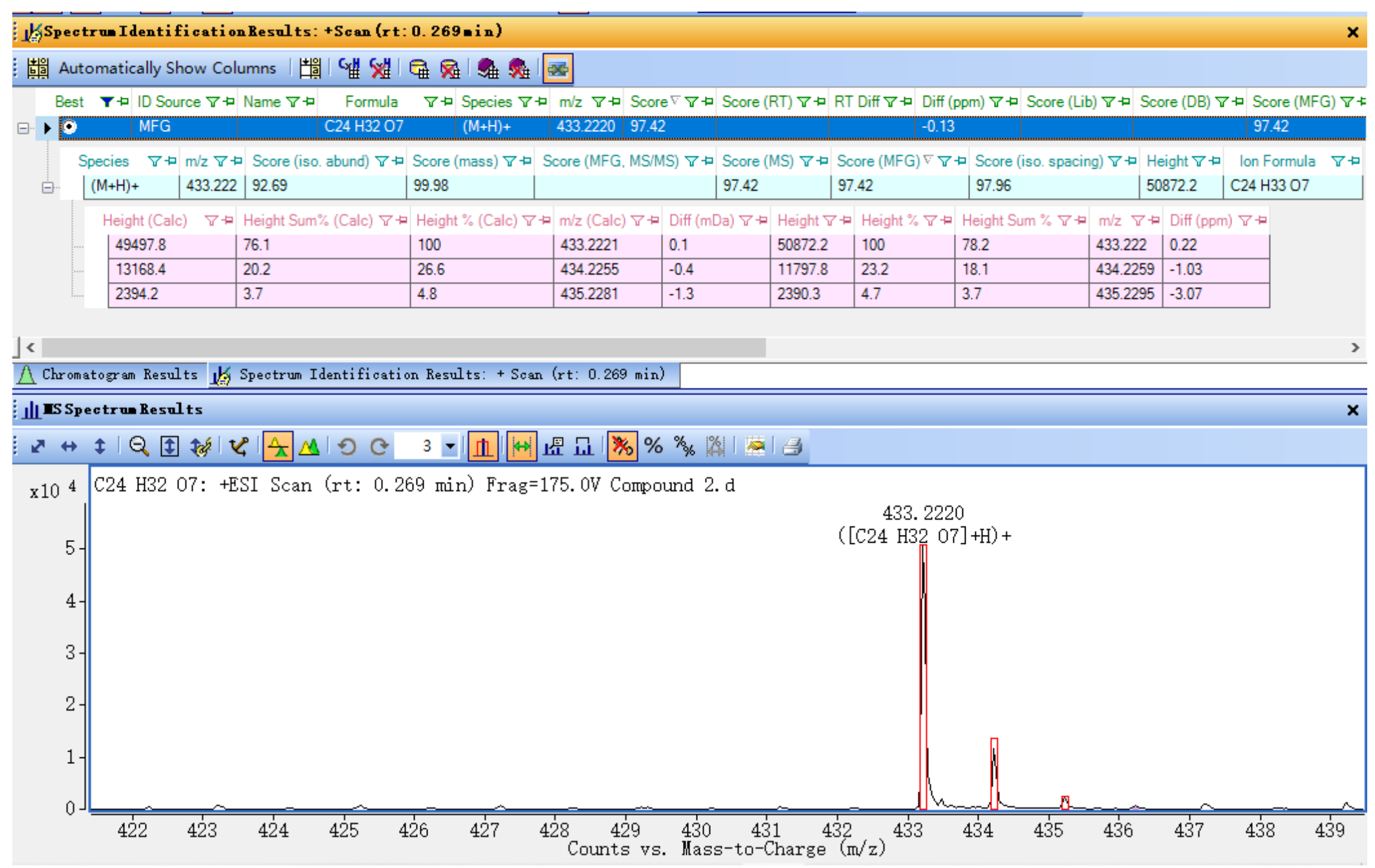

Figure S14. The HRESIMS spectrum of compound 2. 
$\underbrace{\infty}$

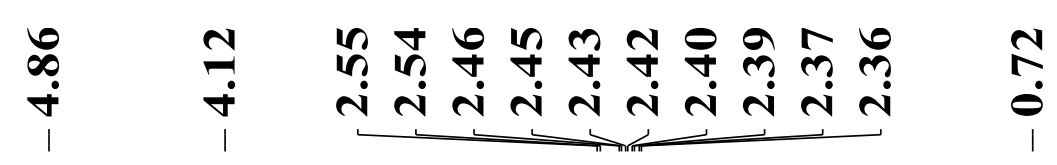

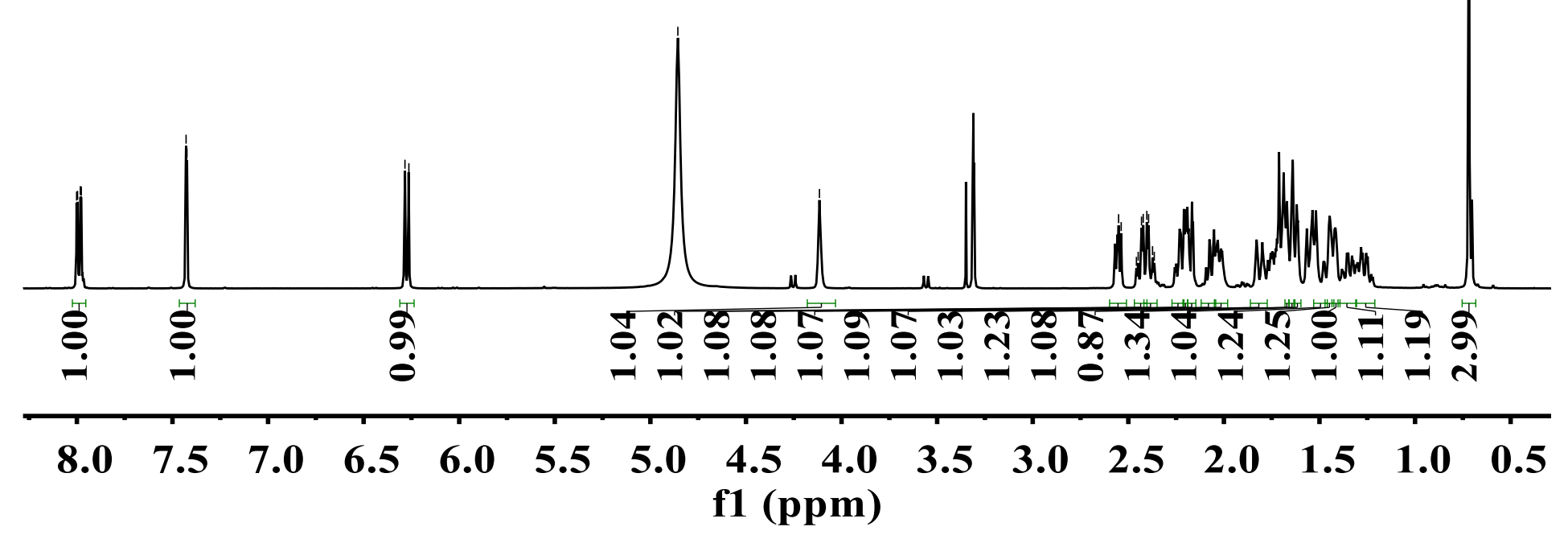

Figure S15. The ${ }^{1} \mathrm{H}-\mathrm{NMR}$ spectrum of compound 2 (in $\mathrm{CD}_{3} \mathrm{OD}, 500 \mathrm{MHz}$ ).

27 

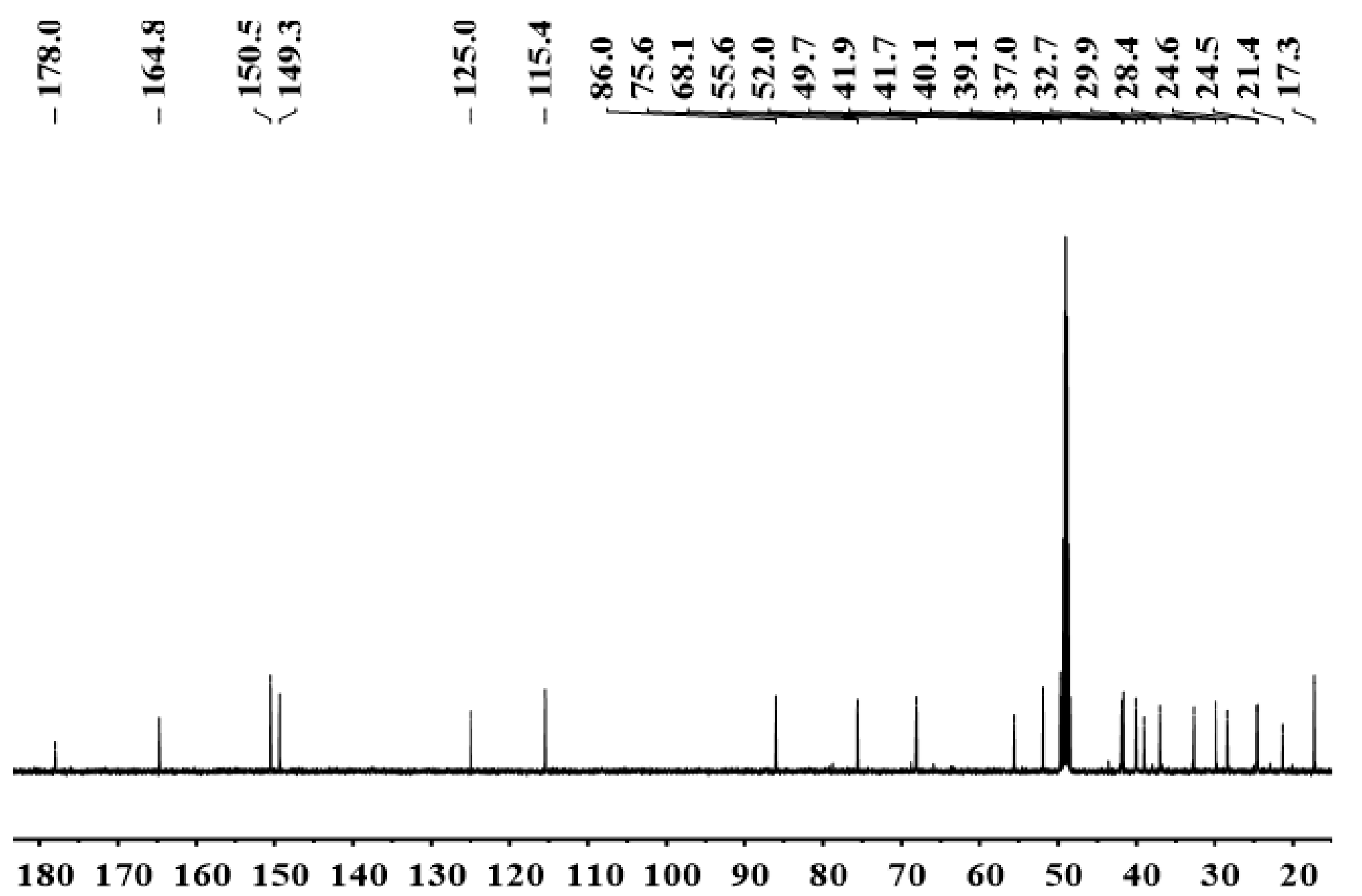

f1 (ppm)

Figure S16. The ${ }^{13} \mathrm{C}-\mathrm{NMR}$ spectrum of compound 2 (in $\mathrm{CD}_{3} \mathrm{OD}, 125 \mathrm{MHz}$ ). 


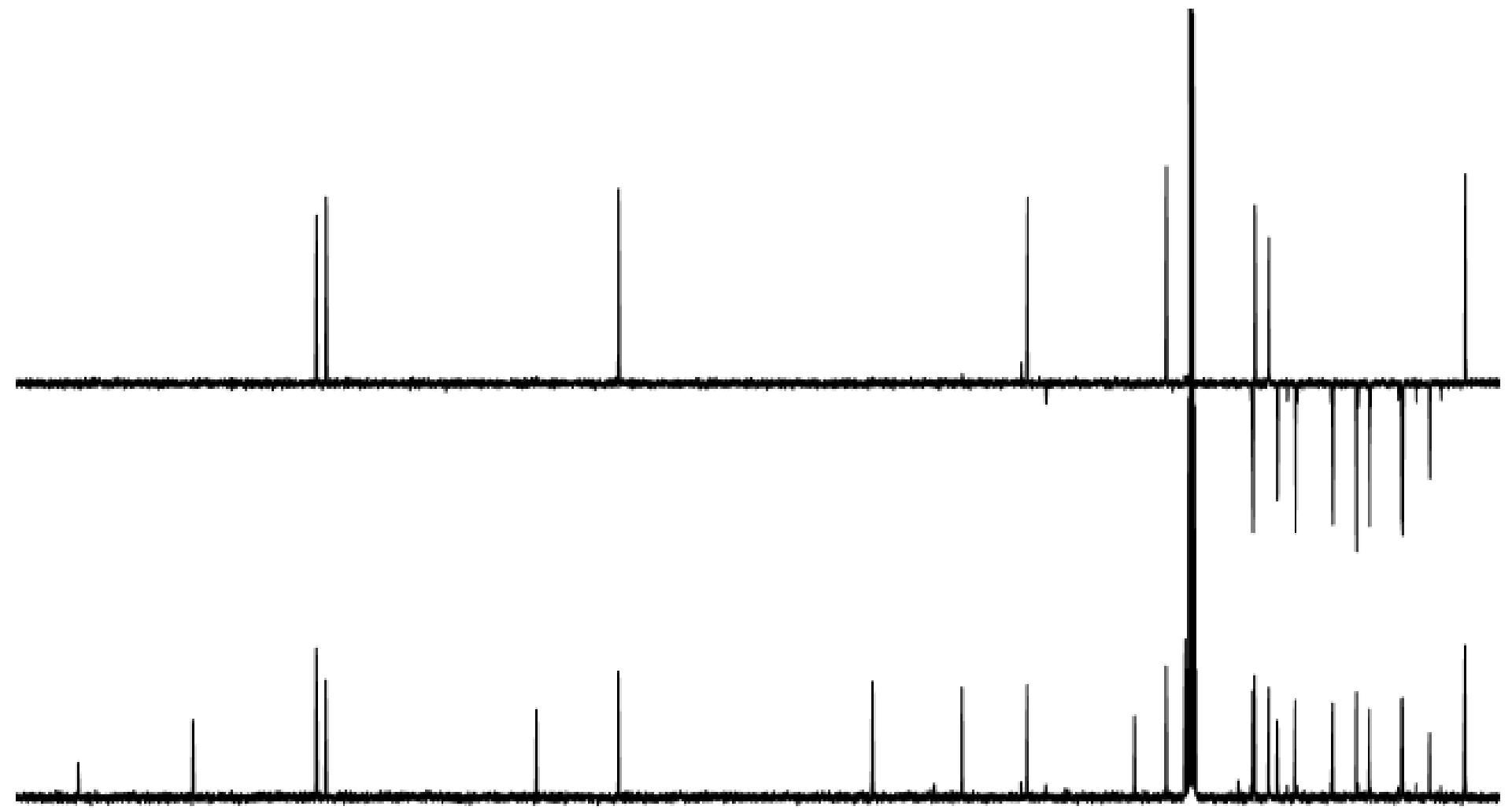

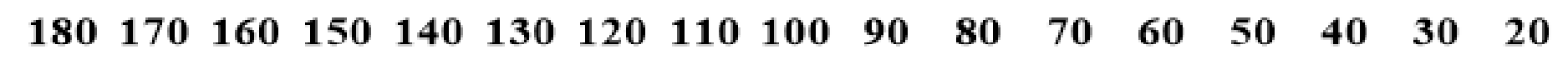
f1 (ppm)

Figure S17. The ${ }^{13} \mathrm{C}-\mathrm{NMR}$ and DEPT 135 spectra of compound 2 (in $\mathrm{CD}_{3} \mathrm{OD}$ ). 


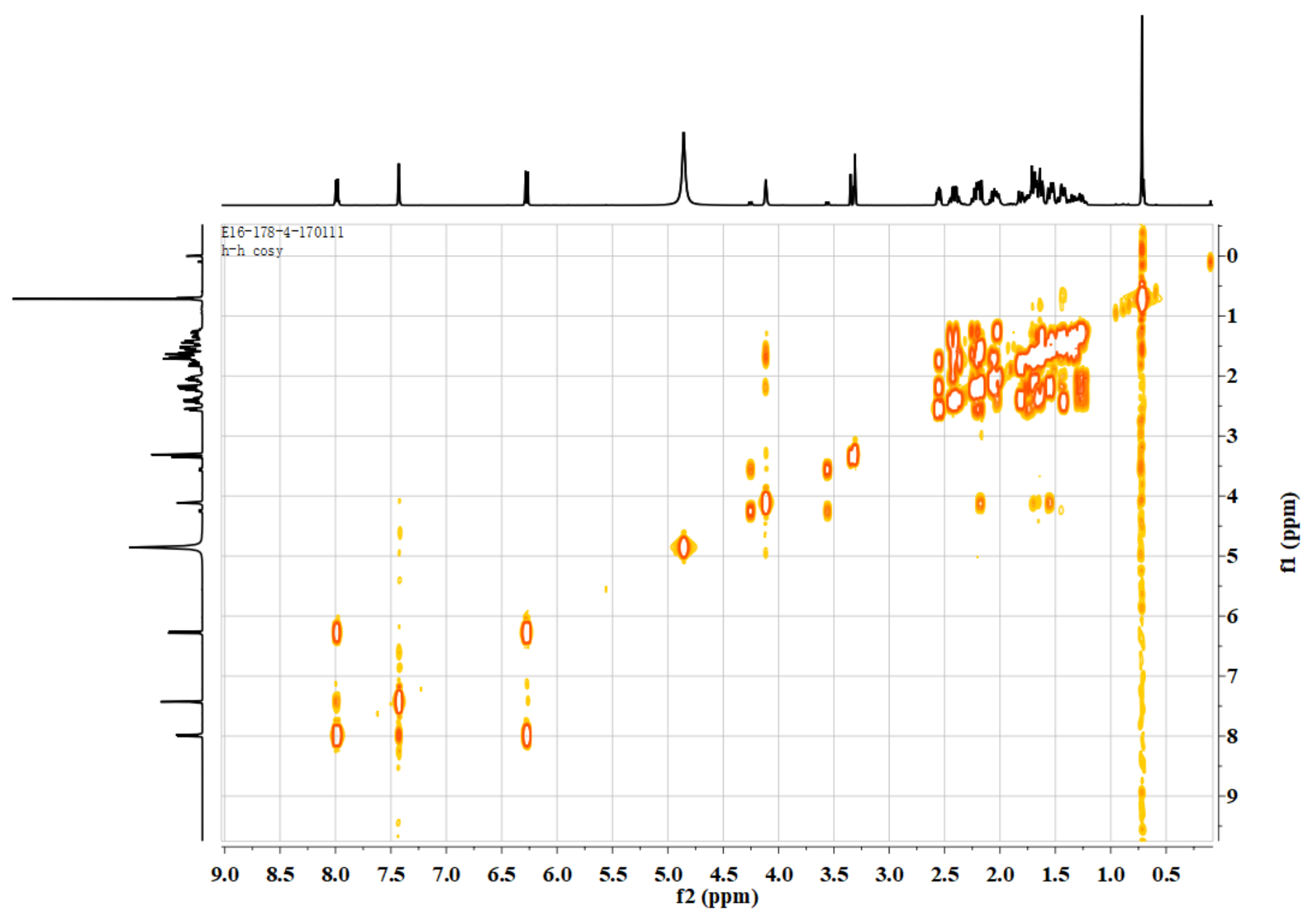

Figure S18. The ${ }^{1} \mathrm{H}-{ }^{1} \mathrm{H}$ COSY spectrum of compound 2 (in $\mathrm{CD}_{3} \mathrm{OD}$ ). 


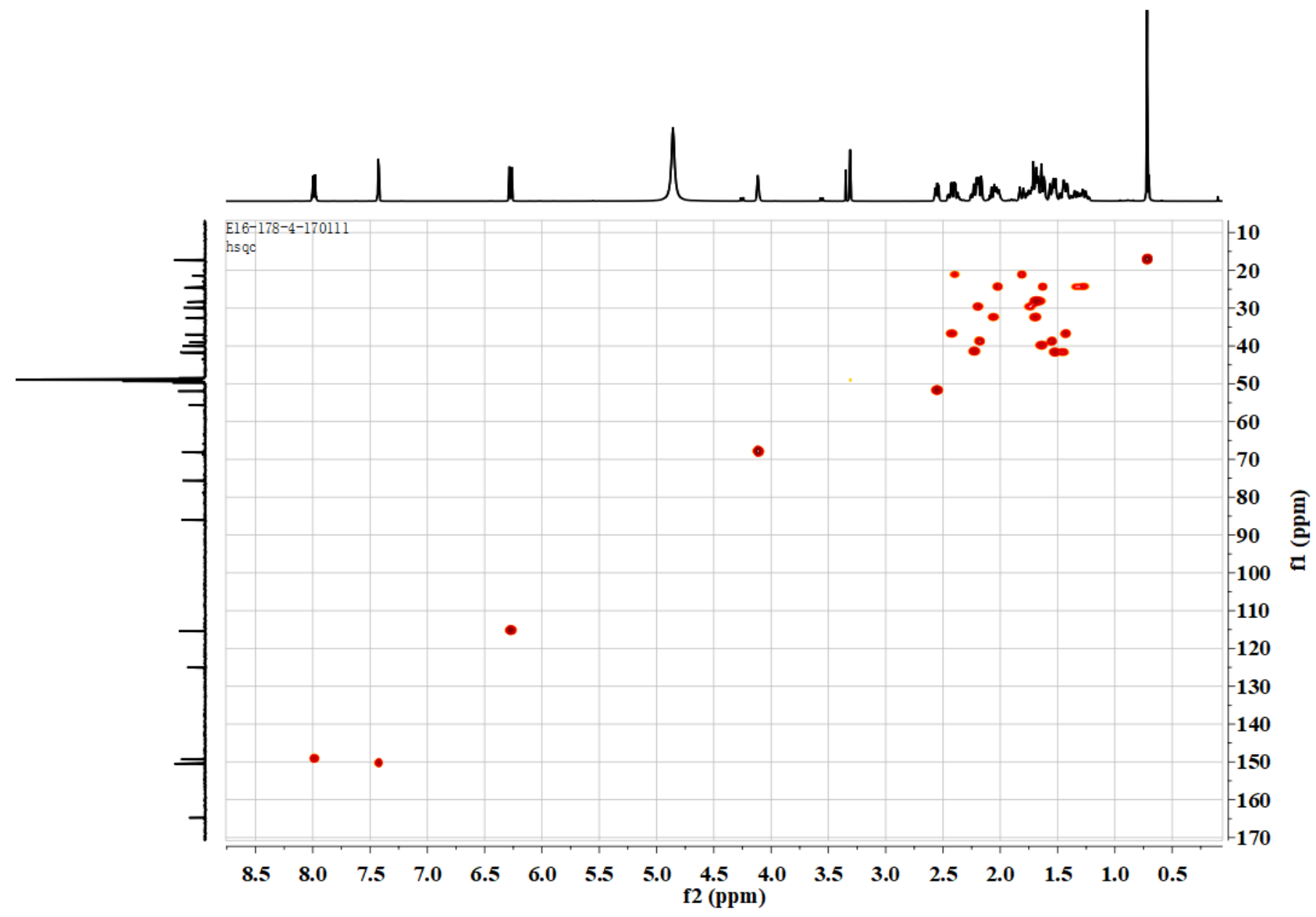

Figure S19. The HSQC spectrum of compound 2 (in $\mathrm{CD}_{3} \mathrm{OD}$ ). 


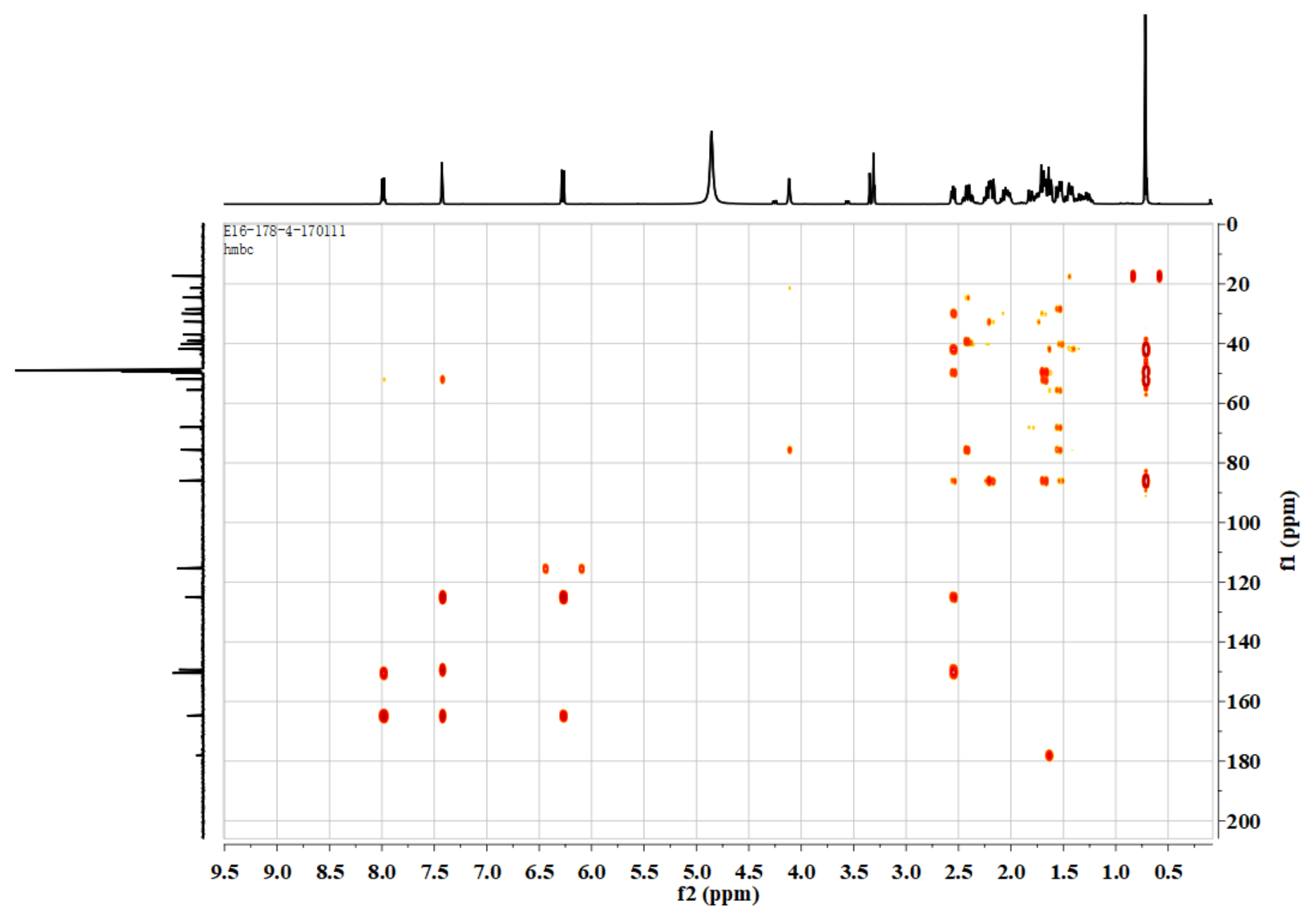

Figure S20. The HMBC spectrum of compound 2 (in $\mathrm{CD}_{3} \mathrm{OD}$ ). 


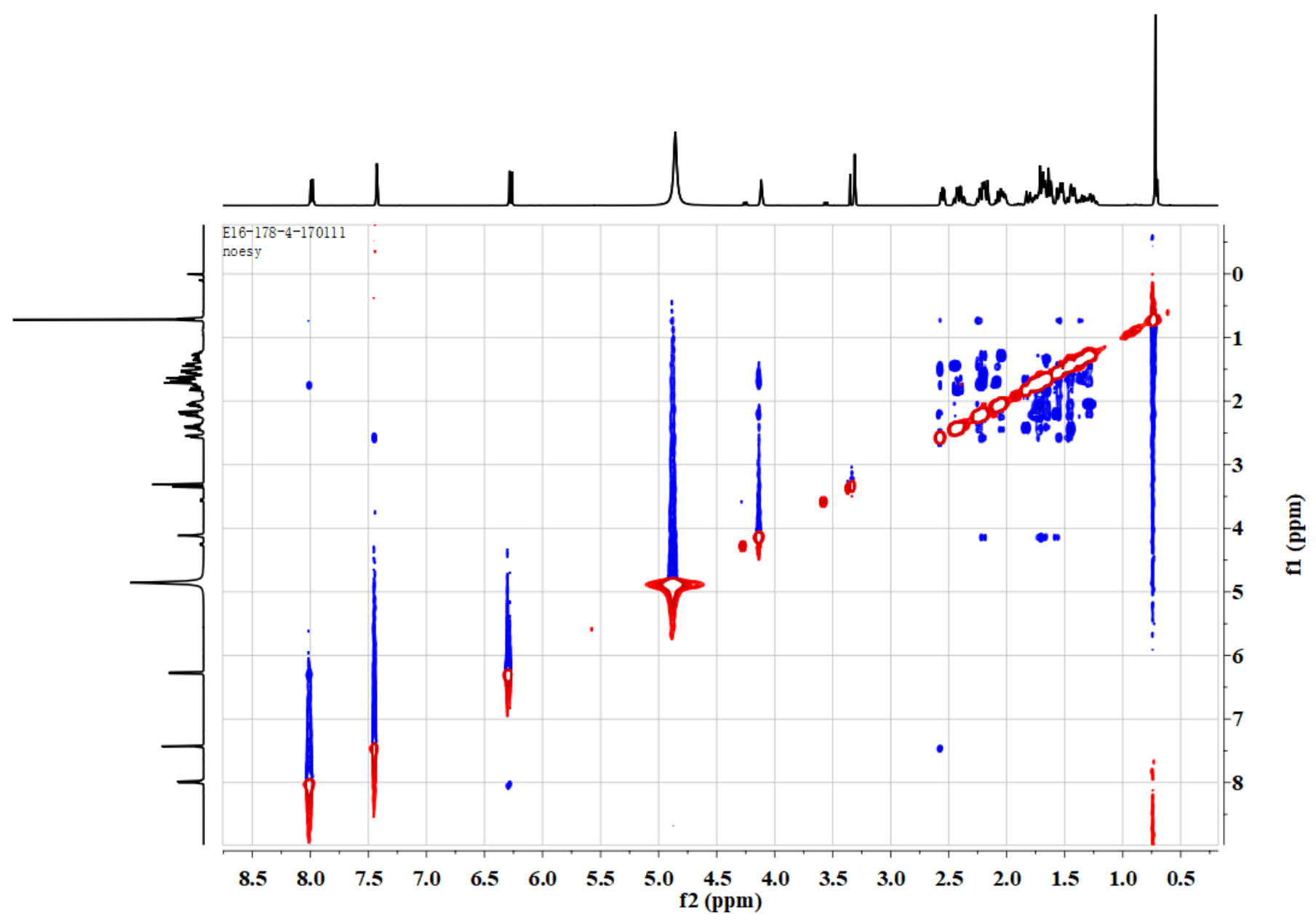

Figure S21. The NOESY spectrum of compound 2 (in CD3OD). 


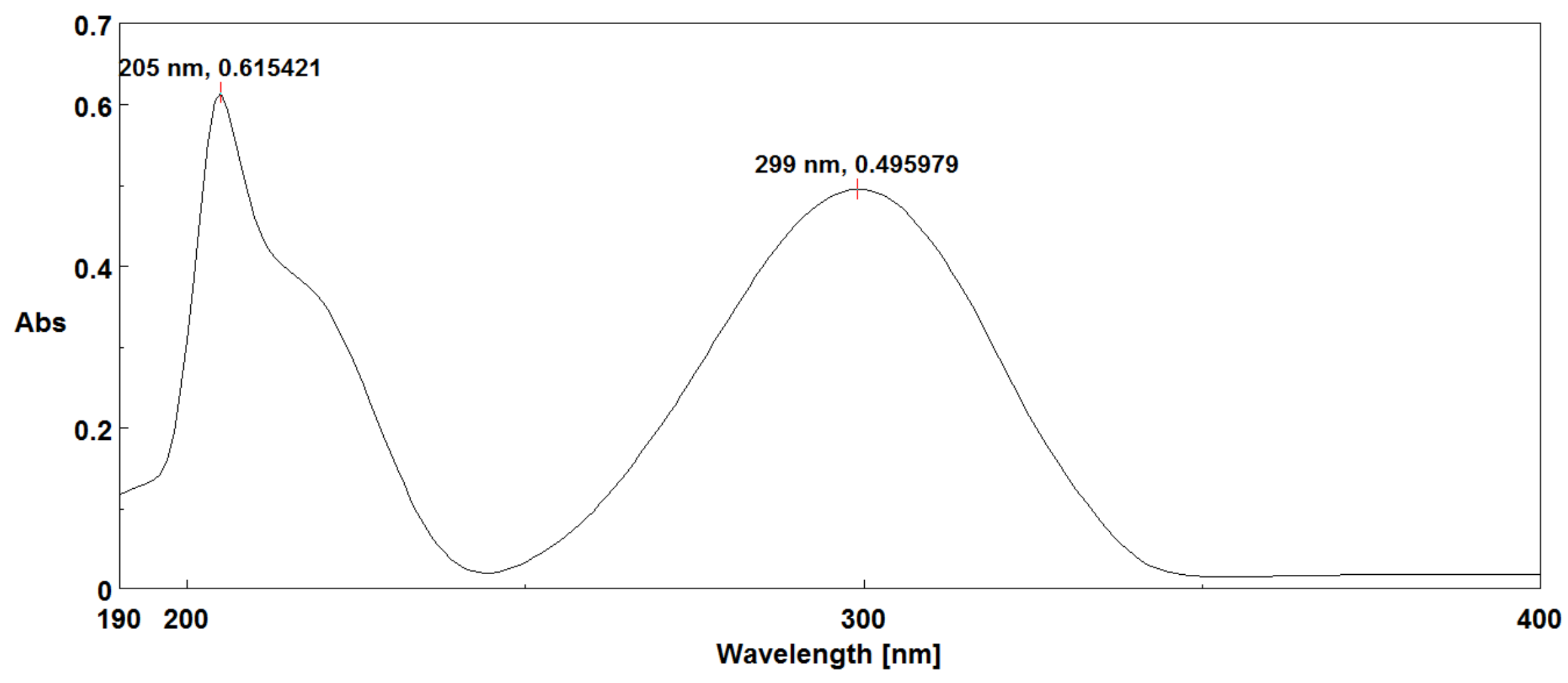

Figure S22. The UV spectrum of compound 3 (in $\mathrm{MeOH}$ ). 


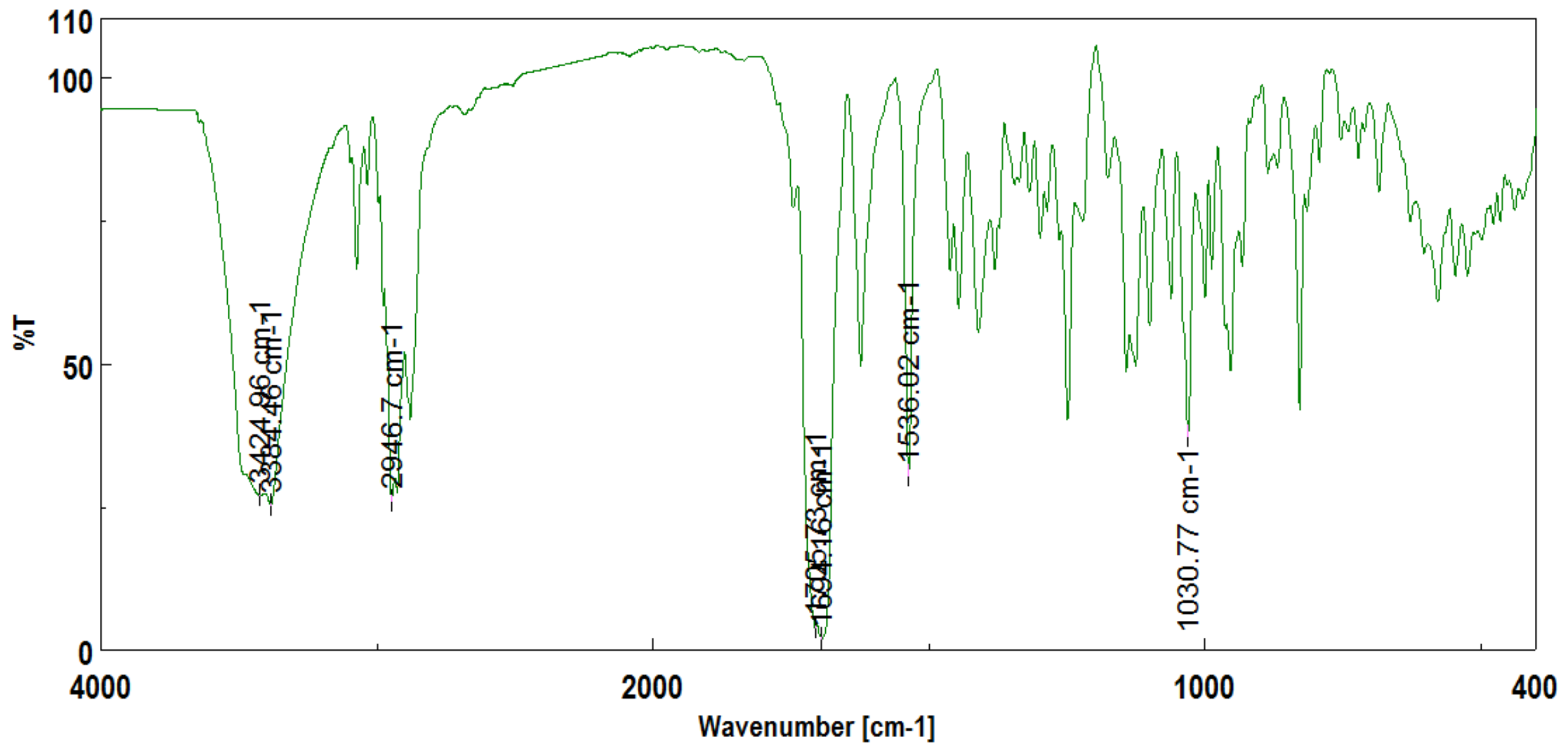

Figure S23. The IR spectrum of compound $\mathbf{3}$ (in $\mathrm{KBr}$ ). 


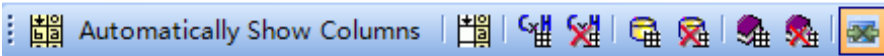

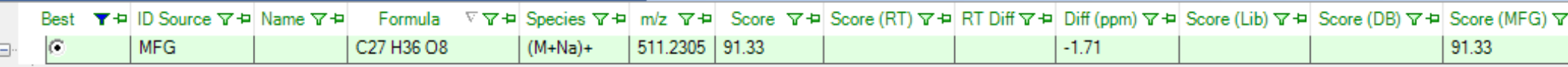

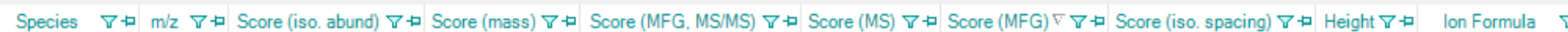
\begin{tabular}{|l|l|l|l|l|l|l|l|l}
$(\mathrm{M}+\mathrm{Na})+$ & 511.2305 & 83.71 & 97.09 & 91.33 & 91.33 & 88.95 & 12124 & $\mathrm{C} 27 \mathrm{H} 36 \mathrm{Na} 08$ \\
\hline
\end{tabular}

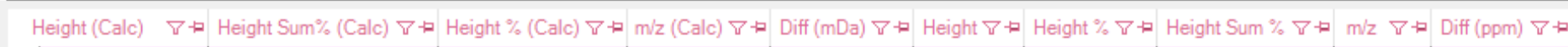

\begin{tabular}{|l|l|l|l|l|l|l|l|l|l|}
12615 & 73.1 & 100 & 511.2302 & -0.3 & 12124 & 100 & 70.3 & 511.2305 & -0.58 \\
\hline 3774.6 & 21.9 & 29.9 & 512.2336 & -1.2 & 3640.9 & 30 & 21.1 & 512.2348 & -2.33 \\
\hline 752.2 & 4.4 & 6 & 513.2363 & -2.8 & 1270.4 & 10.5 & 7.4 & 513.2391 & -5.39 \\
\hline 112.5 & 0.7 & 0.9 & 514.239 & -13.7 & 218.9 & 1.8 & 1.3 & 514.2527 & -26.55 \\
\hline
\end{tabular}

\<

Chromatogram Results 1 Spectrum Identification Results: + Scan ( $r$ t: $0.347 \mathrm{~min})$

\section{illis Spectrue Results}

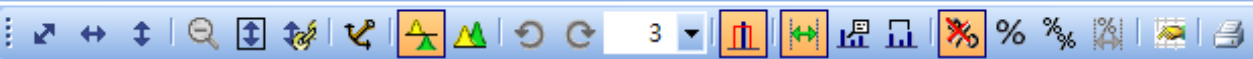

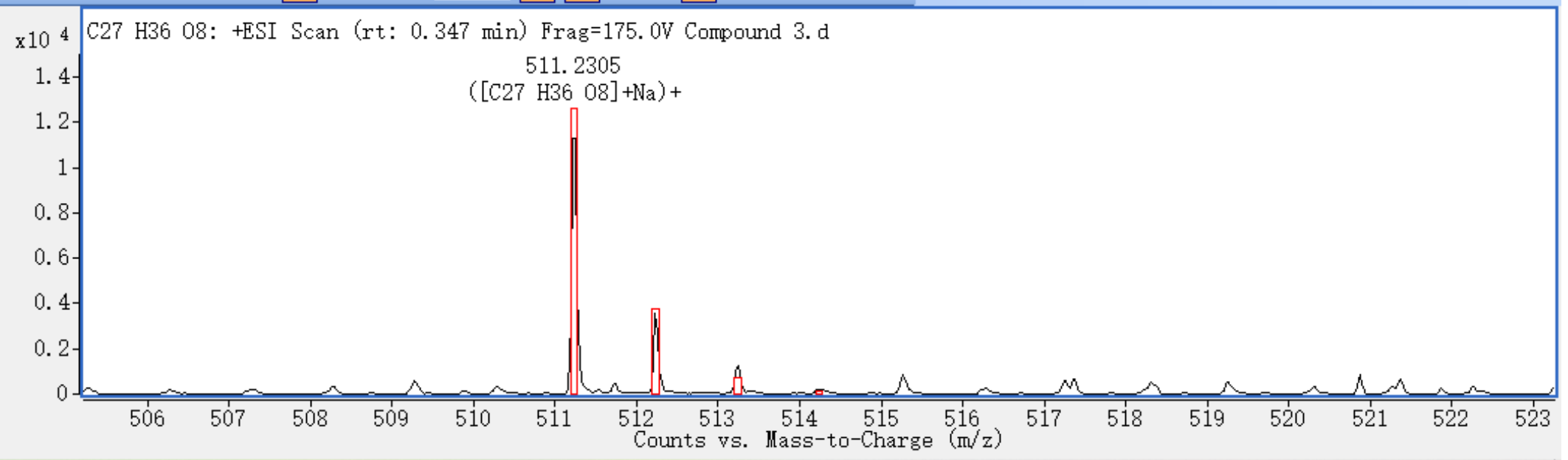

Figure S24. The HRESIMS spectrum of compound 3. 


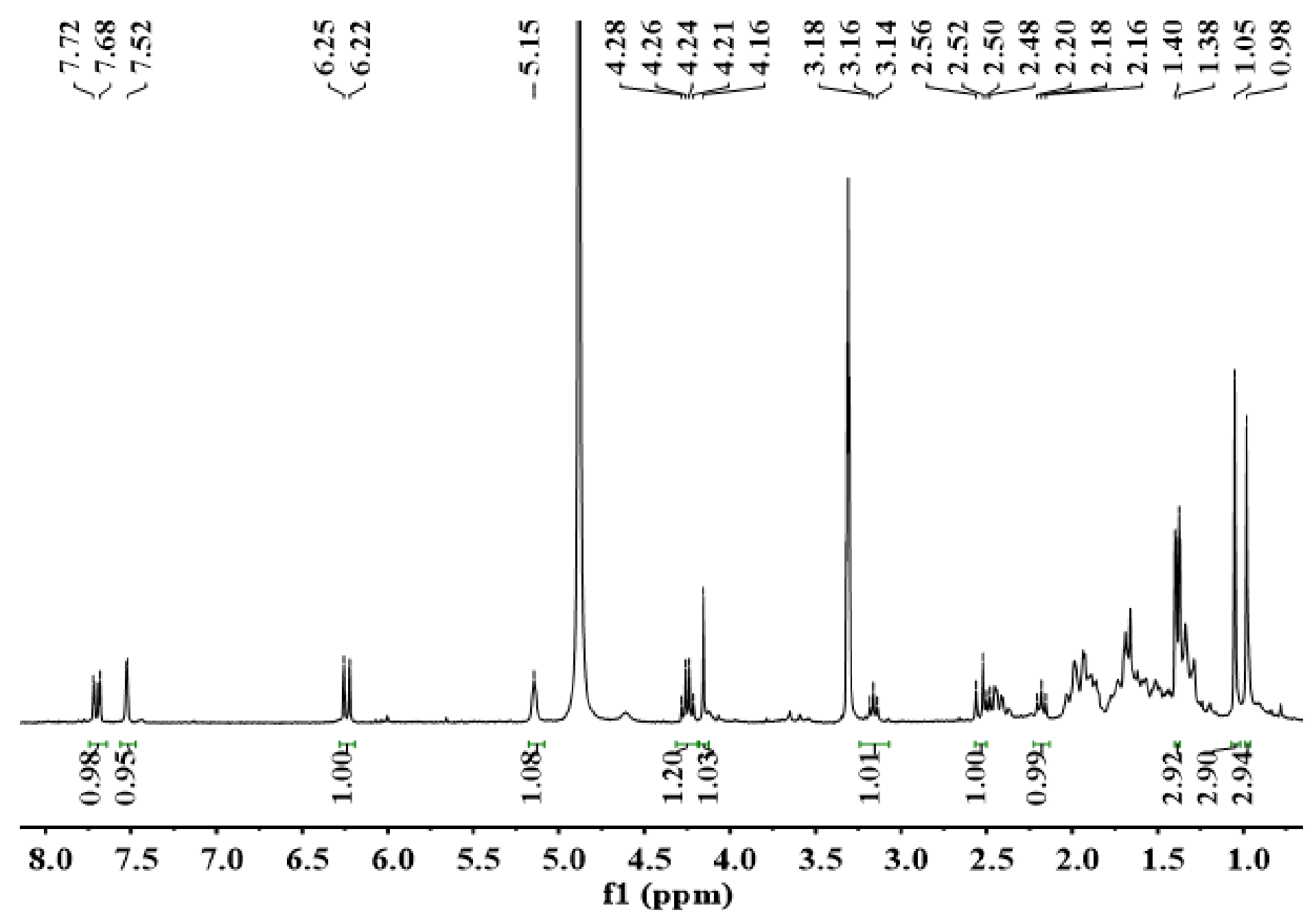

Figure S25. The ${ }^{1} \mathrm{H}-\mathrm{NMR}$ spectrum of compound 3 (in $\mathrm{CD}_{3} \mathrm{OD}, 400 \mathrm{MHz}$ ). 

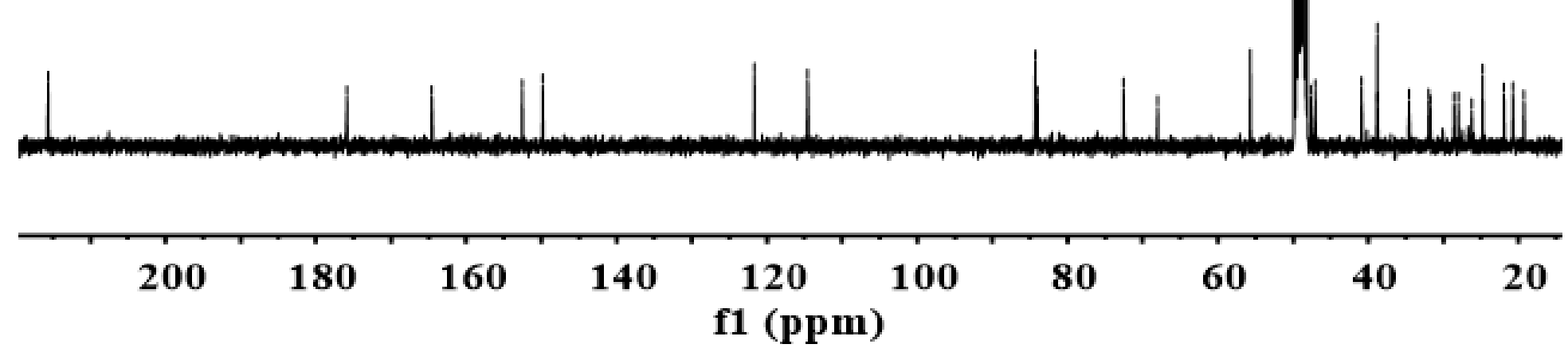

Figure S26. The ${ }^{13} \mathrm{C}$-NMR spectrum of compound 3 (in $\mathrm{CD}_{3} \mathrm{OD}, 100 \mathrm{MHz}$ ). 

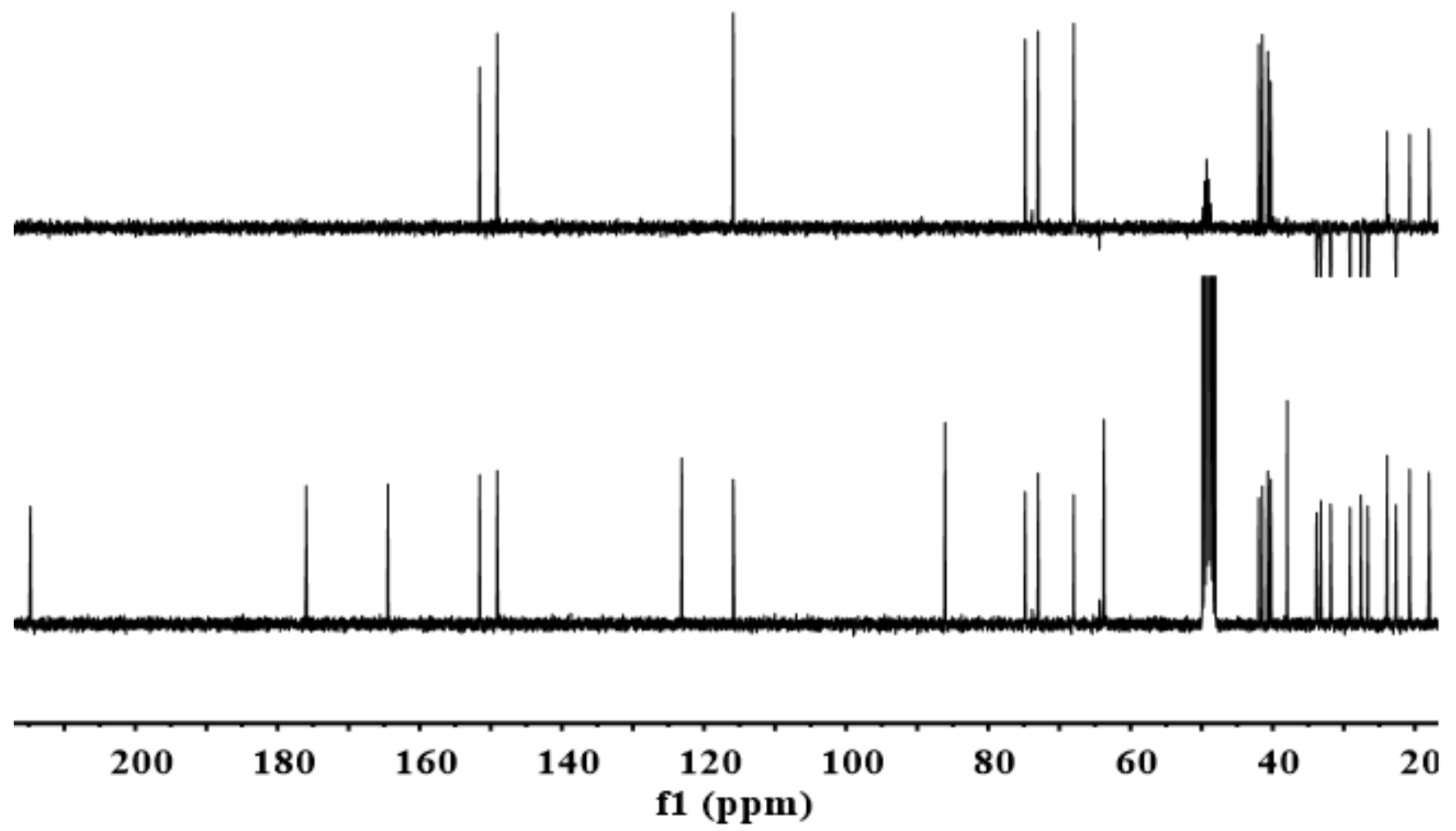

Figure S27. The ${ }^{13} \mathrm{C}-\mathrm{NMR}$ and DEPT 135 spectra of compound 3 (in $\mathrm{CD}_{3} \mathrm{OD}$ ). 


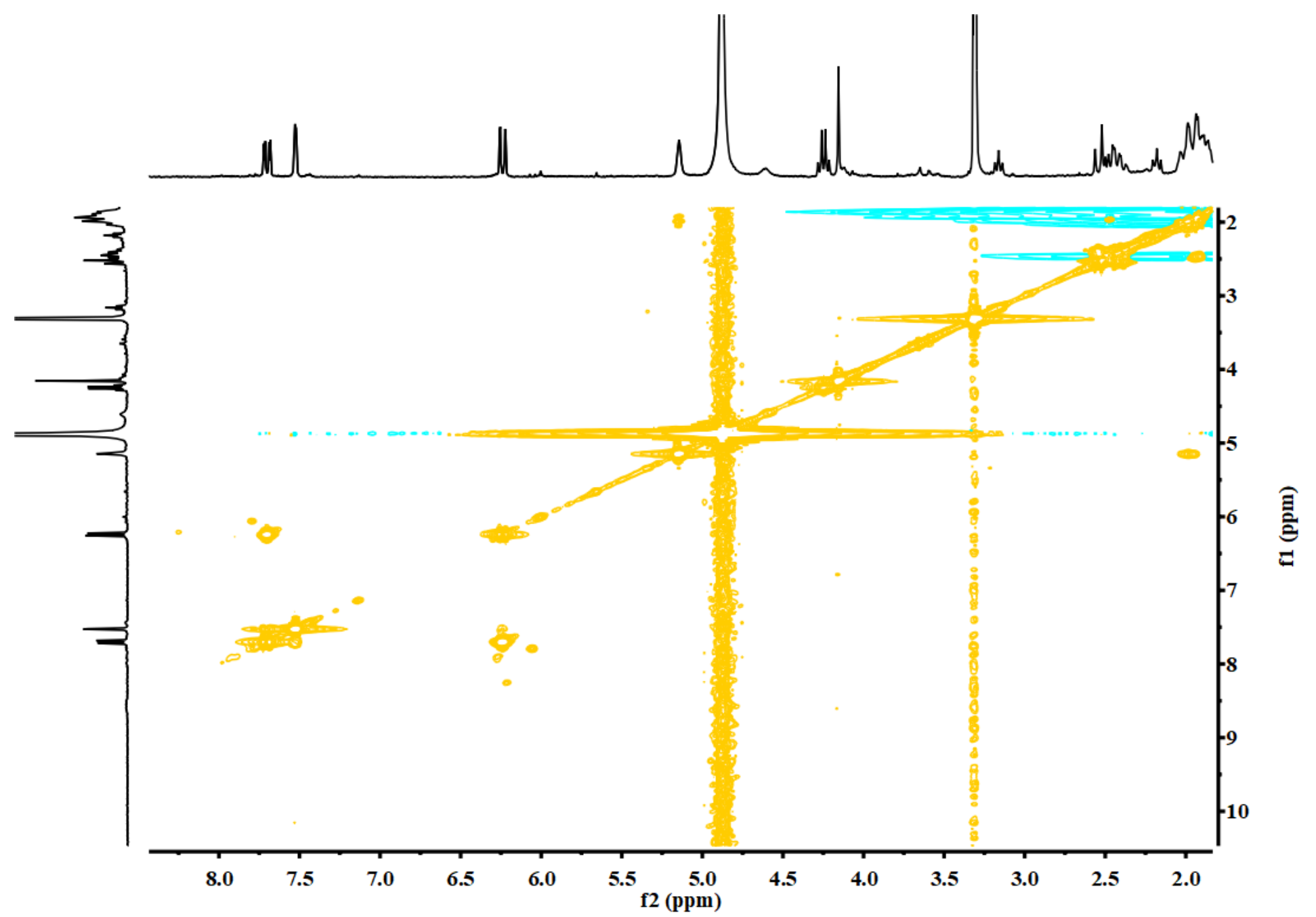

Figure S28. The ${ }^{1} \mathrm{H}^{-1} \mathrm{H}$ COSY spectrum of compound 3 (in $\mathrm{CD}_{3} \mathrm{OD}$ ). 


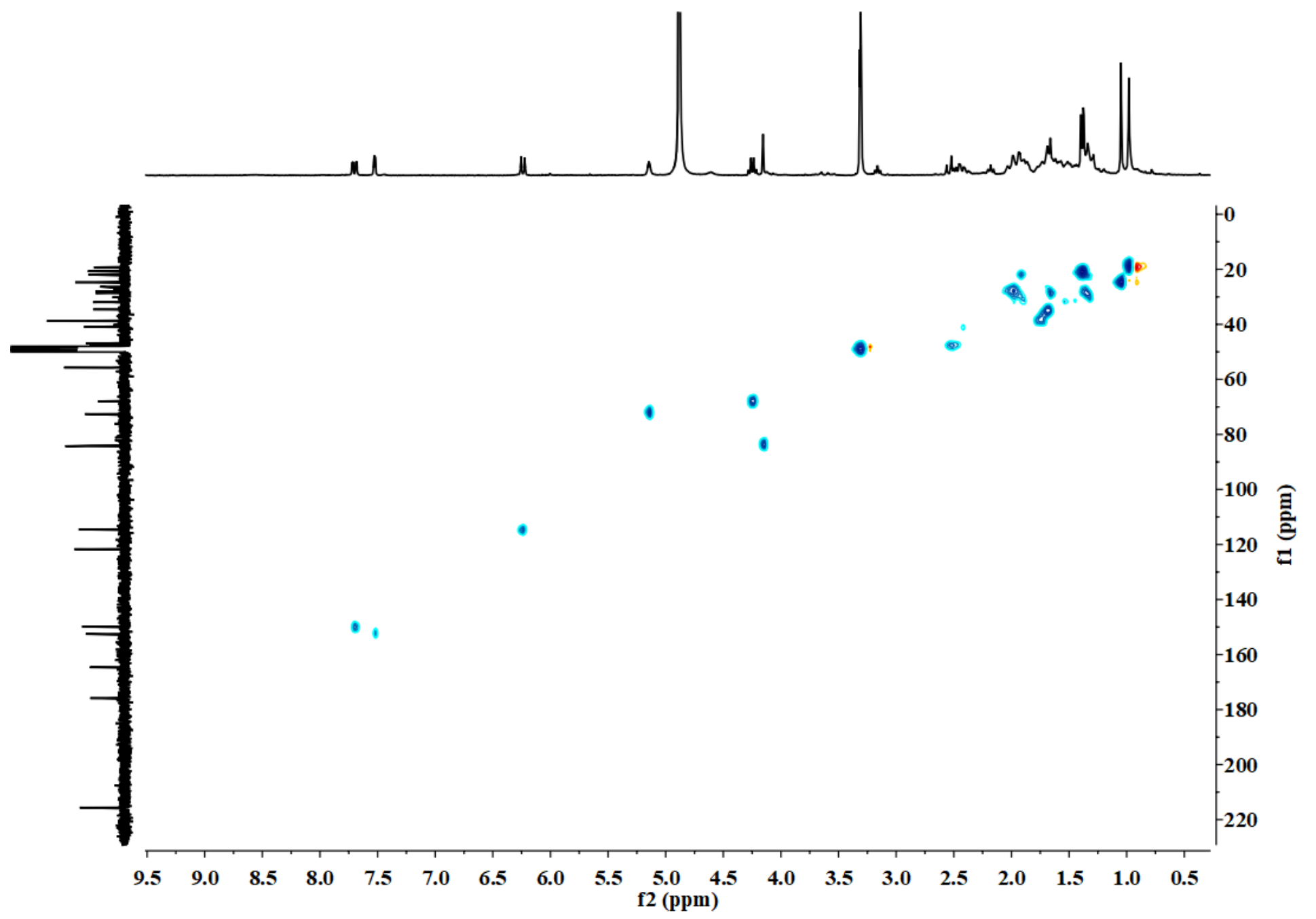

Figure S29. The HSQC spectrum of compound 3 (in $\mathrm{CD}_{3} \mathrm{OD}$ ). 


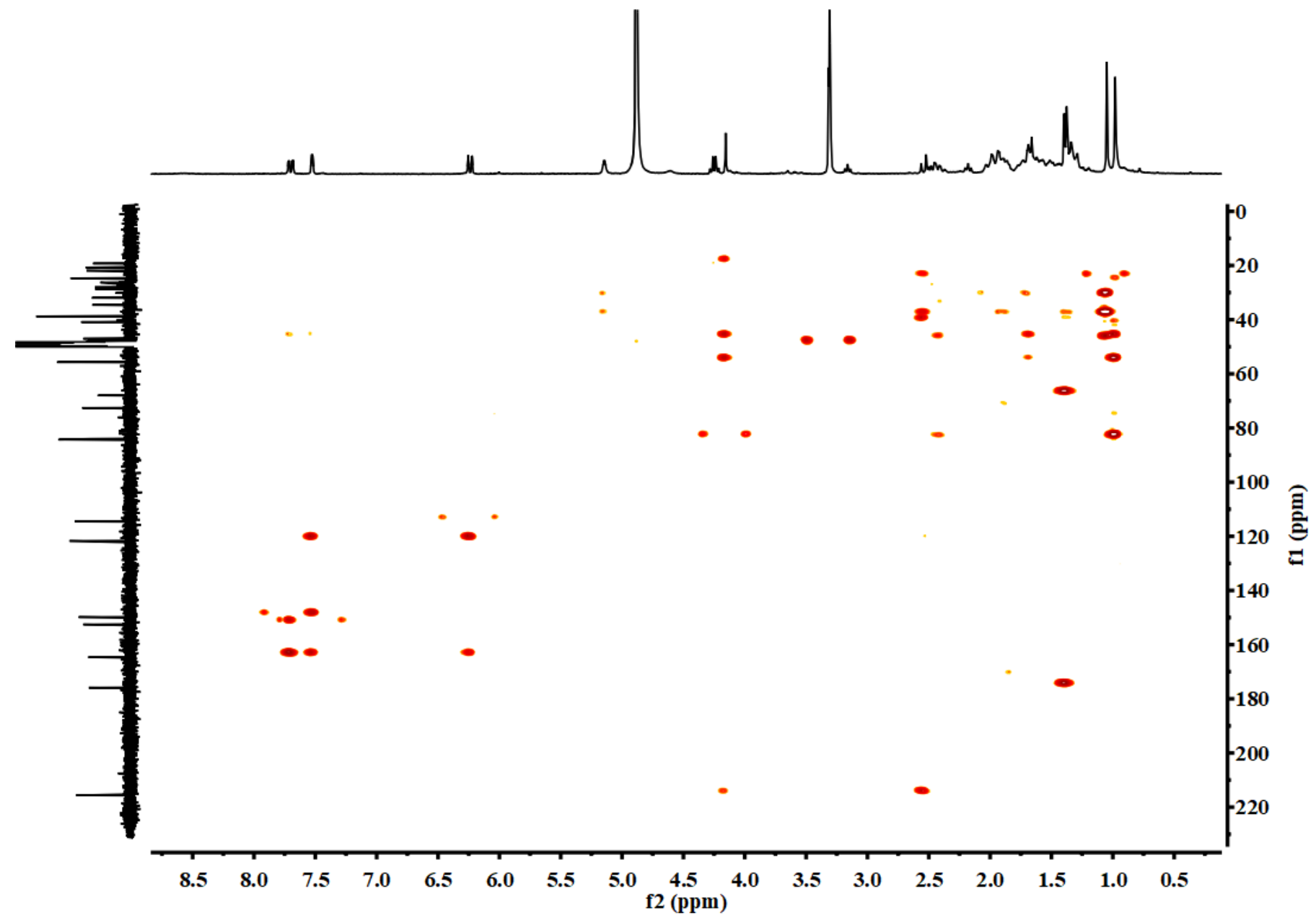

Figure S30. The HMBC spectrum of compound 3 (in $\mathrm{CD}_{3} \mathrm{OD}$ ). 


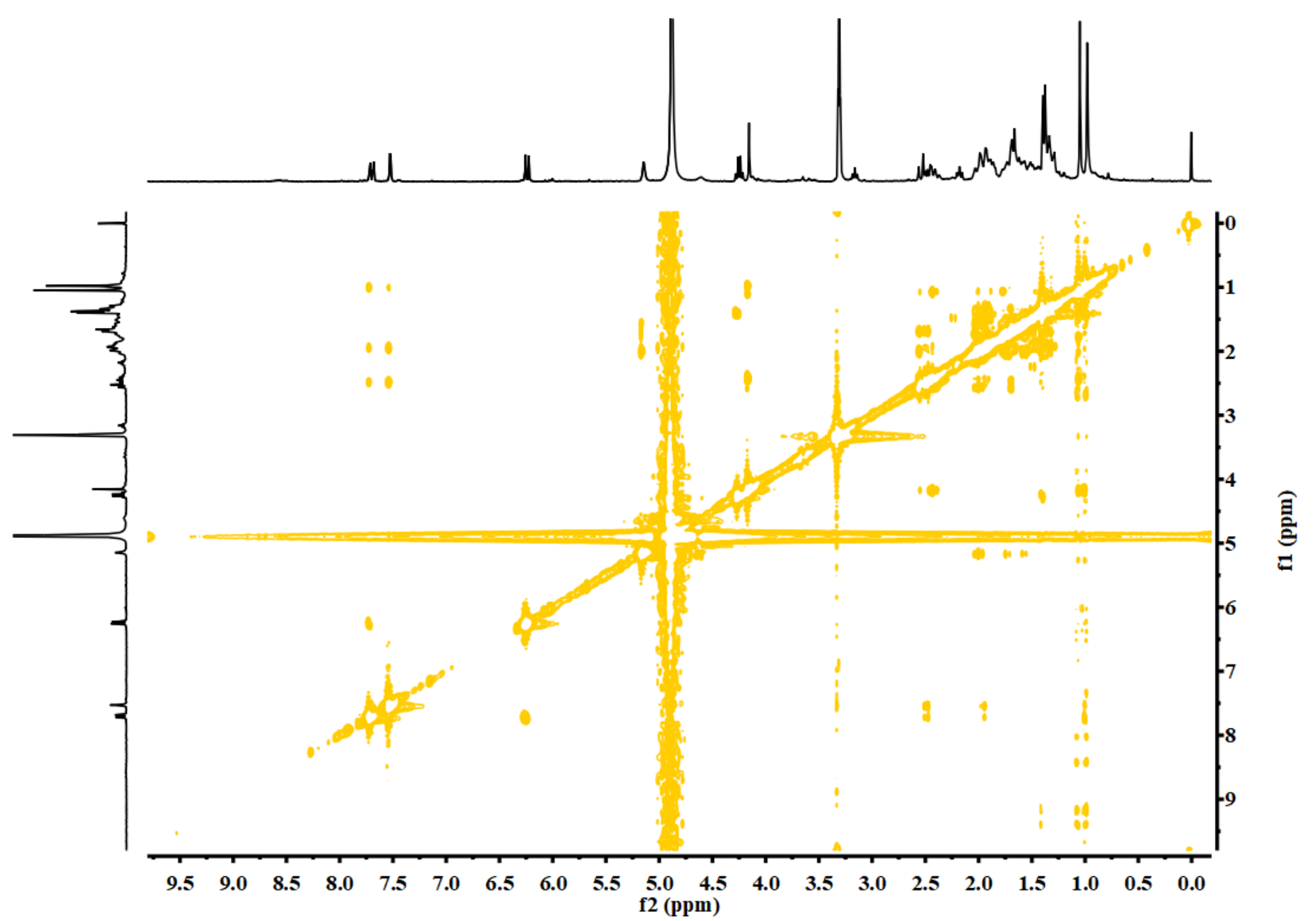

Figure S31. The NOESY spectrum of compound 3 (in $\mathrm{CD}_{3} \mathrm{OD}$ ). 


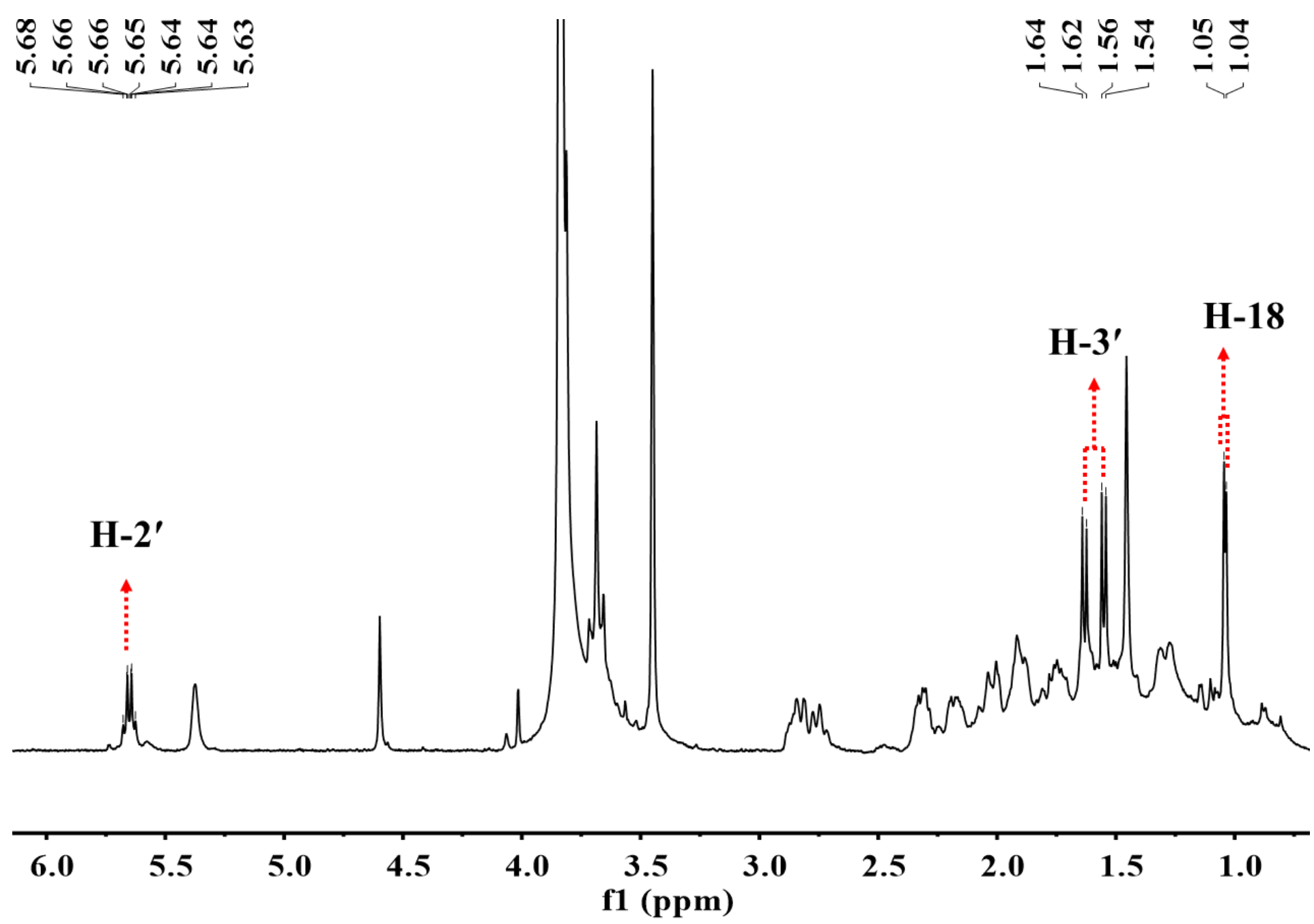

Figure S32. Enlarged ${ }^{1} \mathrm{H}$ NMR spectrum of (S)-MTPA-ester of compound 3 


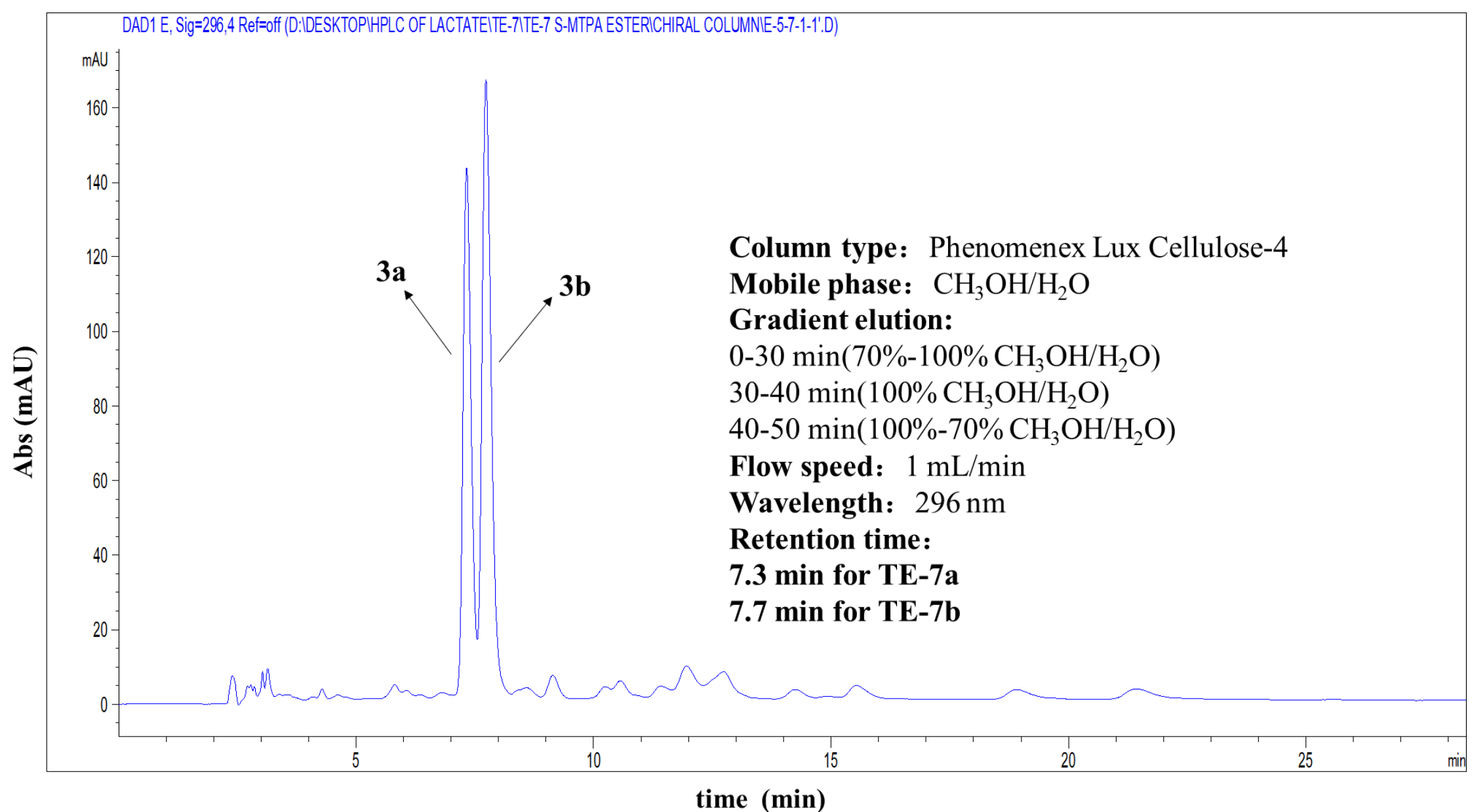

Figure S33. Chiral HPLC analysis result of compound 3 


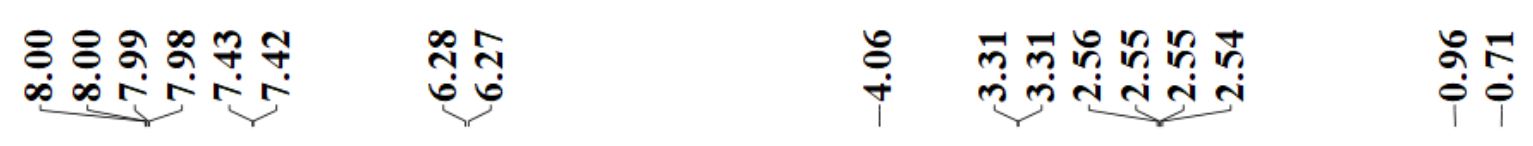

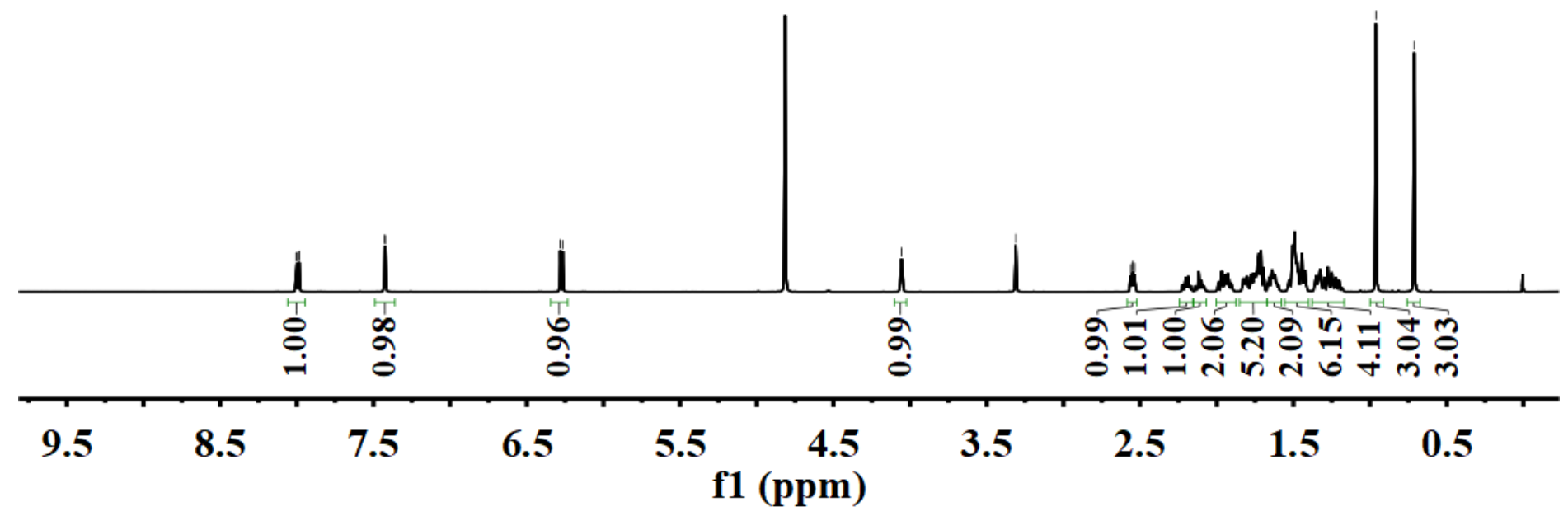

Figure S34. The ${ }^{1} \mathrm{H}-\mathrm{NMR}$ spectrum of bufalin (in $\mathrm{CD}_{3} \mathrm{OD}, 600 \mathrm{MHz}$ ). 


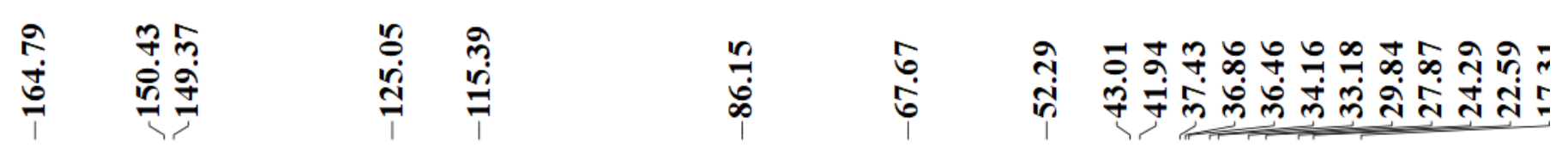

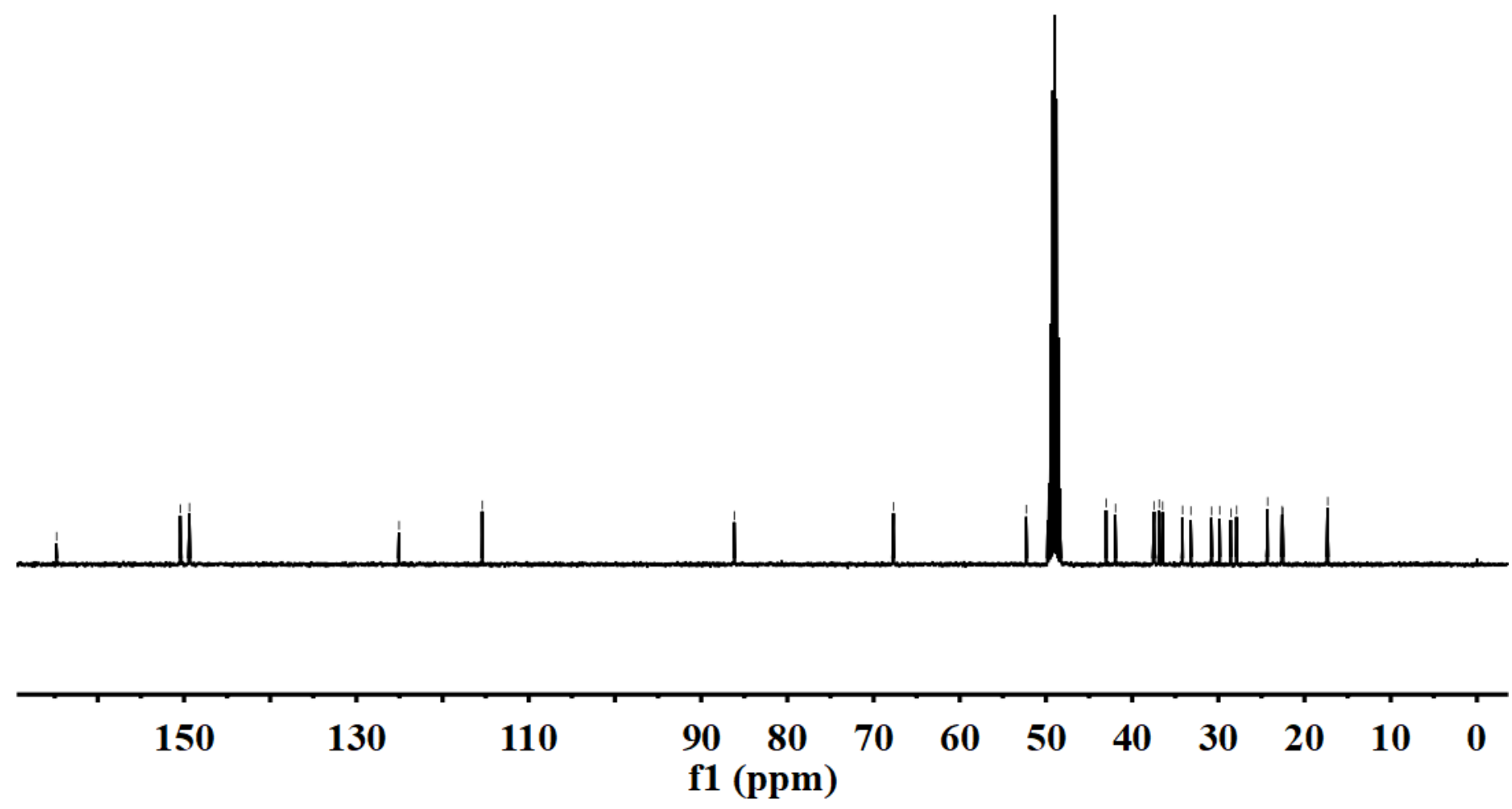

Figure S35. The ${ }^{13} \mathrm{C}-\mathrm{NMR}$ spectrum of bufalin (in $\mathrm{CD}_{3} \mathrm{OD}, 400 \mathrm{MHz}$ ). 

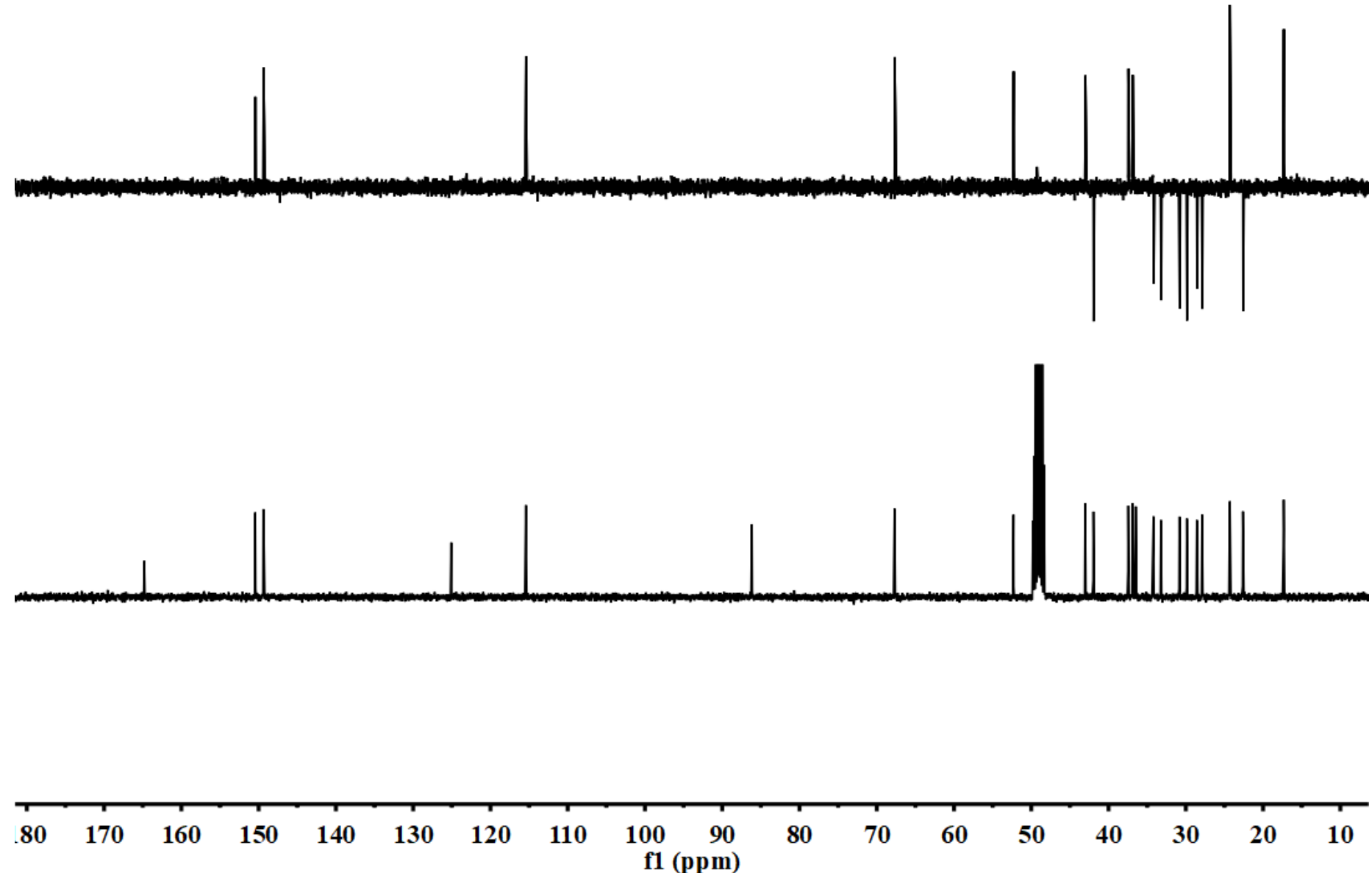

Figure S36. The ${ }^{13} \mathrm{C}-\mathrm{NMR}$ and DEPT 135 spectra of bufalin (in $\mathrm{CD}_{3} \mathrm{OD}$ ). 


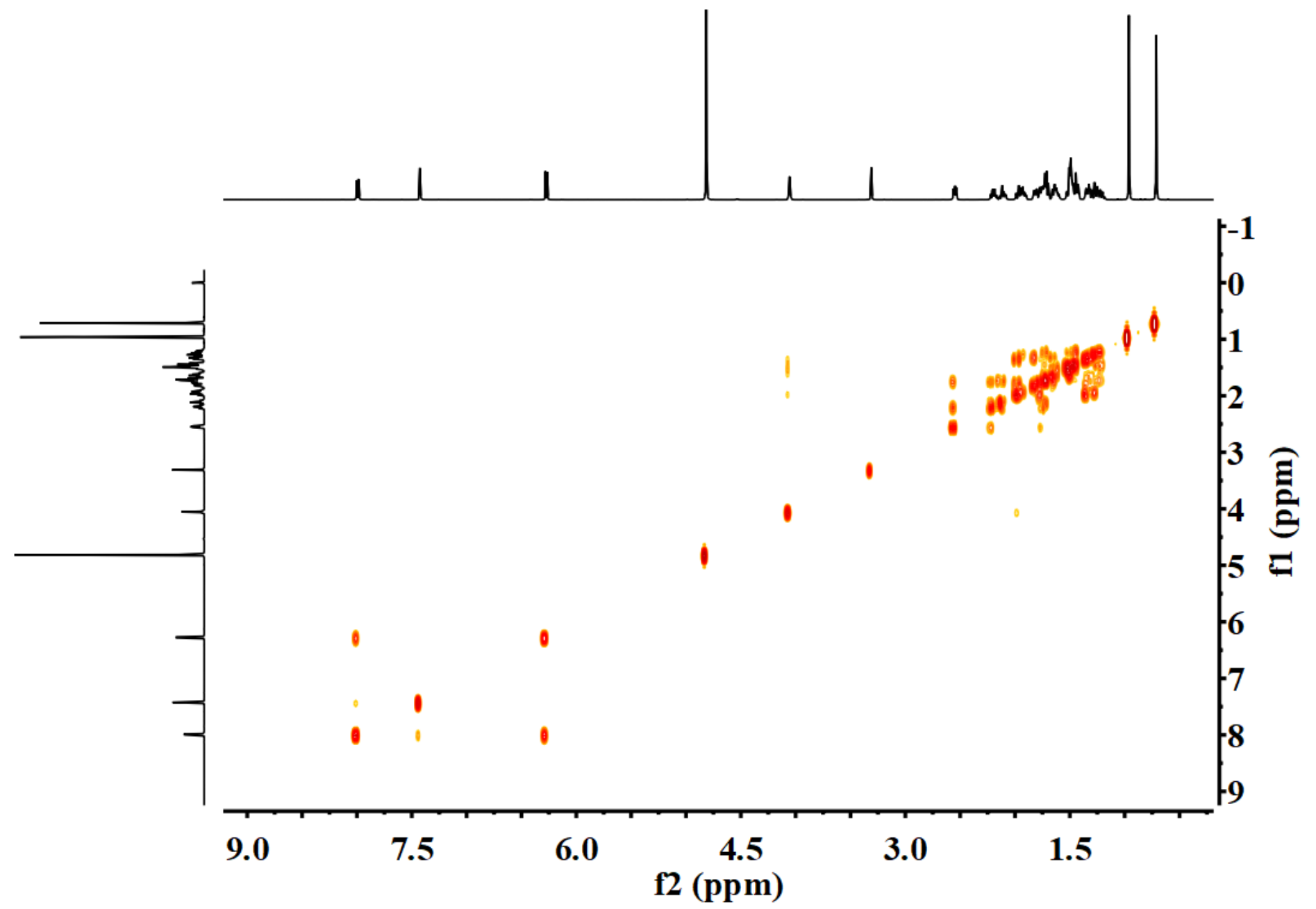

Figure S37. The ${ }^{1} \mathrm{H}^{-1} \mathrm{H}$ COSY spectrum of bufalin (in CD3OD). 


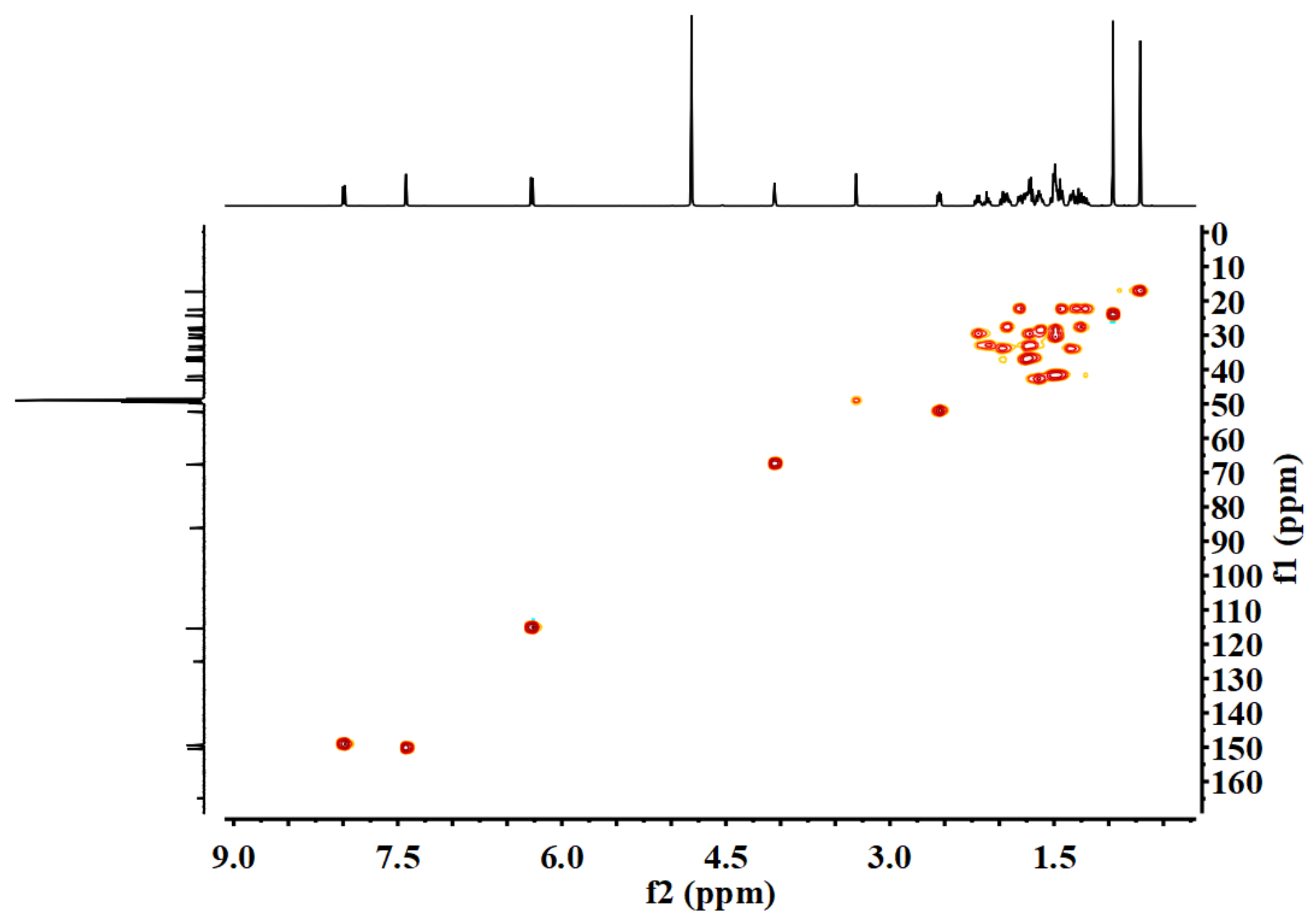

Figure S38.The HSQC spectrum of bufalin (in $\mathrm{CD}_{3} \mathrm{OD}$ ). 


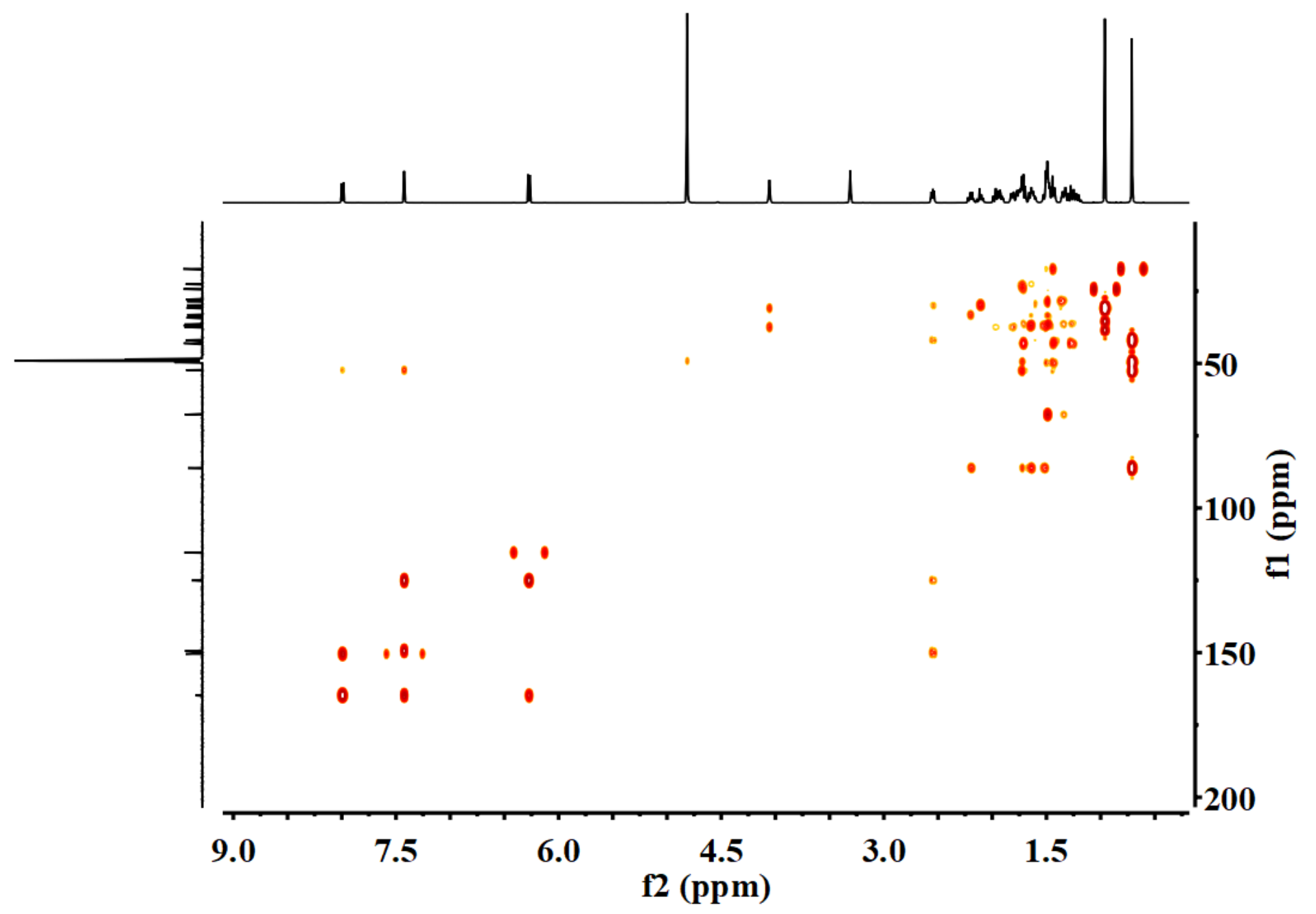

Figure S39. The $\mathrm{HMBC}$ spectrum of bufalin (in $\mathrm{CD}_{3} \mathrm{OD}$ ). 


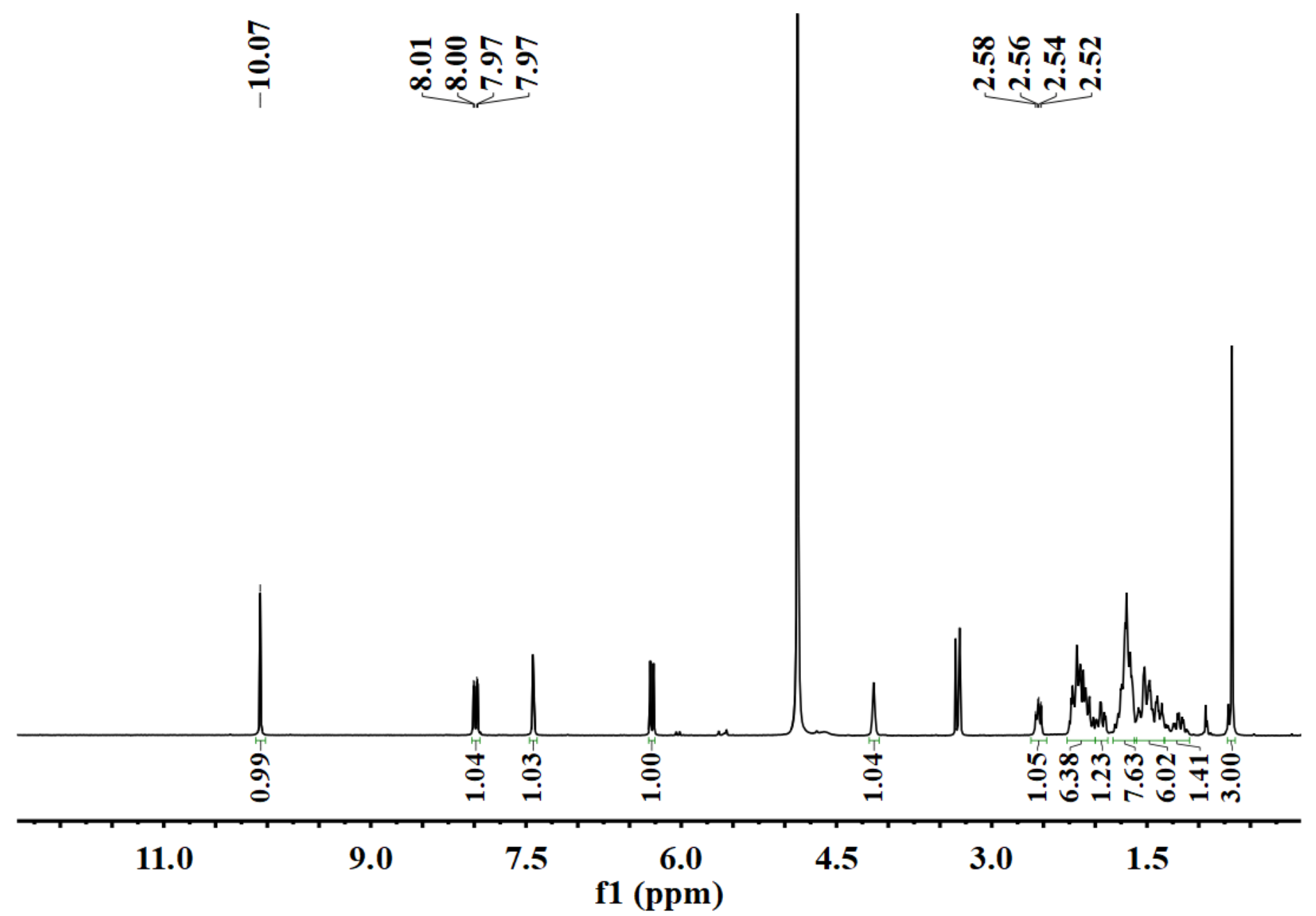

Figure S40. The ${ }^{1} \mathrm{H}-\mathrm{NMR}$ spectrum of hellebrigenin (6) (in $\mathrm{CD}_{3} \mathrm{OD}, 400 \mathrm{MHz}$ ) 


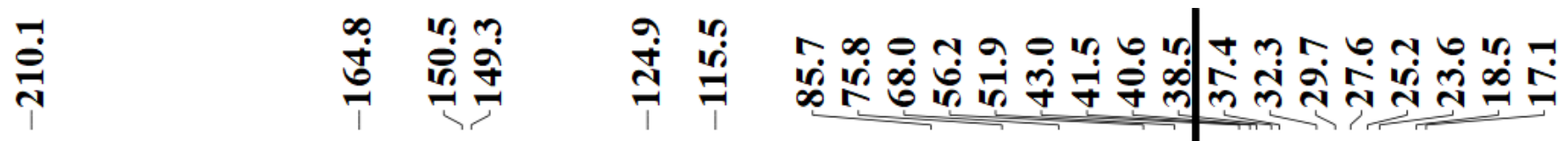

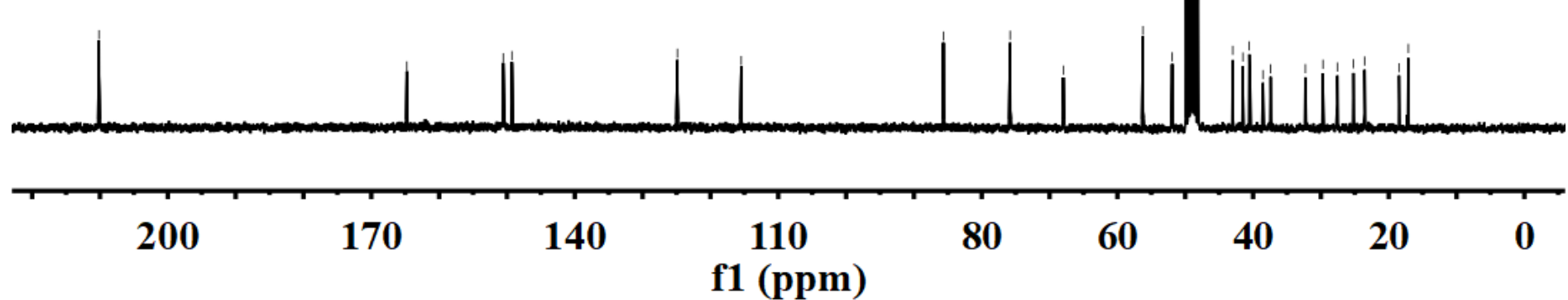

Figure S41. The ${ }^{13} \mathrm{C}-\mathrm{NMR}$ spectrum of hellebrigenin (6) (in $\mathrm{CD}_{3} \mathrm{OD}, 400 \mathrm{MHz}$ ) 


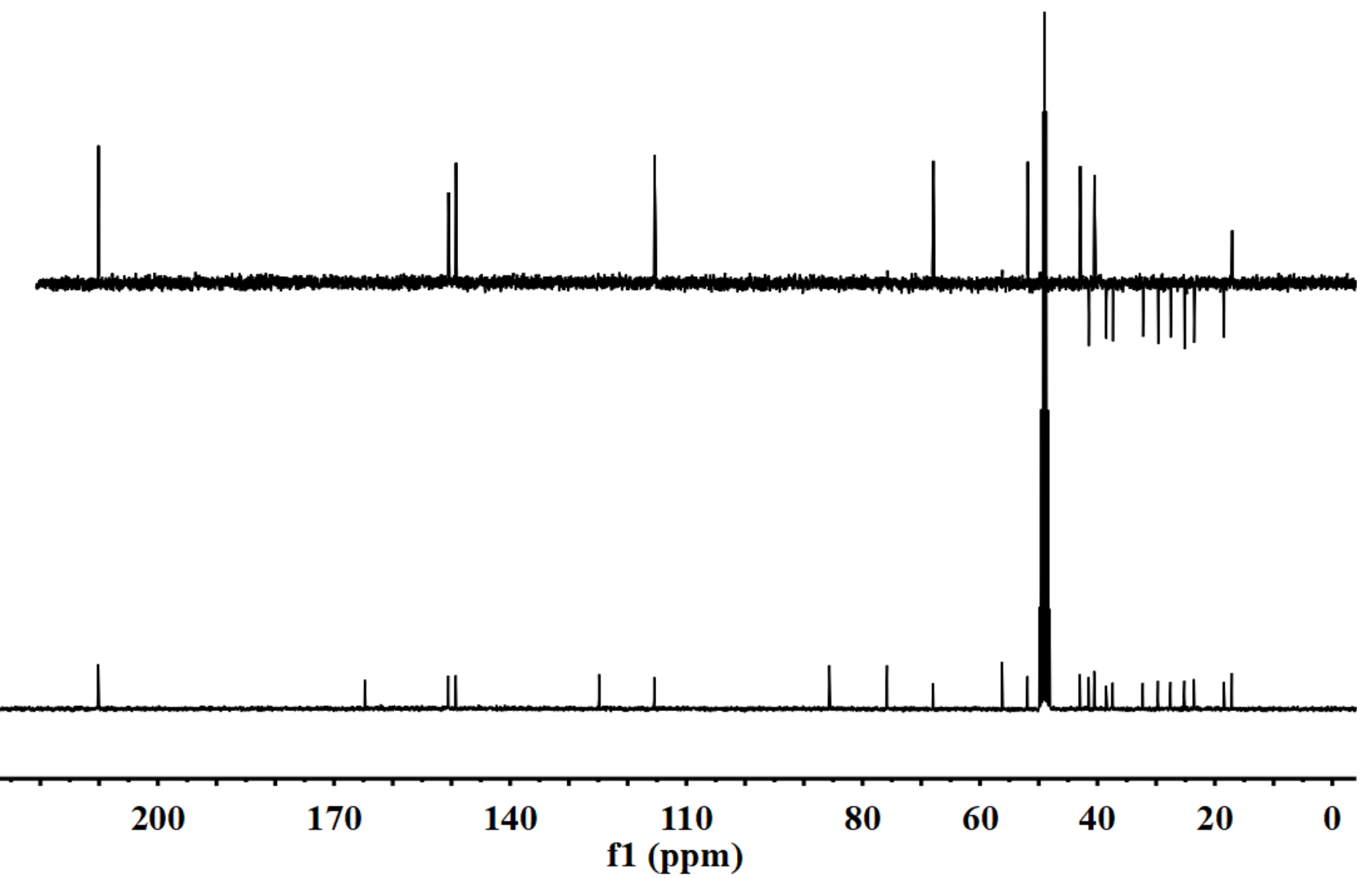

Figure S42. The ${ }^{13} \mathrm{C}-\mathrm{NMR}$ and DEPT 135 spectra of hellebrigenin (6) (in $\mathrm{CD}_{3} \mathrm{OD}$ ). 


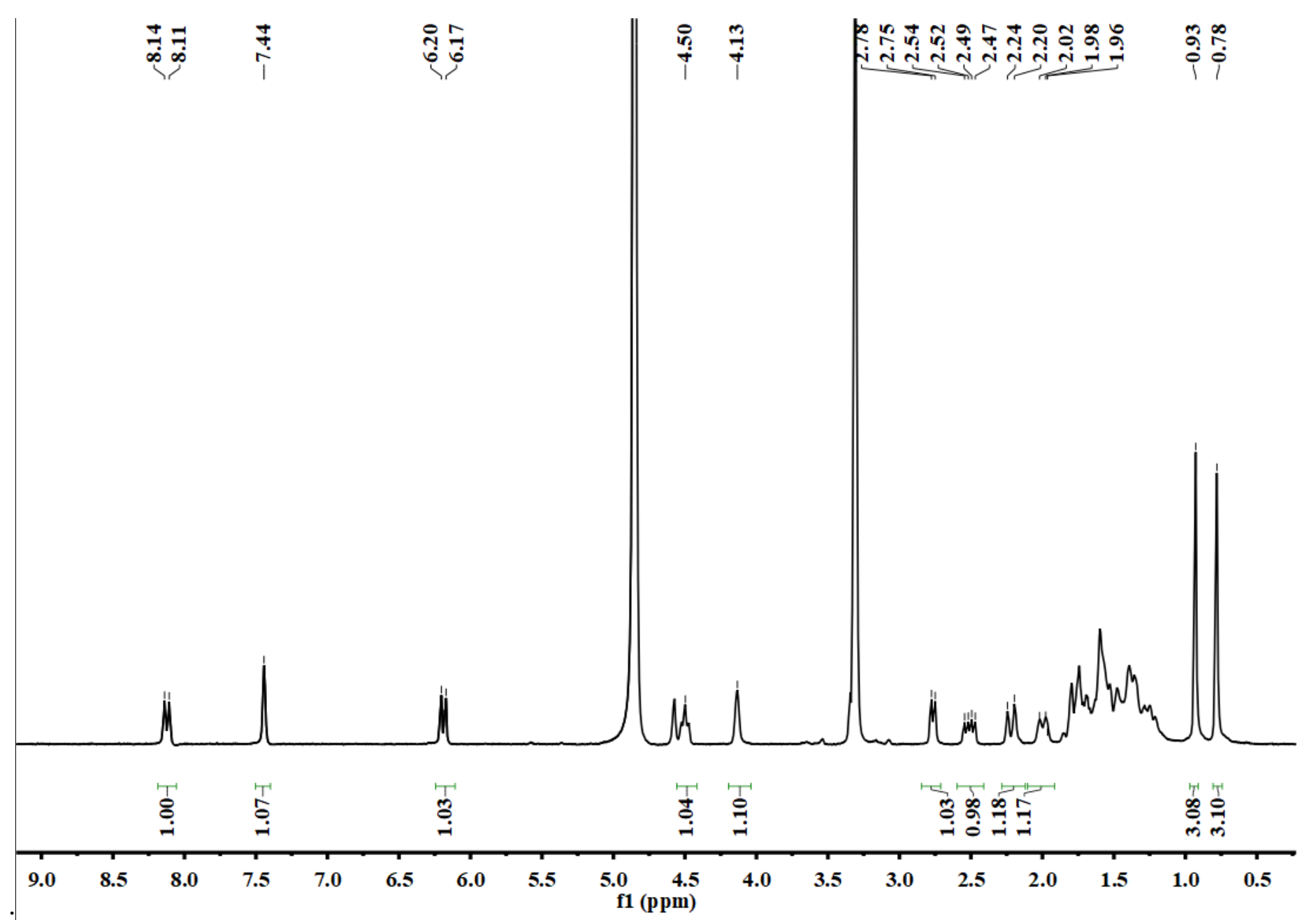

Figure S43. The ${ }^{1} \mathrm{H}-\mathrm{NMR}$ spectrum of 4 (in $\mathrm{CD}_{3} \mathrm{OD}, 400 \mathrm{MHz}$ ) 


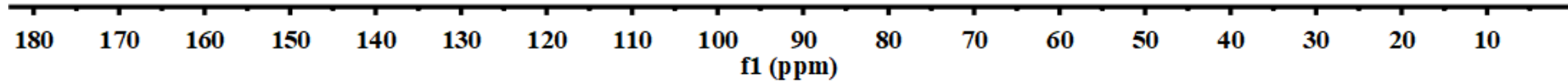

Figure $\mathbf{S 4 4}$. The ${ }^{13} \mathrm{C}-\mathrm{NMR}$ spectrum of $\mathbf{4}$ (in $\mathrm{CD}_{3} \mathrm{OD}, 400 \mathrm{MHz}$ ) 


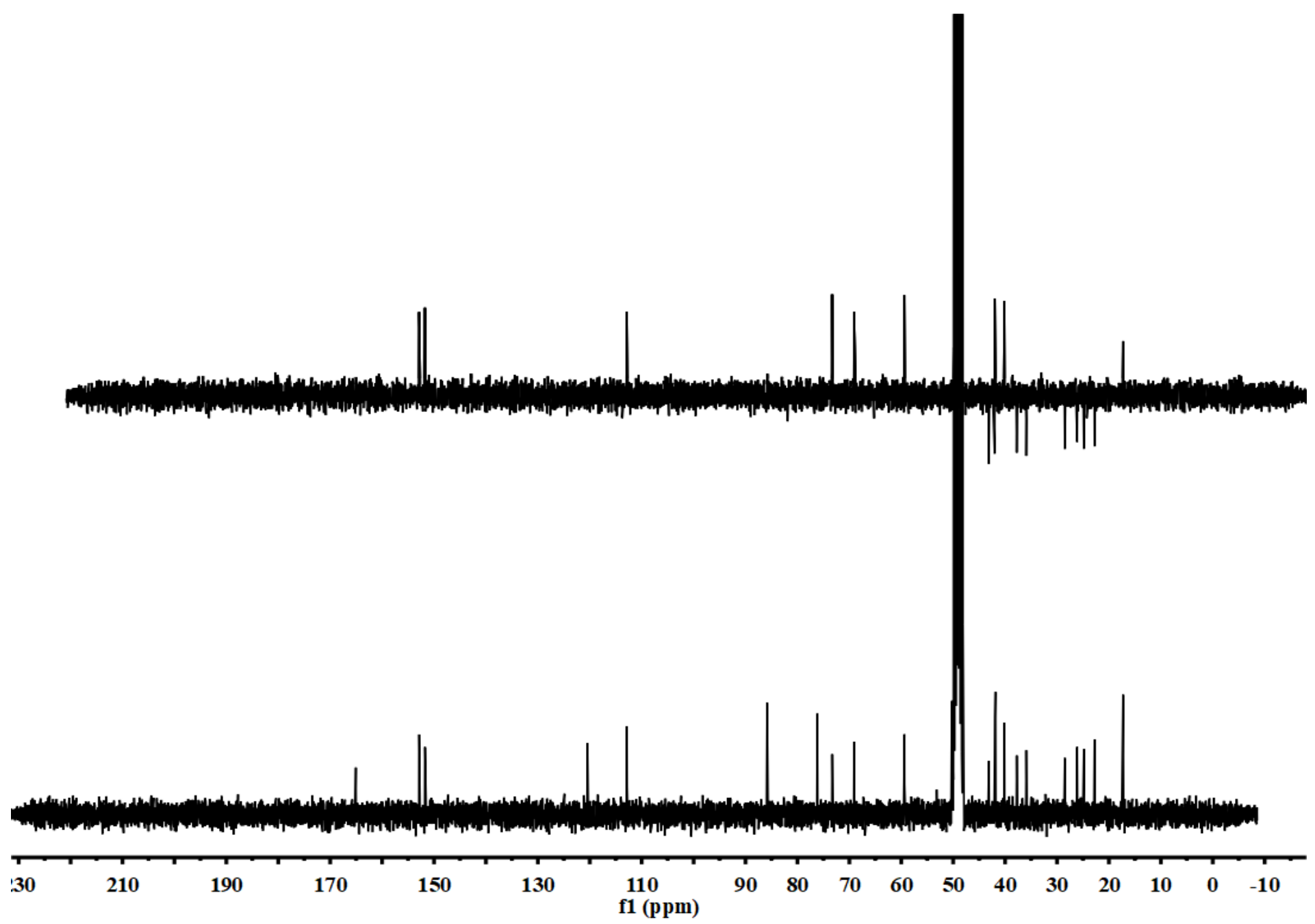

Figure S45. The ${ }^{13} \mathrm{C}-\mathrm{NMR}$ and DEPT 135 spectra of $\mathbf{4}$ (in $\mathrm{CD}_{3} \mathrm{OD}, 400 \mathrm{MHz}$ ) 


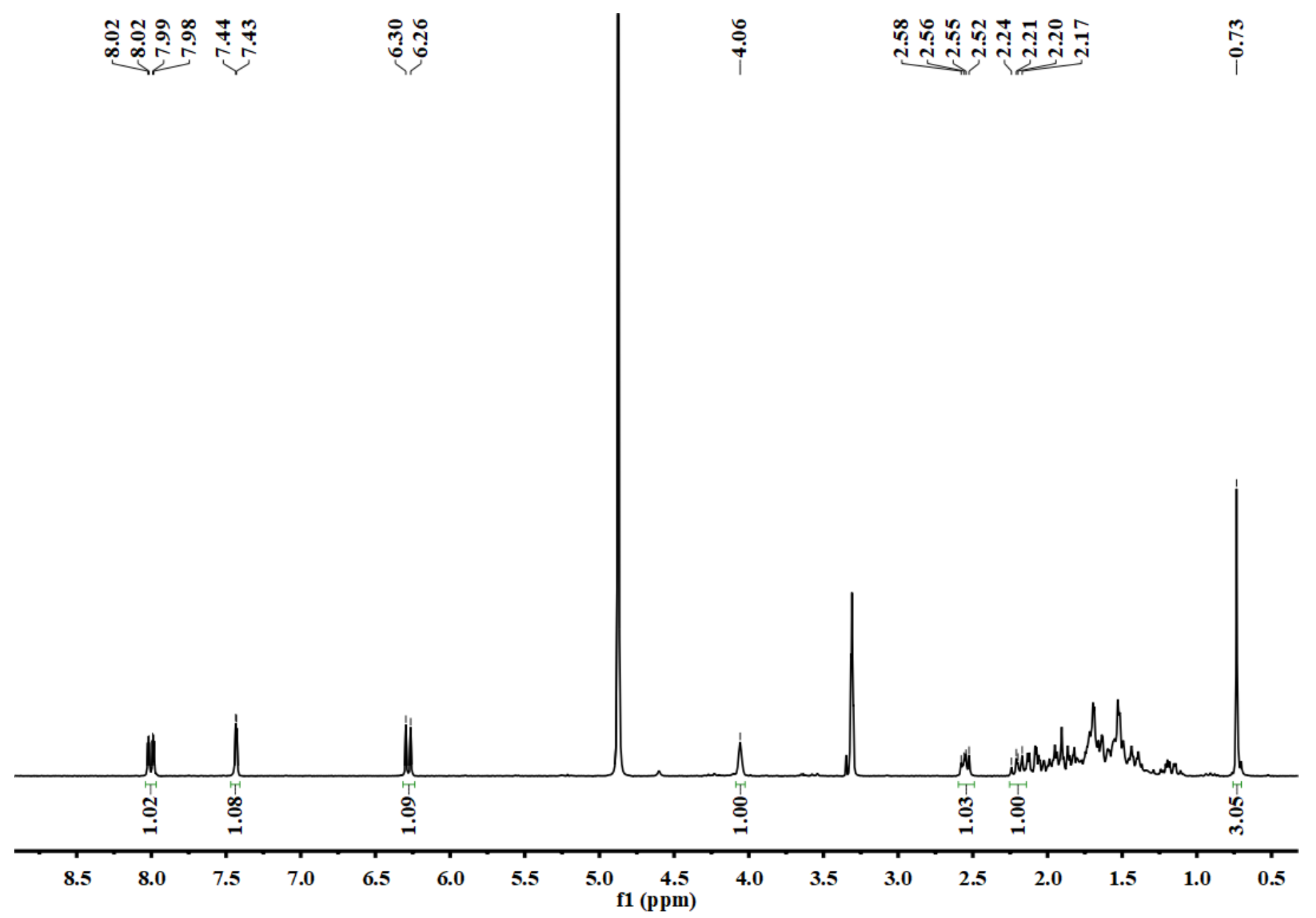

Figure S46. The ${ }^{1} \mathrm{H}-\mathrm{NMR}$ spectrum of 5 (in $\mathrm{CD}_{3} \mathrm{OD}, 400 \mathrm{MHz}$ ) 


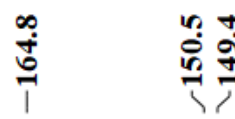

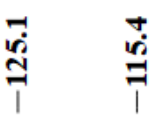

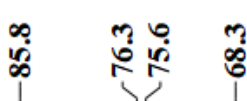

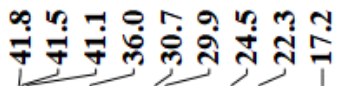
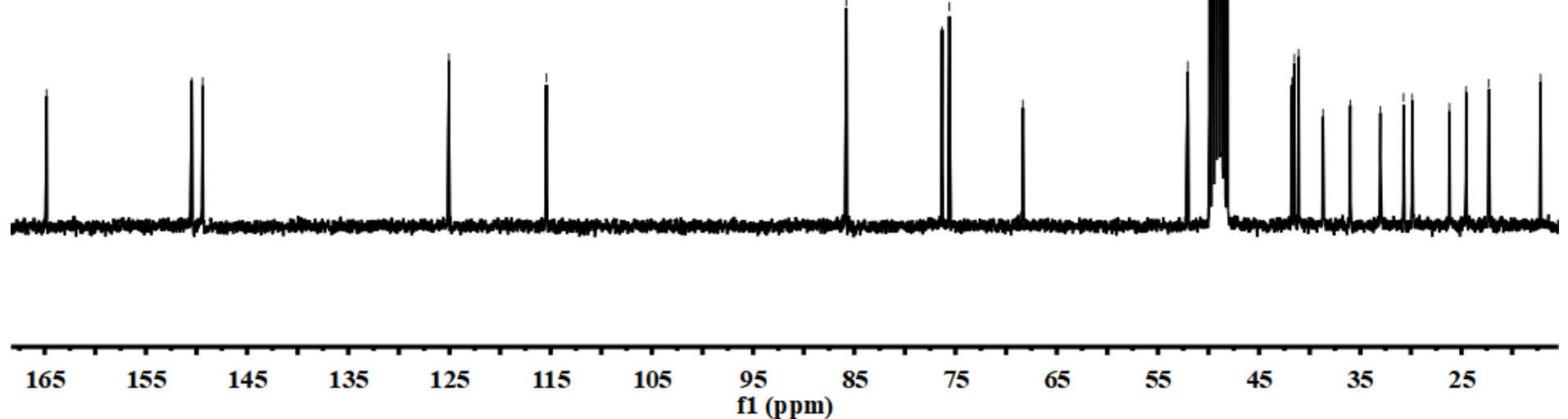

Figure S47. The ${ }^{13} \mathrm{C}-\mathrm{NMR}$ spectrum of 5 (in $\mathrm{CD}_{3} \mathrm{OD}, 400 \mathrm{MHz}$ ) 


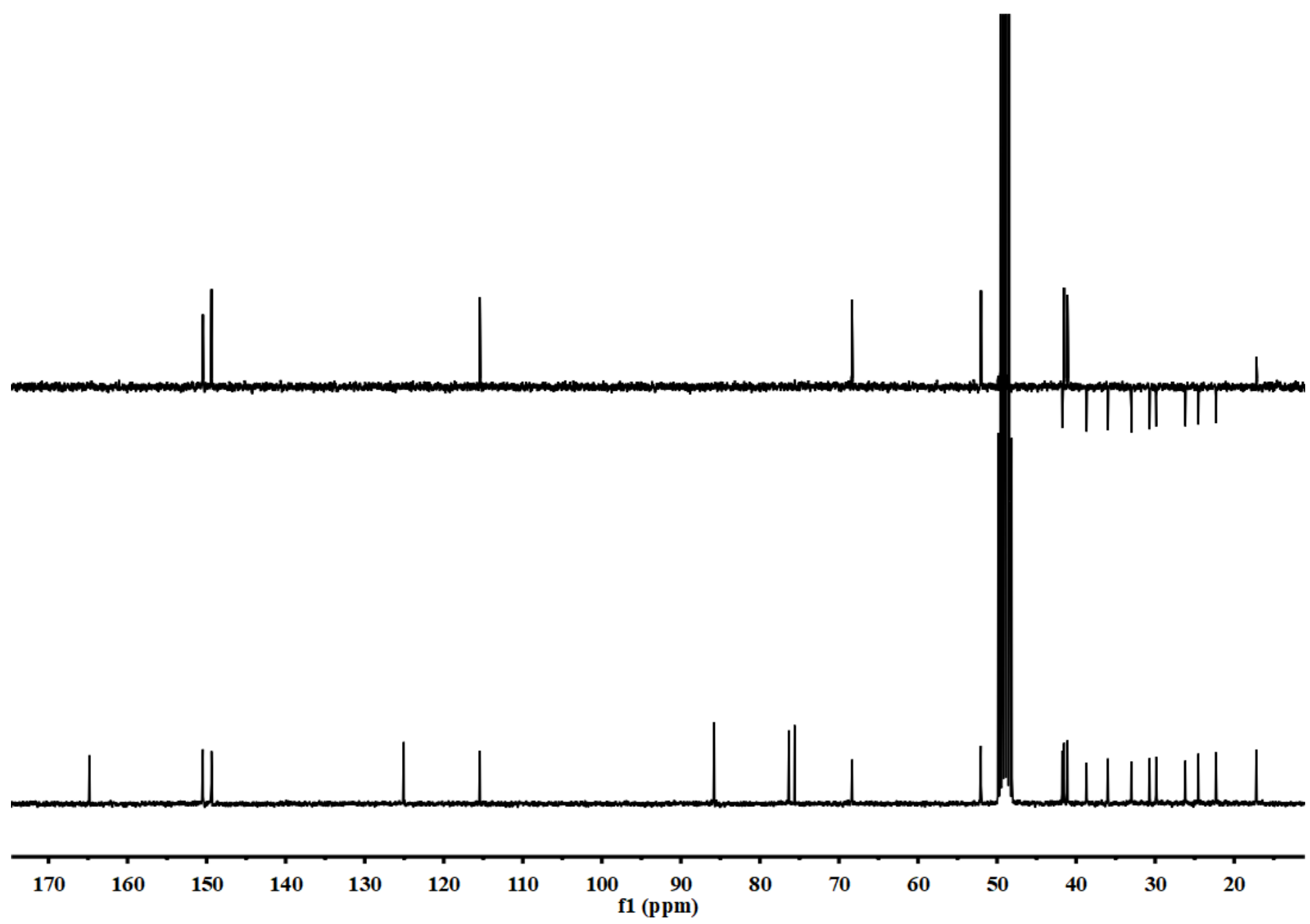

Figure S48. The ${ }^{13} \mathrm{C}-\mathrm{NMR}$ and DEPT 135 spectra of 5 (in $\mathrm{CD}_{3} \mathrm{OD}, 400 \mathrm{MHz}$ ) 


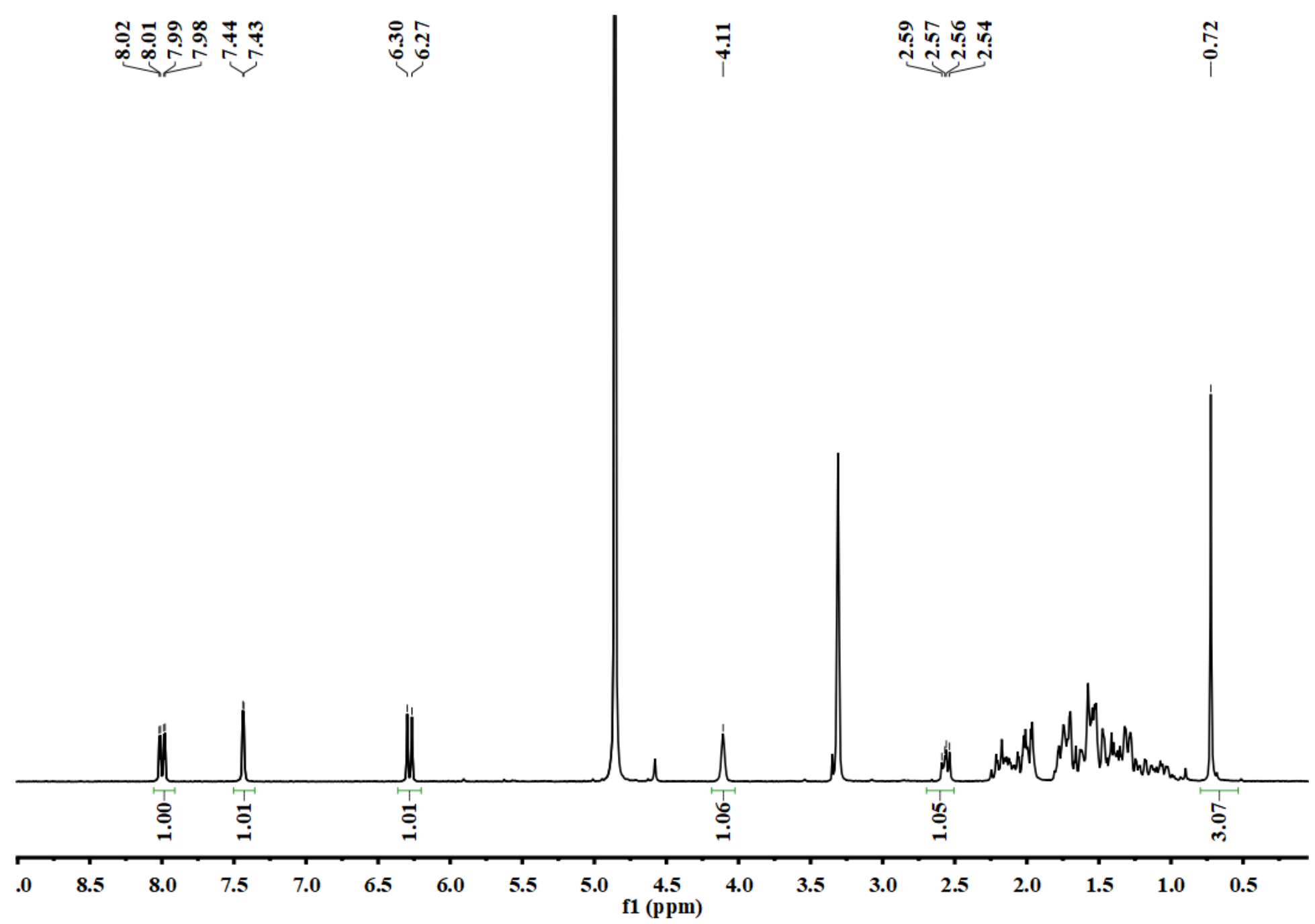

Figure S49. The ${ }^{1} \mathrm{H}-\mathrm{NMR}$ spectrum of 7 (in $\mathrm{CD}_{3} \mathrm{OD}, 400 \mathrm{MHz}$ ) 


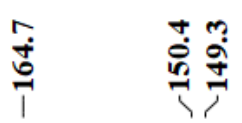

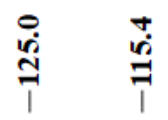
事蓄

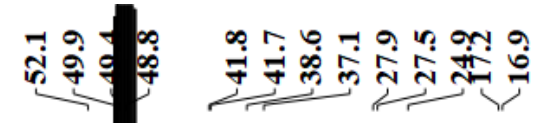
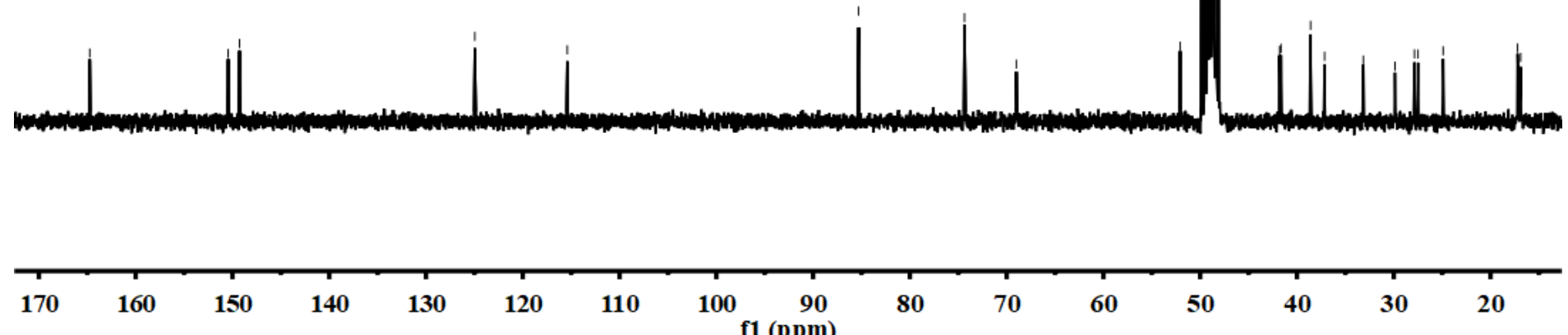

Figure S50. The ${ }^{13} \mathrm{C}-\mathrm{NMR}$ spectrum of 7 (in $\mathrm{CD}_{3} \mathrm{OD}, 400 \mathrm{MHz}$ ) 


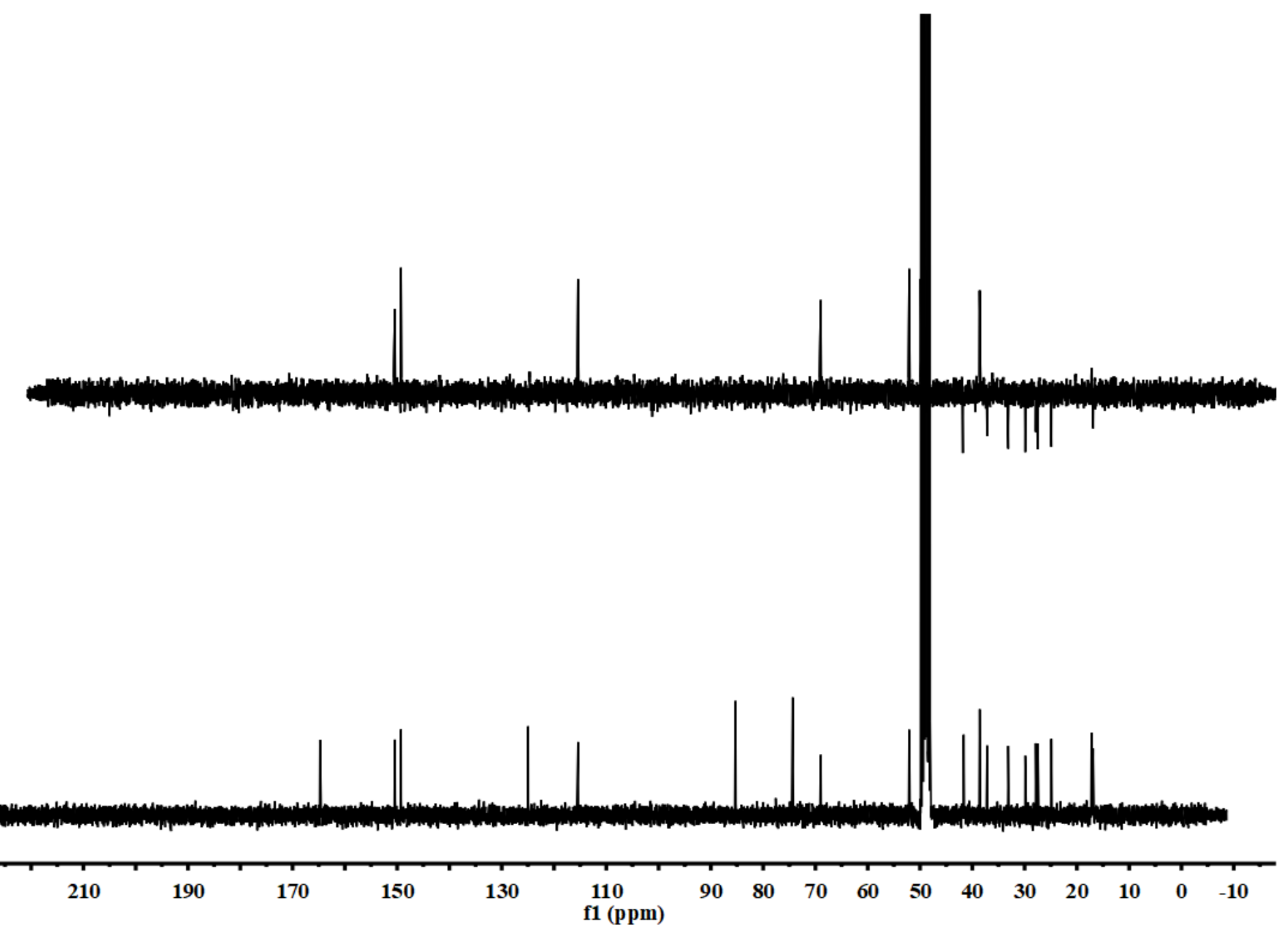

Figure S51. The ${ }^{13} \mathrm{C}-\mathrm{NMR}$ and DEPT 135 spectra of 7 (in $\mathrm{CD}_{3} \mathrm{OD}, 400 \mathrm{MHz}$ ) 


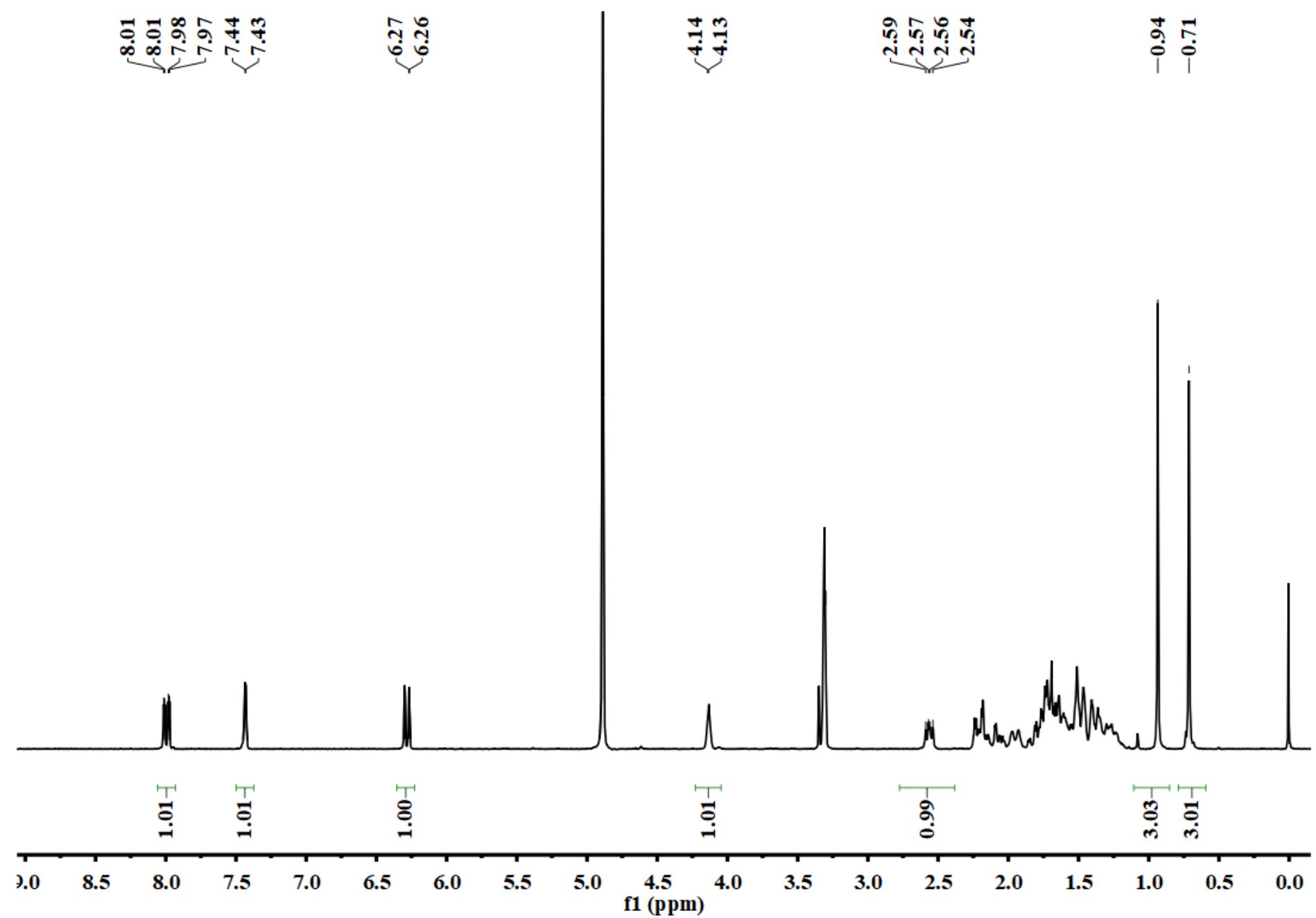

Figure S52. The ${ }^{1} \mathrm{H}-\mathrm{NMR}$ spectrum of 8 (in $\mathrm{CD}_{3} \mathrm{OD}, 400 \mathrm{MHz}$ ) 


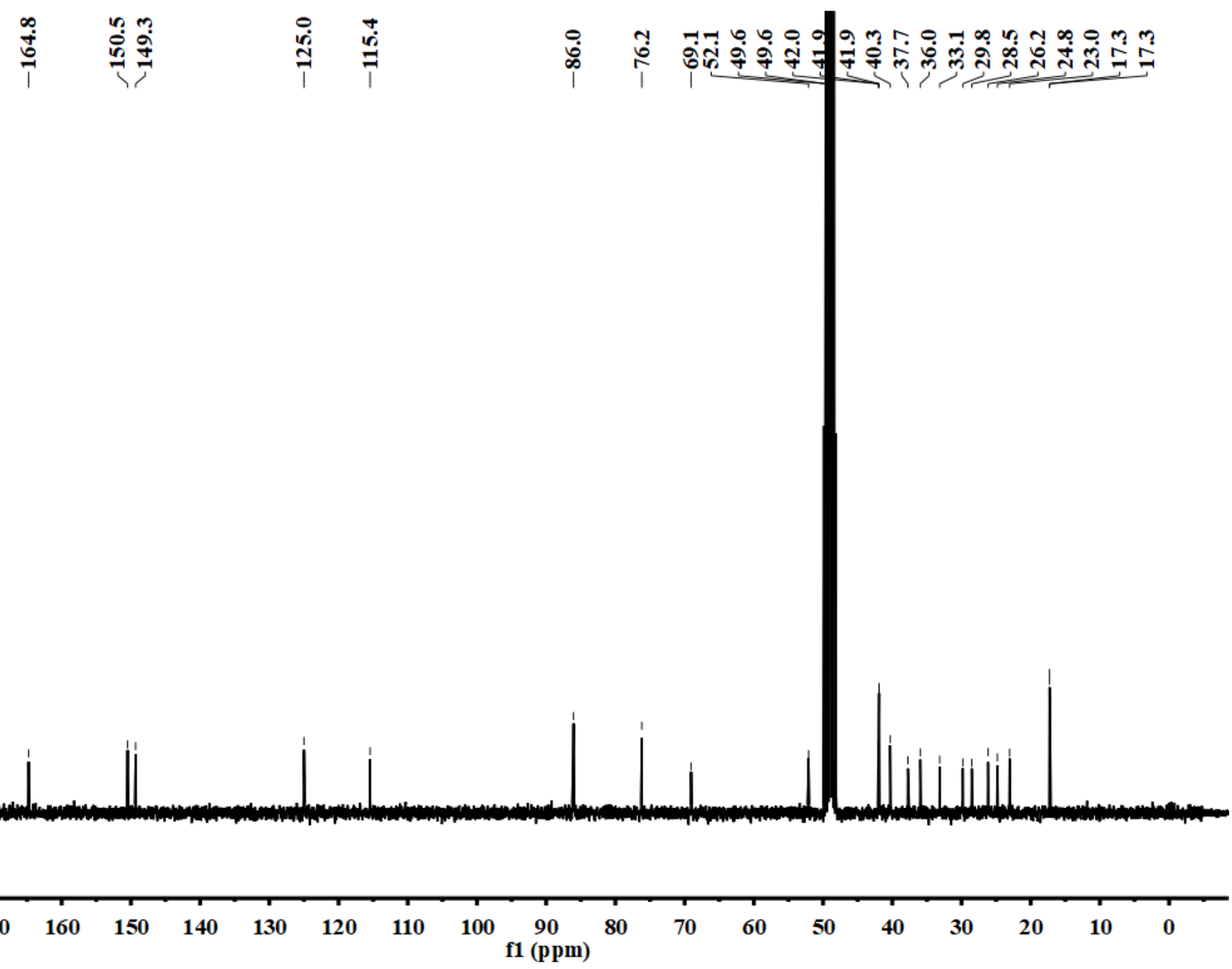

Figure S53. The ${ }^{13} \mathrm{C}-\mathrm{NMR}$ spectrum of $\mathbf{8}$ (in $\mathrm{CD}_{3} \mathrm{OD}, 400 \mathrm{MHz}$ ) 


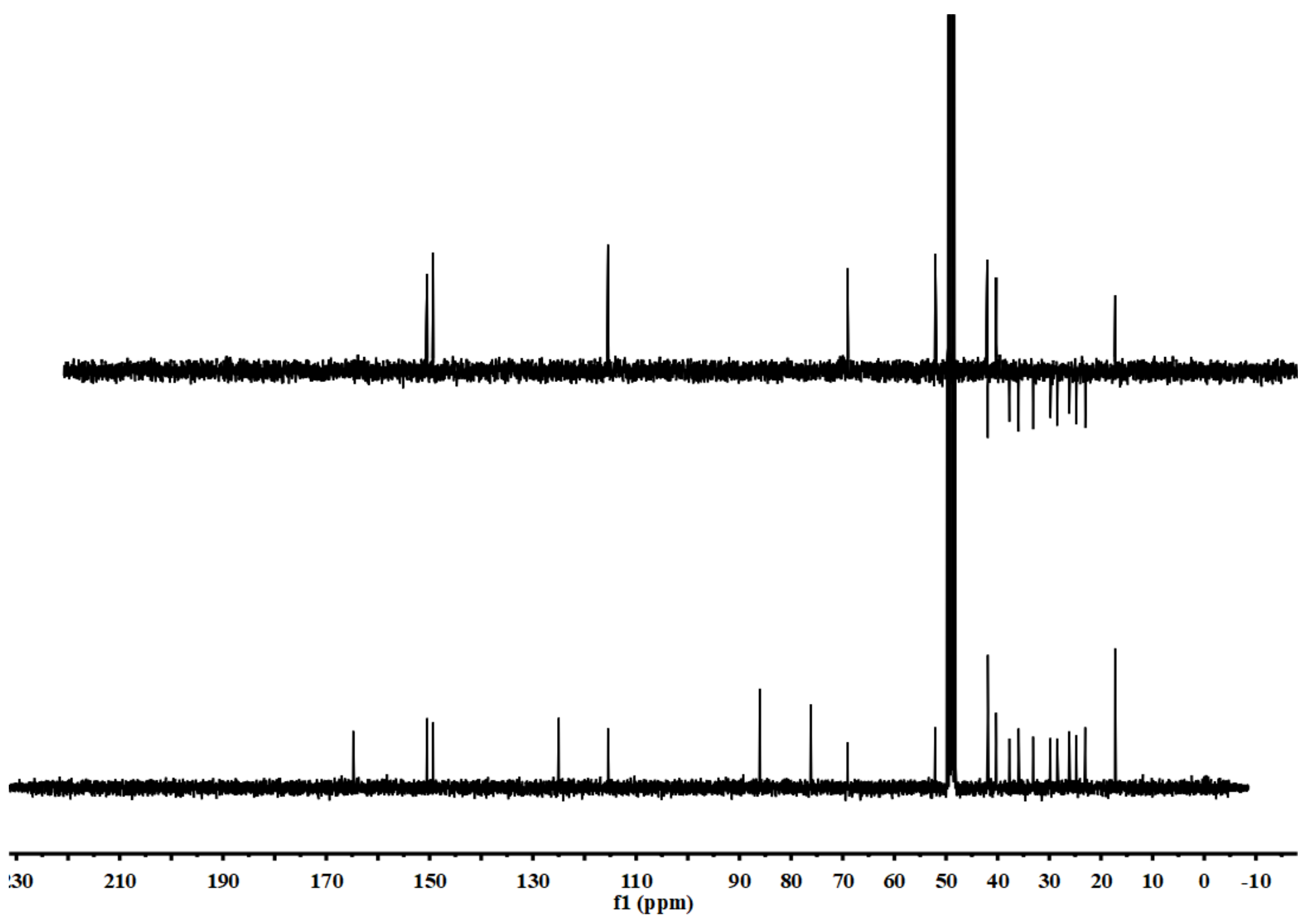

Figure S54. The ${ }^{13} \mathrm{C}-\mathrm{NMR}$ and DEPT 135 spectra of 8 (in $\mathrm{CD}_{3} \mathrm{OD}, 400 \mathrm{MHz}$ ) 


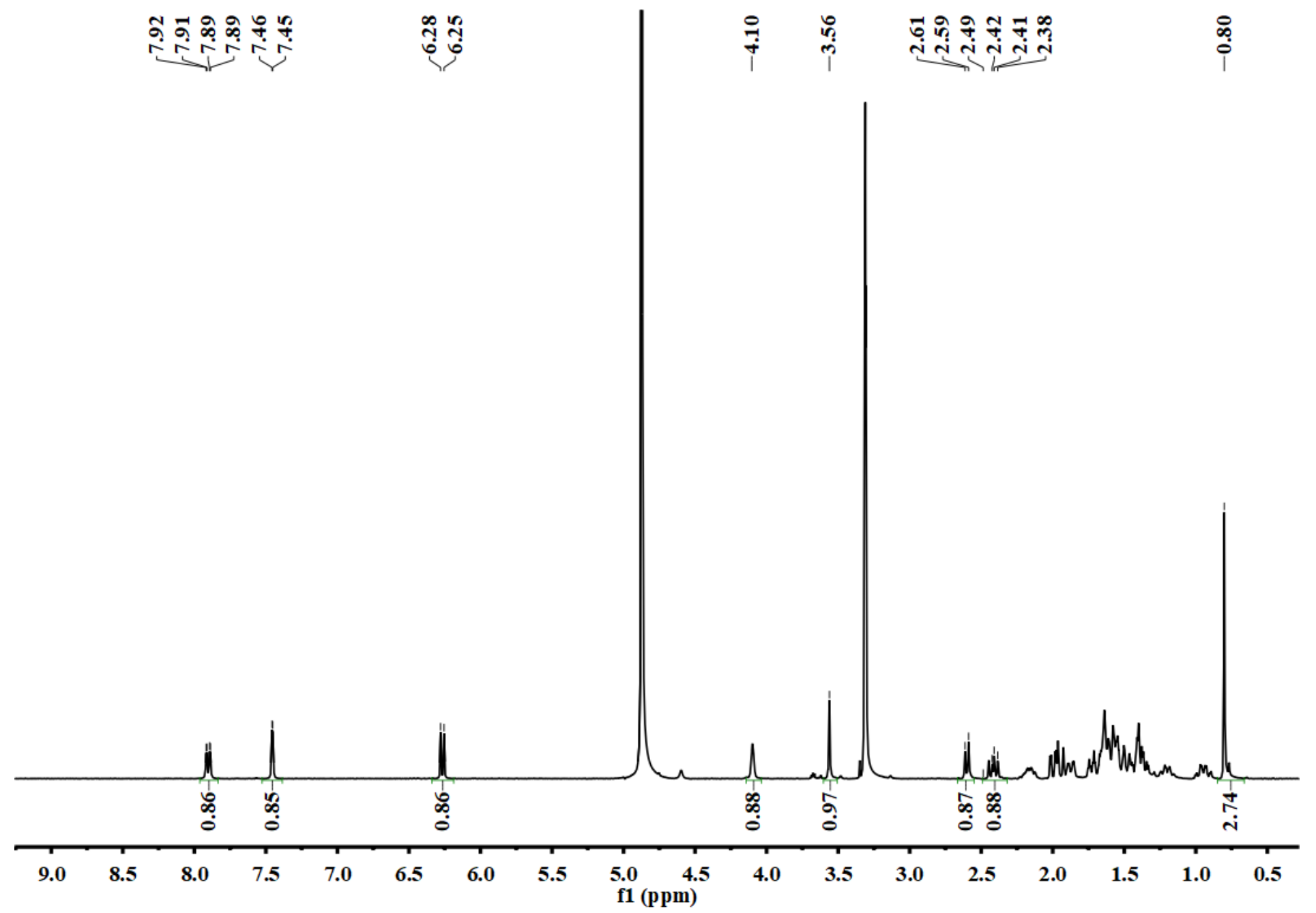

Figure S55. The ${ }^{1} \mathrm{H}-\mathrm{NMR}$ spectrum of 9 (in $\mathrm{CD}_{3} \mathrm{OD}, 400 \mathrm{MHz}$ ) 


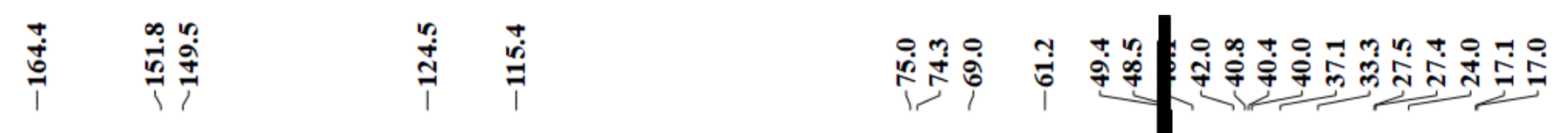
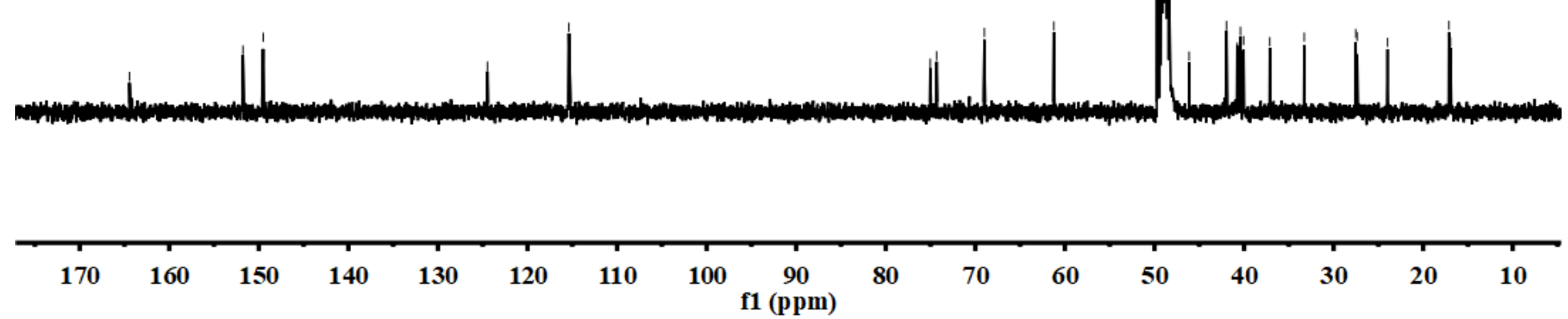

Figure S56. The ${ }^{13} \mathrm{C}-\mathrm{NMR}$ spectrum of 9 (in $\mathrm{CD}_{3} \mathrm{OD}, 400 \mathrm{MHz}$ ) 


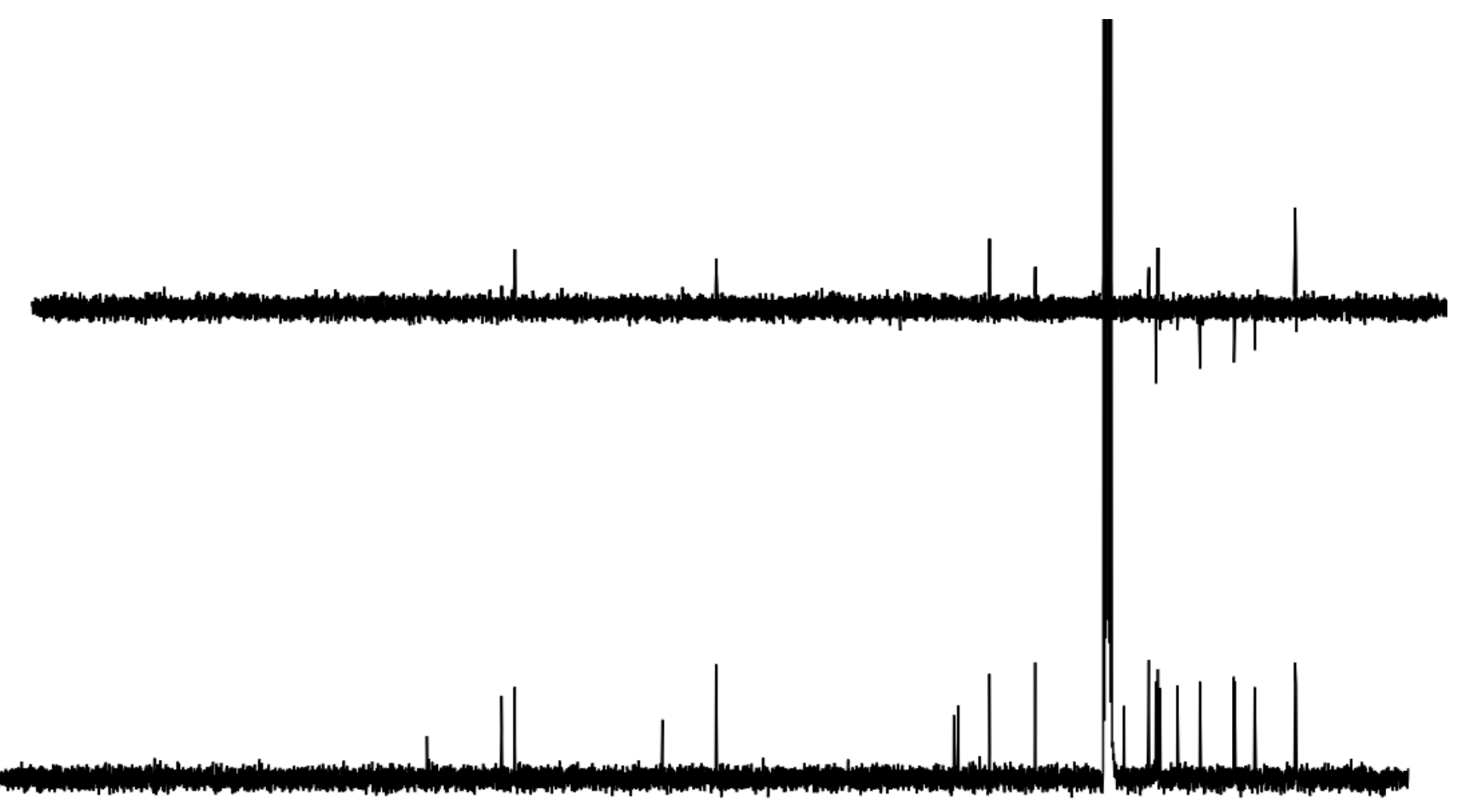

\begin{tabular}{rrrrrrrrrrrrrrrrrrrrrrr}
\hline 230 & 210 & 190 & 170 & 150 & 130 & $\begin{array}{r}110 \\
\mathrm{f1}(\mathrm{ppm})\end{array}$ & 90 & 80 & 70 & 60 & 50 & 40 & 30 & 20 & 10 & 0 & 10
\end{tabular}

Figure S57. The ${ }^{13} \mathrm{C}-\mathrm{NMR}$ and DEPT 135 spectra of 9 (in $\mathrm{CD}_{3} \mathrm{OD}, 400 \mathrm{M}$ 


\section{Identification of Compounds 1-3 in the total ethanol extract of toad eggs}

Compounds 1-3 were identified in total ethanol extract of toad eggs using HPLC-TOF-MS method. The samples were analyzed using a biphenyl column (Kinetex Biphenyl, $5 \mu \mathrm{m}, 4.6 \times 250 \mathrm{~mm}$, Phenomenex, USA) with the mobile phase containing acidified water (0.1\% formic acid) (A) and methanol (B): $50-80 \% \mathrm{~B}$ at 0-30 $\mathrm{min}, 80-91 \% \mathrm{~B}$ at $30-31 \mathrm{~min}, 91-95 \% \mathrm{~B}$ at $31-60,95-100 \% \mathrm{~B}$ at $60-61 \mathrm{~min}$, $100 \%$ B at 61-75 min. The results were showed in Figure S58-S60. 


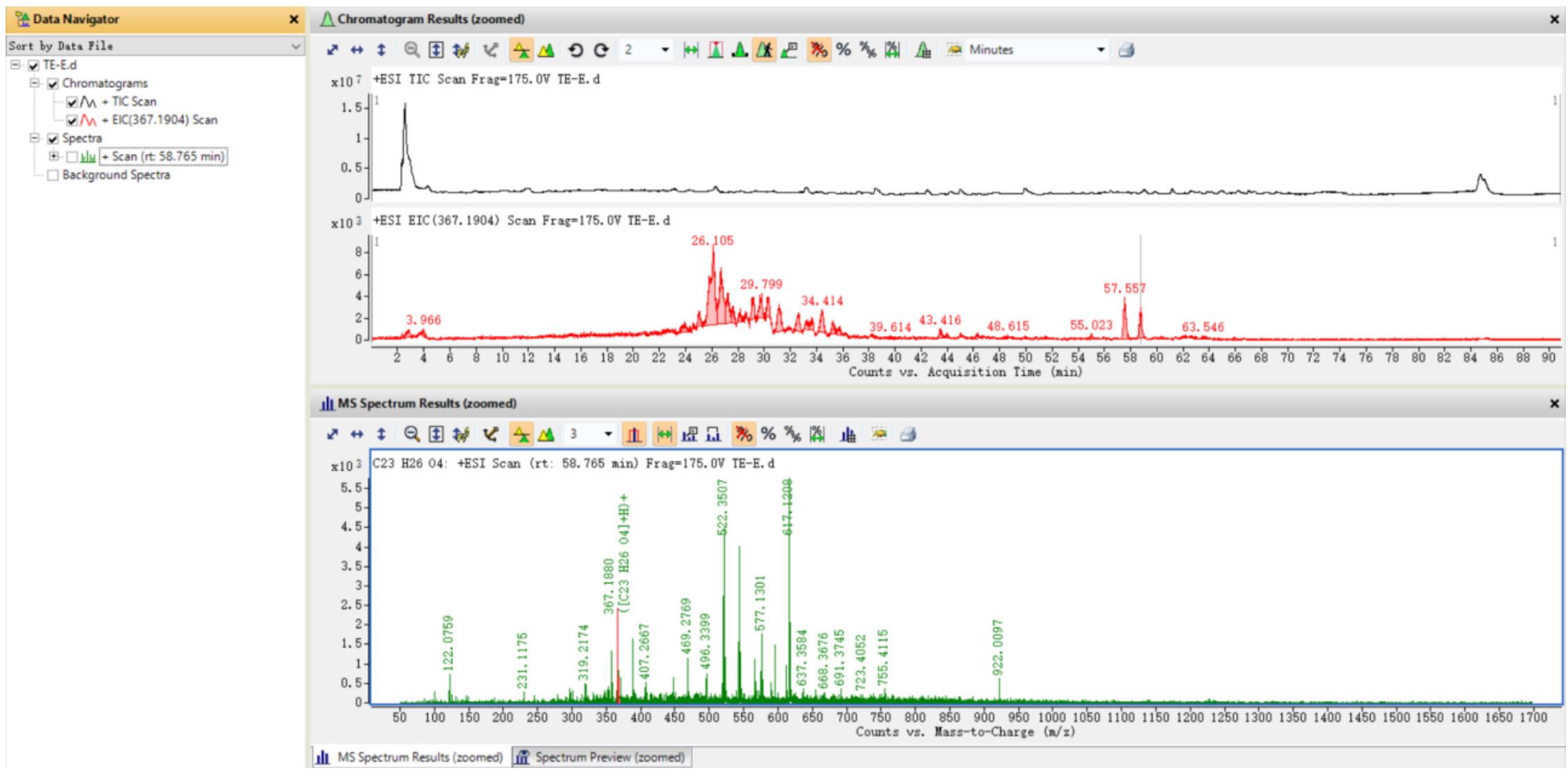

Figure S58. Identification of compound 1 in total ethanol extract of toad eggs using HPLC-TOF-MS [retention time: $58.765 \mathrm{~min}, \mathrm{~m} / z 367.1880$ $[\mathrm{M}+\mathrm{H}]^{+}$(calcd for $\left.\mathrm{C}_{23} \mathrm{H}_{27} \mathrm{O}_{4}, 367.1904\right)$ 


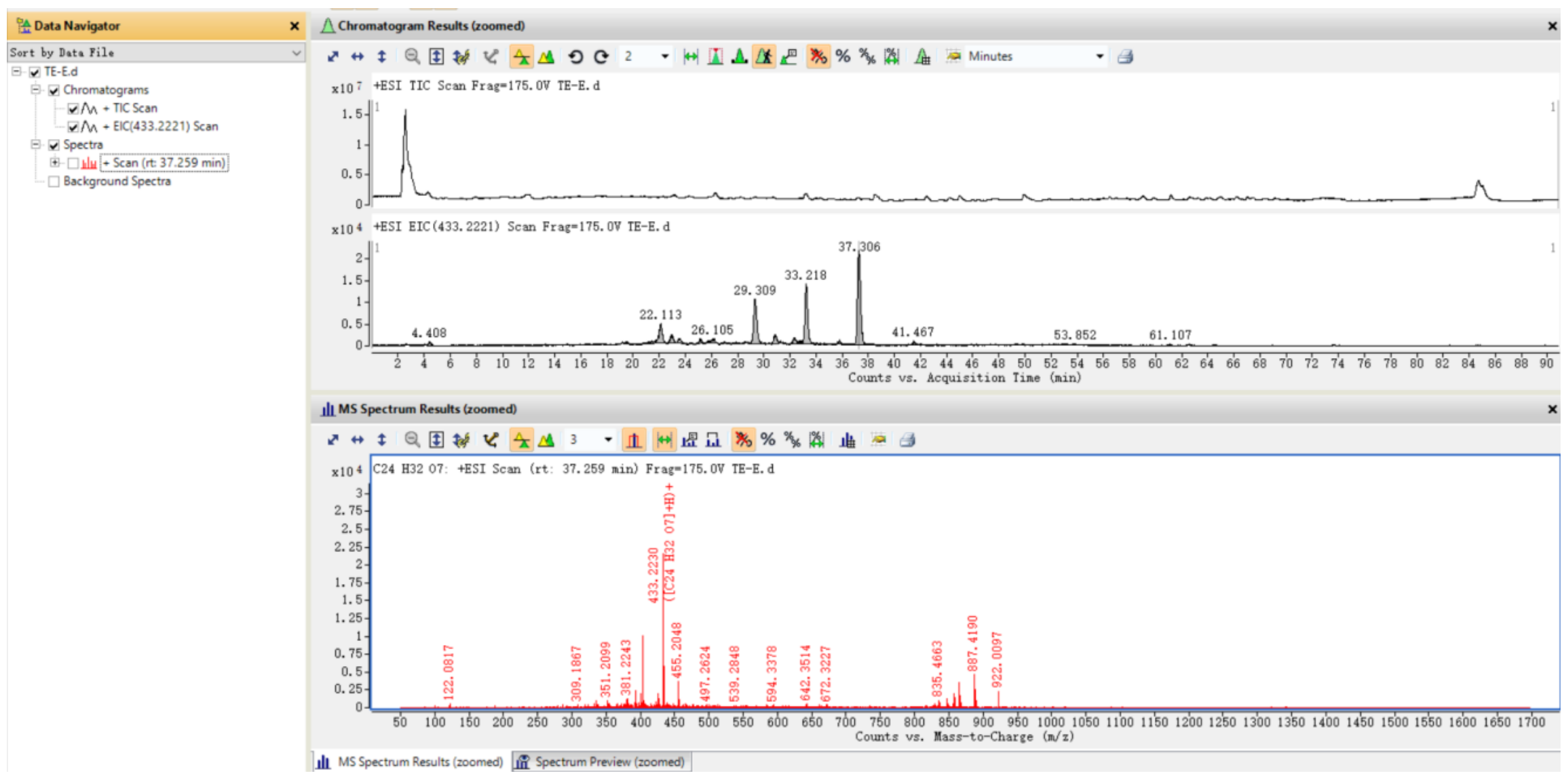

Figure S59. Identification of compound 3 in total ethanol extract of toad eggs using HPLC-TOF-MS [retention time: $37.259 \mathrm{~min}, \mathrm{~m} / \mathrm{z} 433.2230$ $[\mathrm{M}+\mathrm{H}]^{+}$(calcd for $\mathrm{C}_{24} \mathrm{H}_{33} \mathrm{O}_{7}, 433.2221$ ) 


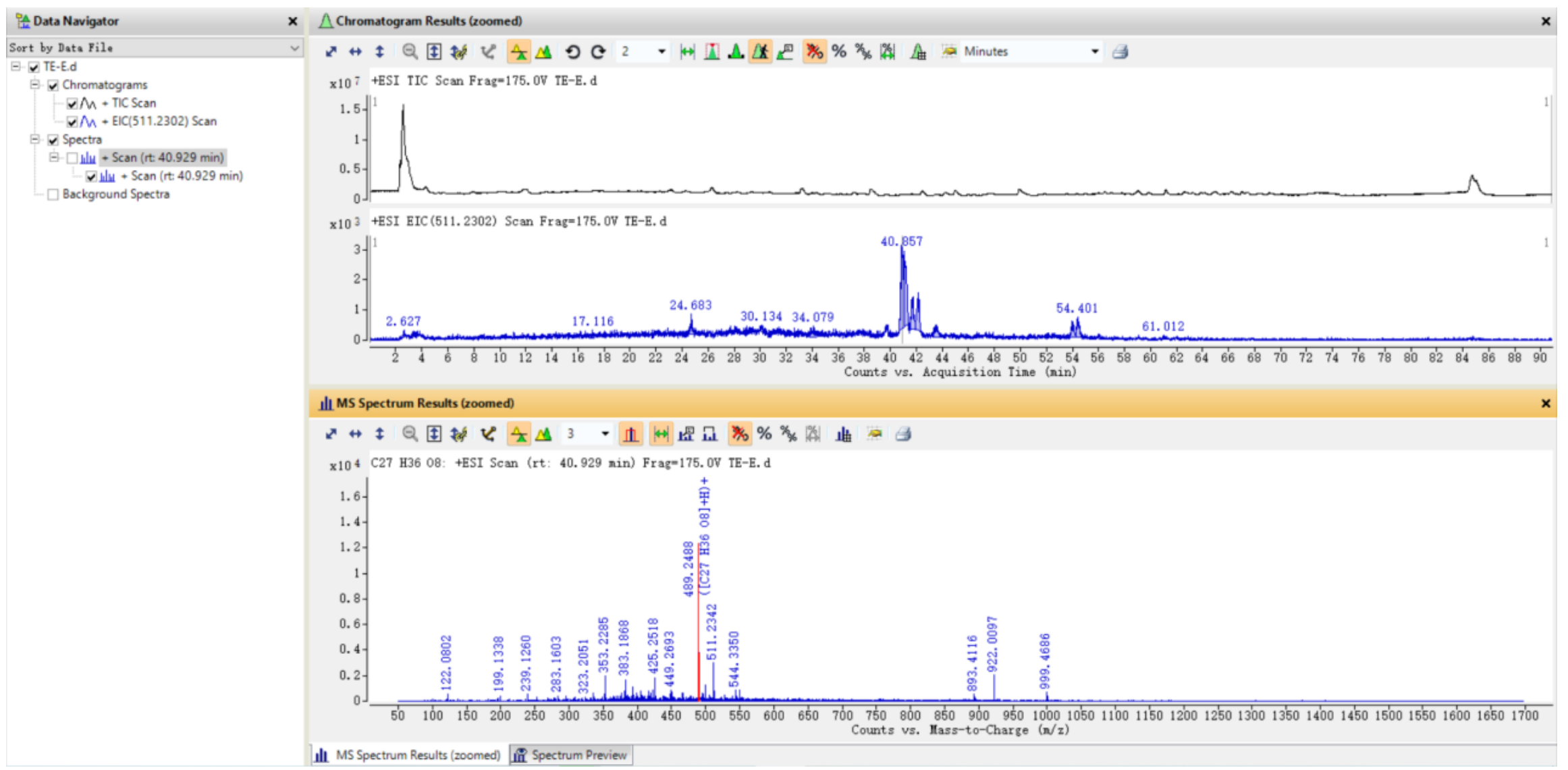

Figure S60. Identification of compound 3 in total ethanol extract of toad eggs using HPLC-TOF-MS [retention time: $40.929 \mathrm{~min}, \mathrm{~m} / \mathrm{z} 489.2488$ $[\mathrm{M}+\mathrm{H}]^{+}$(calcd for $\mathrm{C}_{27} \mathrm{H}_{37} \mathrm{O}_{8}, 489.2483$ ) 


\section{Comparison NMR data of 1 and 2 with those of known compounds.}

Table S7. ${ }^{1} \mathrm{H}$ and ${ }^{13} \mathrm{C}$ NMR data of compound $\mathbf{1}$ and bufogargarizin $\mathrm{C}$.

\begin{tabular}{|c|c|c|c|c|}
\hline \multirow[b]{2}{*}{ position } & \multicolumn{2}{|l|}{$1^{\mathrm{a}}$} & \multicolumn{2}{|c|}{ Bofugarizin $\mathbf{C}^{\mathrm{b}}$} \\
\hline & $\delta_{\mathrm{C}}$, type & $\delta_{\mathrm{H}}{ }^{\mathrm{b}}(J$ in $\mathrm{Hz})$ & $\delta_{\mathrm{C}}$, type & $\delta_{\mathrm{H}}^{\mathrm{b}}(J$ in $\mathrm{Hz})$ \\
\hline $1 \alpha$ & $27.2, \mathrm{CH}_{2}$ & $2.49^{c}$ & $33.3, \mathrm{CH}_{2}$ & $1.79, \mathrm{~m}$ \\
\hline$\beta$ & & $2.49^{\mathrm{c}}$ & & $0.84(t, 11.8)$ \\
\hline $2 \alpha$ & 27.1, $\mathrm{CH}_{2}$ & $2.42, \mathrm{~m}$ & $31.4, \mathrm{CH}_{2}$ & $1.96, \mathrm{~m}$ \\
\hline$\beta$ & & $2.49^{\mathrm{c}}$ & & $1.66, \mathrm{~m}$ \\
\hline 3 & $144.8, \mathrm{CH}$ & $6.70, \mathrm{~m}$ & $69.1, \mathrm{CH}$ & $3.43, \mathrm{~m}$ \\
\hline $4 \alpha$ & $134.1, \mathrm{CH}$ & $6.05, \mathrm{dd}(12.1,1.3)$ & $34.2, \mathrm{CH}_{2}$ & $2.76, \operatorname{dd}(10.2,10.2)$ \\
\hline$\beta$ & & & & $2.51, \mathrm{dd}(10.2,4.4)$ \\
\hline 5 & 192.3, C & & $208.6, \mathrm{C}$ & \\
\hline $6 \alpha$ & $140.5, \mathrm{C}$ & & $63.6, \mathrm{CH}$ & $2.99, \mathrm{dd}(8.6,8.6)$ \\
\hline $7 \alpha$ & $33.2, \mathrm{CH}_{2}$ & $2.19^{\mathrm{c}}$ & $26.0, \mathrm{CH}_{2}$ & $1.66, \mathrm{~m}$ \\
\hline$\beta$ & & $2.73, \mathrm{dd}(14.7,6.7)$ & & $1.54, \mathrm{~m}$ \\
\hline 8 & $51.9, \mathrm{CH}$ & $1.89, \mathrm{~m}$ & $44.8, \mathrm{CH}$ & $1.85, \mathrm{~m}$ \\
\hline 9 & $53.8, \mathrm{CH}$ & $2.57, \mathrm{~m}$ & $52.8, \mathrm{CH}$ & $0.84, \mathrm{~m}$ \\
\hline 10 & $164.2, \mathrm{C}$ & & $79.2, \mathrm{C}$ & \\
\hline $11 \alpha$ & 26.5, $\mathrm{CH}_{2}$ & $1.86, \mathrm{~m}$ & 20.7, $\mathrm{CH}_{2}$ & $1.23, \mathrm{~m}$ \\
\hline$\beta$ & & $1.36, \mathrm{~m}$ & & $1.23, \mathrm{~m}$ \\
\hline $12 \alpha$ & 43.6, $\mathrm{CH}_{2}$ & $1.57, \mathrm{~m}$ & $41.2, \mathrm{CH}_{2}$ & $1.45, \mathrm{~m}$ \\
\hline$\beta$ & & $1.69, \mathrm{dt}(13.8,3.2)$ & & $1.28, \mathrm{~m}$ \\
\hline 13 & $54.9, \mathrm{C}$ & & $49.0, \mathrm{C}$ & \\
\hline 14 & $84.5, \mathrm{C}$ & & $82.9, \mathrm{C}$ & \\
\hline $15 \alpha$ & $33.0, \mathrm{CH}_{2}$ & $2.19^{\mathrm{c}}$ & $31.2, \mathrm{CH}_{2}$ & $1.91, \mathrm{~m}$ \\
\hline$\beta$ & & $1.75, \mathrm{dd}(12.4,8.2)$ & & $1.58, \mathrm{~m}$ \\
\hline $16 \alpha$ & $30.9, \mathrm{CH}_{2}$ & $2.23, \mathrm{~m}$ & $29.9, \mathrm{CH}_{2}$ & $2.05, \mathrm{~m}$ \\
\hline$\beta$ & & $1.83, \mathrm{~m}$ & & $1.62, \mathrm{~m}$ \\
\hline 17 & $51.9, \mathrm{CH}$ & $2.63, \mathrm{dd}(7.2,9.0)$ & $49.5, \mathrm{CH}$ & $2.48, \mathrm{dd}(9.4,6.6)$ \\
\hline 18 & $17.8, \mathrm{CH}_{3}$ & $0.75, \mathrm{~s}$ & $16.9, \mathrm{CH}_{3}$ & $0.61, \mathrm{~s}$ \\
\hline 20 & $124.8, \mathrm{C}$ & & 125.1, C & \\
\hline 21 & $150.4, \mathrm{CH}$ & 7.45, d (2.6) & $150.4, \mathrm{CH}$ & $7.42, \mathrm{~d}(2.5)$ \\
\hline 22 & $149.2, \mathrm{CH}$ & $8.00, \mathrm{dd}(2.6,9.7)$ & $149.4, \mathrm{CH}$ & $7.99, \mathrm{dd}(2.5,9.7)$ \\
\hline 23 & 115.6, CH & $6.30, \mathrm{~d}(9.7)$ & $115.4, \mathrm{CH}$ & $6.27, \mathrm{~d}(9.7)$ \\
\hline 24 & $164.7, \mathrm{C}$ & & $164.8, \mathrm{C}$ & \\
\hline
\end{tabular}


Table S8 ${ }^{1} \mathrm{H}$ and ${ }^{13} \mathrm{C}$ NMR data of compound 2 and hellebrigenin.

\begin{tabular}{|c|c|c|c|c|}
\hline \multirow[b]{2}{*}{ position } & \multicolumn{2}{|r|}{2} & \multicolumn{2}{|c|}{ hellebrigenin } \\
\hline & $\delta_{\mathrm{C}}{ }^{\mathrm{a}}$, type & $\delta_{\mathrm{H}}^{\mathrm{b}}$ & $\delta_{\mathrm{C}}{ }^{\mathrm{a}}$, type & $\delta_{\mathrm{H}}{ }^{\mathrm{b}}$ \\
\hline $1 \alpha$ & $21.4, \mathrm{CH}_{2}$ & 1.82, br d (14.9) & $18.5, \mathrm{CH}_{2}$ & \\
\hline$\beta$ & & $2.38, \mathrm{~m}$ & & \\
\hline $2 \alpha$ & $28.4, \mathrm{CH}_{2}$ & $1.68^{\mathrm{c}}$ & 27.6, $\mathrm{CH}_{2}$ & \\
\hline$\beta$ & & $1.68^{\mathrm{c}}$ & & \\
\hline 3 & $68.1, \mathrm{CH}$ & 4.12, br s & $68.0, \mathrm{C}$ & 4.14, br s \\
\hline $4 \alpha$ & $39.1, \mathrm{CH}_{2}$ & $2.18^{c}$ & $38.6, \mathrm{CH}_{2}$ & \\
\hline$\beta$ & & $1.55^{\mathrm{c}}$ & & \\
\hline 5 & 75.6, C & & 75.8, C & \\
\hline $6 \alpha$ & $37.0, \mathrm{CH}_{2}$ & $1.43^{\mathrm{c}}$ & $37.4, \mathrm{CH}_{2}$ & \\
\hline$\beta$ & & $2.42^{c}$ & & \\
\hline $7 \alpha$ & 24.5, $\mathrm{CH}_{2}$ & $1.29, \mathrm{~m}$ & $25.2, \mathrm{CH}_{2}$ & \\
\hline$\beta$ & & $2.01, \mathrm{~m}$ & & \\
\hline 8 & $41.7, \mathrm{CH}$ & $2.22, \mathrm{~m}$ & $43.0, \mathrm{CH}$ & \\
\hline 9 & $40.1, \mathrm{CH}$ & $1.64^{\mathrm{c}}$ & $33.3, \mathrm{CH}$ & \\
\hline 10 & $55.6, \mathrm{C}$ & & $56.2, \mathrm{CH}$ & \\
\hline $11 \alpha$ & 24.6, $\mathrm{CH}_{2}$ & $1.63^{\mathrm{c}}$ & $23.6, \mathrm{C}$ & \\
\hline$\beta$ & & $1.36^{\mathrm{c}}$ & & \\
\hline $12 \alpha$ & $41.9, \mathrm{CH}_{2}$ & $1.53^{\mathrm{c}}$ & $41.5, \mathrm{CH}$ & \\
\hline$\beta$ & & $1.45^{\mathrm{c}}$ & & \\
\hline 13 & 49.7, C & & $49.4, \mathrm{C}$ & \\
\hline 14 & $86.0, \mathrm{C}$ & & $85.7, \mathrm{C}$ & \\
\hline $15 \alpha$ & $32.7, \mathrm{CH}_{2}$ & $2.05^{\mathrm{c}}$ & $32.3, \mathrm{CH}_{2}$ & \\
\hline$\beta$ & & $1.68^{\mathrm{c}}$ & & \\
\hline $16 \alpha$ & $29.8, \mathrm{CH}_{2}$ & $2.19^{\mathrm{c}}$ & $29.7, \mathrm{CH}_{2}$ & \\
\hline$\beta$ & & $1.75, \mathrm{~m}$ & & \\
\hline 17 & $52.0, \mathrm{CH}$ & $2.54, \mathrm{dd}(9.6,6.5)$ & $52.0, \mathrm{CH}$ & $2.55, \mathrm{dd}(9.3,6.5)$ \\
\hline 18 & $17.3, \mathrm{CH}_{3}$ & $0.72, \mathrm{~s}$ & 17.1, $\mathrm{CH}_{3}$ & \\
\hline 19 & $178.0, \mathrm{C}$ & & $210.1, \mathrm{CH}$ & $10.9, \mathrm{~s}$ \\
\hline 20 & $125.0, \mathrm{C}$ & & $124.9, \mathrm{C}$ & \\
\hline 21 & $150.5, \mathrm{CH}$ & $7.43, \mathrm{~d}(2.5)$ & $150.5, \mathrm{CH}$ & $7.43, \mathrm{~d}(2.5)$ \\
\hline 22 & $149.3, \mathrm{CH}$ & $7.99, \mathrm{dd}(9.7,2.5)$ & $149.3, \mathrm{CH}$ & $7.99, \mathrm{dd}(9.7,2.5)$ \\
\hline 23 & $115.4, \mathrm{CH}$ & $6.27, \mathrm{~d}(9.7)$ & $115.5, \mathrm{CH}$ & $6.28, \mathrm{~d}(9.7)$ \\
\hline 24 & $164.8, \mathrm{C}$ & & 164.7, C & \\
\hline $\begin{array}{l}\text { a } \text { Measu } \\
\text { reported }\end{array}$ & d at $125 \mathrm{M}$ & $\begin{array}{l}{ }^{\mathrm{b}} \text { Measured at } 500 \\
\text { g multiplicity. }\end{array}$ & MHz. ' Ove & pped signals were \\
\hline
\end{tabular}

\section{Proposed biosynthesis pathway of 1}




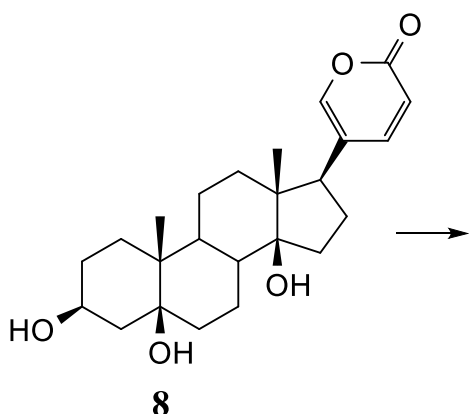

8
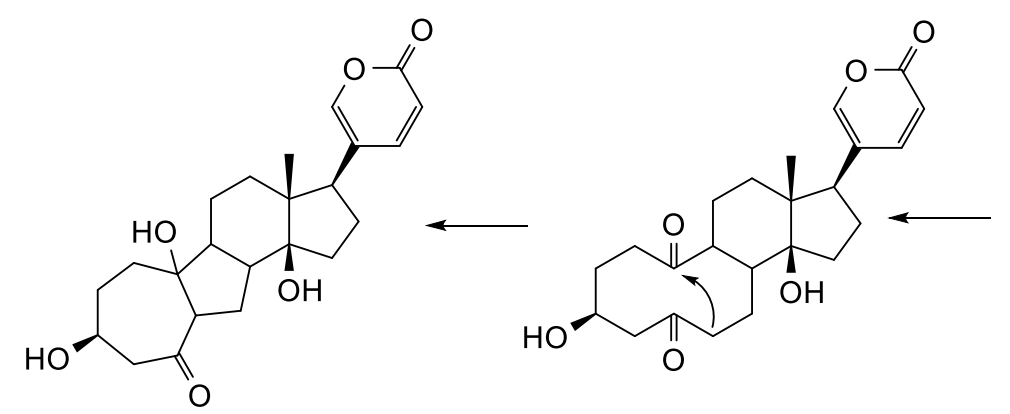

hellebreginol

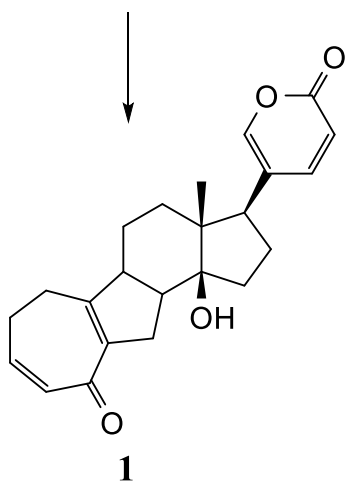

Scheme 1. Proposed biosynthesis pathway of $\mathbf{1}$<smiles>C[C@]12CCC3C(CC[C@@]4(O)C[C@@H](O)CCO[C@]34C)[C@@]1(O)CC[C@@H]2C1C=CC(=O)OC1</smiles>

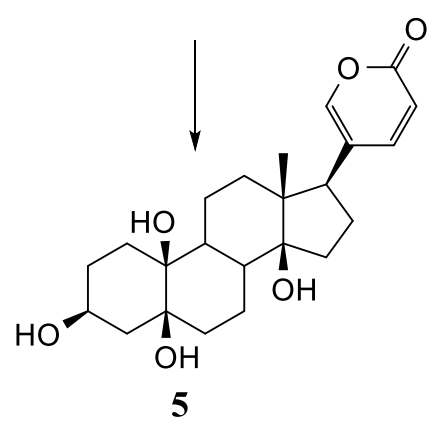

